\title{
Squalus shiraii sp. nov. (Squaliformes, Squalidae), a new species of dogfish shark from Japan with regional nominal species revisited
}

\author{
Sarah T. F. L. Viana ${ }^{1,2}$, Marcelo R. de Carvalho ${ }^{2}$ \\ 1 South African Institute for Aquatic Biodiversity, Private Bag 1015 Grahamstown, 6140, South Africa \\ 2 Departamento de Zoologia, Instituto de Biociências, Universidade de São Paulo. Rua do Matão, Travessa 14, no101, CEP 05508-090, São \\ Paulo, SP, Brazil \\ http://zoobank.org/4A3A5AE9-D263-40A0-8621-430C7822CFF3
}

Corresponding author: Sarah T. F. L. Viana (stviana@gmail.com)

Academic editor: Peter Bartsch • Received 11 March 2020 • Accepted 27 April 2020 • Published 10 June 2020

\begin{abstract}
A new species of deep-water dogfish shark, Squalus shiraii sp. nov., is described herein as endemic to the tropical waters off Southern Japan. This species has been largely misidentified with S. mitsukurii. However, morphological, meristic and morphometric evidence support it to be a separate and undescribed species. Squalus shiraii sp. nov. differs from this species by having body brown in colour dorsally, caudal fin with ventral and dorsal tips markedly tapered and broadly white, dermal denticles uniscuspidate and lanceolate and larger number of precaudal (91-94) and total vertebrae (120-123) (vs. body dark grey to black; caudal fin with ventral and dorsal tips rounded and not white in colour; denticles tricuspidate and rhomboid; 86-90 precaudal and 116-117 total vertebrae). Squalus shiraii sp. nov. is also clearly separated from other Japanese congeners which are herein revisited to include six species, based on the examination of over 150 specimens caught from Japanese waters that were available in ichthyological collections: $S$. mitsukurii, S. japonicus, S. acutirostris, S. brevirostris and S. suckleyi. Squalus mitsukurii, S. japonicus and S. brevirostris are re-described in detail and the neotype of S. japonicus is herein designated. Squalus acutirostris is treated as a valid species with occurrences in Japan, China and Taiwan and, thus, a provisional diagnosis is given, as well as an updated diagnosis of S. suckleyi. A key to Squalus species from the North-western Pacific Ocean is given and main morphological differences between $S$. shiraii sp. nov. and the closest related species are discussed.
\end{abstract}

\section{Key Words}

taxonomy; species diversity; elasmobranch; Squalus; North-western Pacific Ocean

\section{Introduction}

Dogfish sharks of the genus Squalus Linnaeus, 1758 represent commercially-important taxa within the world fish trade, in which individuals are caught through direct or indirect fisheries and traded for consumption of meat, fins and liver oil as primary products. Although highly exploited, the landing reports, observer and logbooks data and/or fisheries surveys have constantly pointed out that accurate identification of species is scarce (e.g. da Silva et al. 2015; Hacohen-Domené et al. 2020). Fisheries management and conservation of dogfish sharks are thus still outstanding as species-specific catch and landing statistics, population threats and trends are not yet acknowledged.

Japan represents one of the world's leading shark fisheries country whose estimated annual shark landing data for between 1992 and 2000 was 19,600-28,700 t (Sonu 1998; Matsunaga et al. 2003). Over 78 elasmobranch species currently evaluated as threatened (VU, vulnerable; CR, critically endangered; EN: endangered) in the Red List of Threatened Species of the International Union for Conservation of Nature (IUCN) are traded in the Japanese shark fin markets, highlighting it as a priority country for elasmobranch conservation (Dulvy et al. 2017), 
even though shark catches have decreased drastically (Sonu 1998). Squalus and other squalid sharks have been exploited in Japan as by-catch fisheries of Pacific salmon gillnets, tuna longliners and squid driftnets (Wilson and Seki 1994; Nakano and Nagasawa 1996; McKinnell and Seki 1998), but fishery statistics related to Squalus stocks are unreported or deficient and usually inserted into the category of "other species".

Five valid species (of 36 valid Squalus species) are often recognised in the country (Chen et al. 1979; Nakaya 1985; Shirai 1989; Nakaya and Shirai 1992; Zhu and Meng 2001; Nakabo 2013; Shinohara et al. 2014; Dyldin 2015): S. blainvillei (Risso, 1827), S. suckleyi (Girard, 1854), S. mitsukurii Jordan \& Snyder, 1903, S. japonicus Ishikawa, 1908 and S. brevirostris Tanaka, 1917. Two additional nominal species, S. acutirostris Zhu, Meng \& Li, 1984 and S. wakiyae Tanaka, 1917, originally described from Japan, are considered junior synonyms of $S$. mitsukurii and $S$. acanthias, respectively (White et al. 2007c; White and Last 2013). A sixth species, S. formosus White \& Iglésias, 2011, a Taiwanese endemic, is likely to inhabit Japanese waters as well, but requires confirmation. These species are found on lower continental shelves, upper continental slopes and, more rarely, on insular slopes and seamounts of the North Pacific and Eastern Indian Oceans between Russia, Japan, China, South Korea, Taiwan and the Philippines. These species are all now classified as IUCN Data Deficient (DD), except for S. suckleyi (Least Concern, LC) (IUCN 2020). A key criterion to be inserted into the first category is unreliability of species identification and, thus, species-specific data are inaccurate or doubtful and may not be incorporated into the assessments.

Systematic approaches of the last decade support that S. mitsukurii comprises a species complex that also includes $S$. blainvillei, a species originally described from the Mediterranean Sea (Ward et al. 2007; Naylor et al. 2012; Veríssimo et al. 2017). Distribution records of $S$. mitsukurii from outside the North Pacific Ocean were successively refuted: Duffy and Last (2007b) and Last et al. (2007d) for the South Pacific Ocean; Viana et al. $(2016,2017 b, 2018)$ for the South Atlantic and Western Indian Oceans; and Verissimo et al. (2017) for the North-eastern Atlantic and Pfleger et al. (2018) for the North-western Atlantic Oceans. Additional species, previously misidentified with $S$. mitsukurii, were also more recently recognised in the North Pacific Ocean, S. hawaiiensis Daly-Engel, Koch, Anderson, Cotton \& Grubbs, 2018 and S. boretzi Dolganov, 2019. Records of $S$. japonicus and $S$. brevirostris are exclusive to the Indo-Pacific region (Chen et al. 1979; Compagno 2000; Last et al. 2007b; Manjaji-Matsumoto 2017) with uncertainties regarding their occurrences in Northern Australia and Indonesia due to morphological similarities with S. megalops (MacLeay, 1881) and S. nasutus Last, Marshall \& Stevens, 2007.

Taxonomic confusions within this Linnaean group are ordinary because of fair original descriptions, in- distinct morphological diagnostic characters and exaggerated availability of synonyms. Species delimitation using DNA barcoding of mitochondrial genes alone has shown to be ineffective on the genus (e.g. Bineesh et al. 2016; Vella et al. 2017) when non-designation of species identifications in the DNA reference libraries persists as collection-based data are not incorporated within the taxonomic investigations. Comparative examination of over 150 specimens of Squalus from the North Pacific Ocean, particularly from Japan, support the recognition of a novel species that has not been included in the latest molecular genetic and morphological taxonomic analyses of the genus. The present study aimed to describe the new species, based on morphological characters and to revise the nominal species of Squalus occurring in the region.

\section{Material and methods}

\section{Morphological analysis and species description}

Comparative analyses of external morphology were made, based on specimens preserved in $70 \%$ ethanol. Maturity stage was determined according to Compagno (1988). Description of external morphology and colouration is in accordance with Last et al. (2007f). Colouration is described from preserved specimens unless otherwise noted. Other terminology follows Cappetta (1987) for dentition and Deynat and Séret (1996) for dermal denticles. Synonyms for species indicate authorship, date and page when possible. Tooth samples were taken from upper and lower jaws (three lateral teeth towards the first series) and later examined using a stereoscopic microscope. Squamation is based on skin samples measuring $1 \mathrm{~cm}^{2}$ obtained from below the first dorsal fin (right side) and was analysed under a Scanning Electron Microscope (SEM) at the Instituto de Biociências, Universidade de São Paulo (IBUSP) and Rhodes University (RU).

\section{Morphometric analysis}

External measurements were obtained from preserved specimens using a digital caliper with $0.1 \mathrm{~mm}$ precision and/or a metric tape for measurements larger than 150 mm. Morphometrics follow Last et al. (2007f) and Viana et al. (2016) and are expressed as percentages of total length ( $\%$ TL). Morphometrics of the species are provided in Tables 1 to 5 . Single values in the diagnosis and description correspond to holotype and range values to all other material from which data was obtained, except when first mentioned. Values for mean and standard deviation in tables include all specimens for which data were taken. Range values for diagnosis, discussion and identification key include adult specimens only, unless otherwise stated in text. 


\section{Meristic data}

Tooth counts were taken according to Herman et al. (1989) from preserved specimens. Vertebral counts were obtained through film and digital radiographs and follows Springer and Garrick (1964). Meristic data of the species are provided in Table 6 . Single values in the diagnosis and description correspond to holotype and range values to additional material from which data was obtained except otherwise noted.

\section{Species illustration}

A digital camera was utilised for photographing of specimens in dorsal, ventral and lateral views, as well as particular body parts (e.g. fins, claspers). Teeth were photographed using a digital camera attached to a stereoscope microscope Leica DFC295.

\section{Species distribution}

QGIS 2.14.2 Essen (QGIS Development Team, QGIS Geographic Information System, Open Source Geospatial Foundation Project; http://qgis.osgeo.org) and Google Earth were run to create maps of geographical distribution. Coordinates of each specimen examined were obtained from collecting event data available in the ichthyological databases. For specimens without accurate coordinates, the nearest locality data was considered for plotting maps.

\section{Comparative material}

Comparative material is listed under each species account when applicable. Data were also taken from Baranes (2003), Last et al. (2007a,b,c,d,g), White et al. (2007a,b), Ebert et al. (2010), White and Iglésias (2011), Viana and Carvalho (2016, 2018a,b), Viana et al. (2016, 2017a,b), Daly-Engel et al. (2018) and Dolganov (2019). Vertebral counts are summarised in Suppl. material 1: Table S1.

\section{Abbreviations}

Institutional acronyms follow Sabaj (2016).

\section{Results}

\section{Squalus shiraii sp. nov.}

http://zoobank.org/68278ED1-AADE-40A2-8155-A3D2AF7B6894

Figs 1, 3A-B, 7A-B, 8A; Tables 1, 6

Holotype. HUMZ 149389, adult male, $590 \mathrm{~mm}$ TL, Okinawa Trough, $25^{\circ} 37^{\prime} 28^{\prime \prime N}, 126^{\circ} 05^{\prime} 35^{\prime \prime} \mathrm{E}$ to $25^{\circ} 38^{\prime} 12^{\prime \prime N} 126^{\circ} 07^{\prime} 83^{\prime \prime E}$. Collected on 2 August 1994 by unknown collector.

Paratypes. HUMZ 80329, adult female, $770 \mathrm{~mm}$ TL, East China Sea, $25^{\circ} 33.8^{\prime} \mathrm{N}, 126^{\circ} 25.2^{\prime} \mathrm{E}, 310 \mathrm{~m}$ depth, longline, collected on 4 December 1978 by T. Kanayama; HUMZ 80330, juvenile male, $365 \mathrm{~mm}$ TL, locality and collection data same as HUMZ 80329; HUMZ 80331, adult female, $755 \mathrm{~mm}$ TL, locality and collection data same as HUMZ 80329; HUMZ 101718, adult male, $685 \mathrm{~mm} \mathrm{TL}$, off north-west of Okinawa-jima Island, Japan, collected in July 1983 by K. Nakaya; HUMZ 146165, adult male, 600 $\mathrm{mm} \mathrm{TL}, 31^{\circ} 58.5^{\prime} \mathrm{N}, 173^{\circ} 09.5^{\prime} \mathrm{E}, 390 \mathrm{~m}$ depth, longline, collected on 21 February 1997; HUMZ 149391, adult male, $623 \mathrm{~mm}$ TL, locality and collection data same as holotype; HUMZ 149392, adult male, 595 mm TL, locality and collection data same as holotype; HUMZ 149394, adult male, $623 \mathrm{~mm}$ TL, locality and collection data same as holotype; HUMZ 149395, adult male, $595 \mathrm{~mm}$ TL, locality and collection data same as holotype; HUMZ 149426, adult male, $630 \mathrm{~mm}$ TL, locality and collection data same as holotype; HUMZ 149434, adult male, 580 mm TL, Okinawa Trough, $25^{\circ} 40^{\prime} 32^{\prime \prime N}, 126^{\circ} 14^{\prime} 18^{\prime \prime E}$ to $25^{\circ} 41^{\prime} 43^{\prime \prime} \mathrm{N}, 126^{\circ} 16^{\prime} 08^{\prime \prime} \mathrm{E}$, collected on 2 August 1994 by unknown collector; HUMZ 149438, adult male, $605 \mathrm{~mm}$ TL, locality and collection data same as HUMZ 149434.

Type locality. Okinawa Trough, Japan, $25^{\circ} 37^{\prime} 28^{\prime \prime} \mathrm{N}$, $126^{\circ} 05^{\prime} 35^{\prime \prime} \mathrm{E}$ to $25^{\circ} 38^{\prime} 12^{\prime \prime} \mathrm{N}, 126^{\circ} 07^{\prime} 83^{\prime \prime E}, 2$ August 1994, unknown collector.

Additional material. HUMZ 495, juvenile female, 350 mm TL, unknown locality; HUMZ 39991, juvenile male, $260 \mathrm{~mm}$ TL, unknown locality.

Diagnosis. Single values correspond to the holotype and range to paratypes, respectively. A Squalus species that is separated from its regional congeners by: first dorsal fin conspicuously upright (vs. first dorsal fin prone); pectoral-fin posterior margin falcate (vs. not falcate); upper and lower caudal lobes markedly slender with dorsal and ventral caudal tips pointed and broadly white (vs. upper and lower caudal lobes wide with dorsal and ventral caudal tips rounded and greyish in colour); dermal denticles lanceolate, except for $S$. brevirostris (vs. tricuspidate); smaller claspers with clasper outer length $3.3 \%, 3.3 \%-3.4 \%$ TL and clasper inner length $6.3 \%, 5.9 \%-6.3 \%$ TL (vs. $4.5 \%-4.9 \%$ TL, $6.9 \%-8.4 \%$ TL for $S$. mitsukurii vs. $4.8 \%$, 4.3\%-5.6\% TL and 7.5\%, 7.2\%-9.1\% TL for S. japonicus; vs. $3.9 \%, 3.9 \%-5.1 \%$ TL and $7.1 \%, 7.0 \%-8.1 \%$ TL for $S$. brevirostris). Squalus shiraii sp. nov. further differentiates from $S$. mitsukurii by more elongate eyes, its length $4.9 \%$, $4.4 \%-4.9 \%$ TL (vs. 3.6\%, 3.1\%-4.0\% TL) and smaller fifth gill slit, its length $1.7 \%, 1.7 \%-2.1 \%$ TL (vs. $2.3 \%$, $2.2 \%-2.6 \% \mathrm{TL}$ ). It is separated from $S$. japonicus and $S$. brevirostris by prenarial length 5.5\%, 5.4\%-5.6\% TL (vs. $5.9 \%, 5.9 \%-6.8 \%$ TL for $S$. japonicus vs. $4.0 \%, 3.8 \%$ $4.3 \%$ TL for $S$. brevirostris) and width of first dorsal-fin 

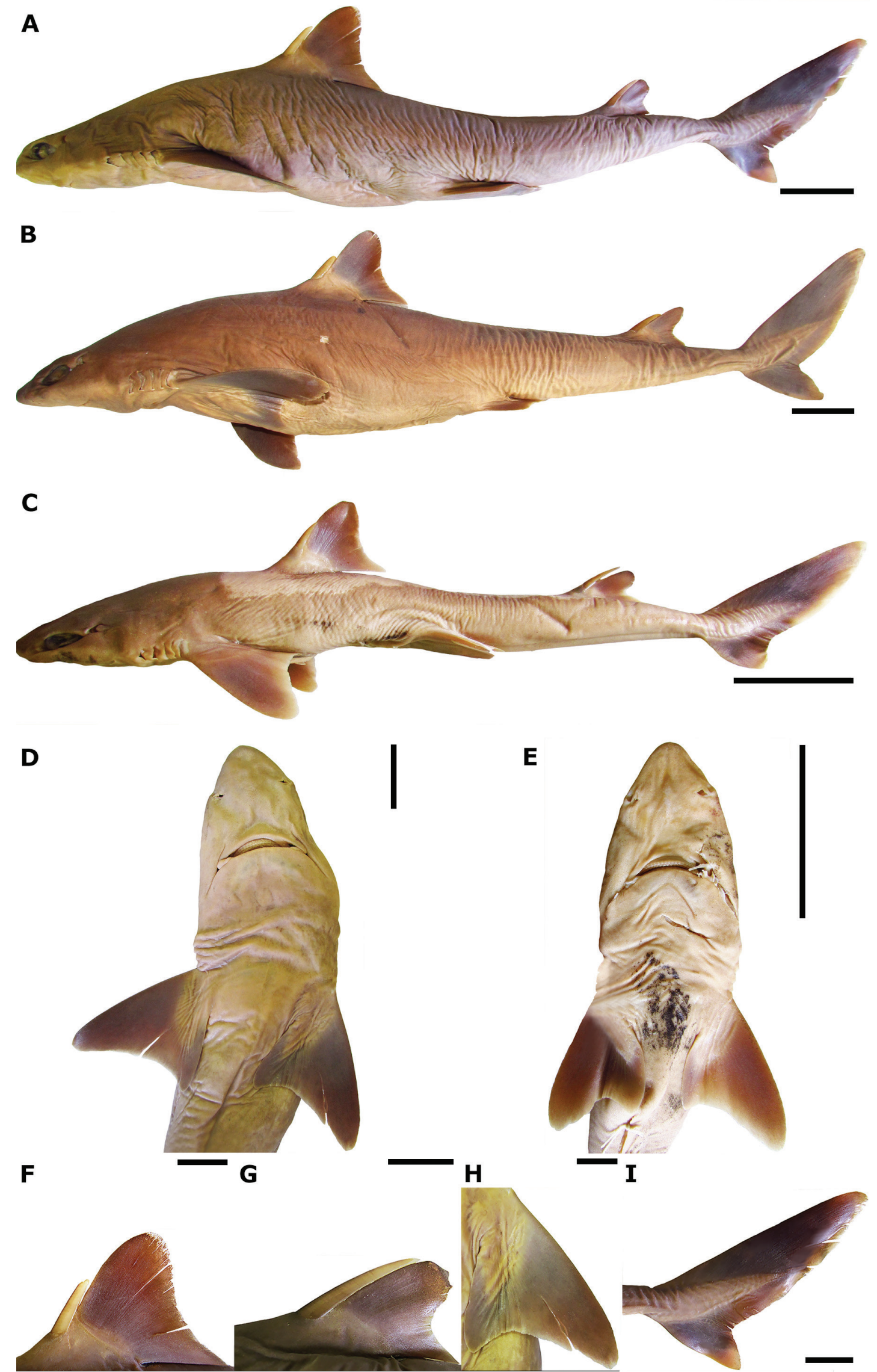

Figure 1. Squalus shiraii sp. nov.: lateral (A-C) and ventral (D, E) views; first (F) and second (G) dorsal fins; pectoral (H) and caudal (I) fins. HUMZ 149389 (holotype), adult male, $590 \mathrm{~mm}$ TL (A, D, F-I); HUMZ 80329 (paratype), adult female, $770 \mathrm{~mm}$ TL (B); HUMZ 80330 (paratype), juvenile male, $365 \mathrm{~mm}$ TL (C, E). Scale bars: $50 \mathrm{~mm}$ (A-E); $20 \mathrm{~mm}$ (F-I). 
spine $0.9 \%, 0.9 \%-0.9 \%$ TL (vs. $0.7 \%, 0.5 \%-0.8 \%$ TL for S. japonicus vs. $0.7 \%, 0.5 \%-0.7 \%$ TL for S. brevirostris). Squalus shiraii sp. nov. further differs from $S$. japonicus by shorter snout, its preorbital length $7.9 \%, 7.4 \%-7.9 \%$ TL (vs. 8.7\%, 8.7\%-9.3\% TL for S. japonicus), smaller preoral length $10.4 \%, 9.4 \%-10.4 \%$ TL (vs. $10.7 \%$, $10.7 \%-12.2 \% \mathrm{TL})$, larger first dorsal fin, its base length $7.7 \%, 7.7 \%-8.7 \%$ TL and first dorsal-fin posterior margin length $9.2 \%, 9.0 \%-9.3 \%$ TL (vs. $6.7 \%, 6.6 \%-7.5 \%$ TL and $7.8 \%, 6.7 \%-8.6 \%$ TL for $S$. japonicus) and wider pectoral fin, its posterior margin length $10.0 \%, 10.0 \%-$ $11.7 \%$ TL (vs. $8.6 \%, 7.8 \%-9.2 \%$ TL). It is separated from S. brevirostris by wider internarial space, its width $4.8 \%$, $4.1 \%-4.8 \%$ TL (vs. 3.6\%, 3.4\%-3.8\% TL for S. brevirostris), smaller second dorsal fin with inner margin length $3.6 \%, 3.2 \%-4.1 \%$ TL (vs. $5.0 \%, 4.8 \%-5.7 \%$ TL), shorter pectoral-fin inner margin, its length $7.3 \%, 7.3 \%-8.3 \%$ TL (vs. 10.3\%, 9.8\%-11.2\% TL) and narrower caudal fin, its width at caudal fork $6.4 \%, 5.7 \%-6.4 \%$ TL (vs. $6.6 \%$, $6.6 \%-7.3 \%$ TL). Squalus shiraii is distinguished from $S$. formosus regardless of maturity by having more elongate precaudal $(78.3 \%, 78.6 \%-80.5 \% \mathrm{TL})$, pre-second $(60.5 \%$, $61.1 \%-63.4 \%$ TL) and pre-first $(30.5 \%, 29.6 \%-31.2 \%$ TL) dorsal length (vs. 77.6\%-78.3\% TL, 59.4\%-59.7\% TL and $28.1 \%-29.0 \%$ in S. formosus).

Description. Single values correspond to the holotype and range to paratypes, respectively.

External morphology. Body elongate (590-770 mm maximum TL in adults), fusiform and robust, arched dorsally from anterior margin of the eye to insertion of first dorsal fin, turning straight from abdomen to caudal fin (Fig. 1A-C); body equally deep from head to abdomen with head height $0.9(0.9-1.0)$ times trunk height and 0.9 (0.8-1.0) times abdomen height; body with its greatest width at head (head width $1.1(1.0-1.2)$ times trunk width and 1.3 (1.2-1.5) times abdomen width. Head flattened anterior-dorsally, arched posterior-dorsally and elongate, its length $24.1 \%(21.9 \%-23.9 \%)$ TL. Snout conspicuously obtuse at tip and large (preorbital length 7.9\%, 7.4\%8.1\% TL) (Fig. 1D-E); anterior margin of nostrils strongly broad and bi-lobed, its distance to snout tip 1.1 (1.1-1.3) times its distance to upper labial furrow; prenarial length 0.5 (0.5-0.6) times preoral length; internarial space 1.0 (0.8-1.1) times eye length. Eyes oval and enlarged, its length 3.1 (1.9-3.4) times eye height; eyes with anterior margin convex and posterior margin slightly notched. Prespiracular length 1.7 (1.7-1.7) times preorbital length and $0.6(0.6-0.6)$ times prepectoral length. Spiracles crescent and large, its length $1.5 \%(1.0 \%-1.3 \%) \mathrm{TL}$, located laterally behind the eyes. Gill slits tall and somewhat vertical, placed anteriorly to pectoral-fin origin; fifth gill slit height 1.4 (1.1-1.7) times first gill slit height.

Preoral length $1.3(1.3-1.5)$ times mouth width and 0.4 (0.4-0.5) times head length. Upper labial furrow short (its length $0.5,0.4-0.5$ times eye length) with thick and small fold; lower labial furrow elongate with inconspicuous fold. Mouth arched and broad, its width 1.5 (1.3-1.4) times prenarial length and 1.7 (1.5-1.7) times internarial space. Teeth similar in both jaws; upper teeth smaller than lower teeth; teeth with cusp oblique, thick and small; mesial cutting edge slightly convex; distal heel rounded; mesial heel notched; apron short and heavy (Fig. 3A, B). Two and two to three series of functional teeth in the upper and lower jaws, respectively, 13-14 for holotype (13-14 for paratypes) teeth rows on upper jaw and 11-11 (11-12) teeth rows on lower jaw.

Origin of first dorsal fin over the vertical line traced at pectoral-fin insertion. First dorsal fin elongate, its length 1.5 (1.4-1.7) times height of first dorsal fin; first dorsal fin conspicuously tall, its height 1.7 (1.5-1.8) times first dorsal-fin inner margin length; first dorsal-fin anterior margin convex, posterior margin straight in the upper half and conspicuously concave in the lower half; first dorsal-fin apex rounded and evidently slender at the fin web; first dorsal-fin free rear tip triangular and first dorsal-fin inner margin short, its length $0.6(0.5-0.7)$ times first dorsal-fin base length (Fig. 1F, J). First dorsal-fin spine thick with base width $0.9 \%(0.9-0.9 \%)$ TL and elongate (first dorsal-fin spine length $0.5,0.4-0.5$ times height of first dorsal fin), although never reaching first dorsal-fin apex. Interdorsal space $1.0(1.0-1.2)$ times prepectoral length and 2.3 (2.2-2.8) times dorsal-caudal space. Pre-second dorsal length 2.0 (2.0-2.1) times pre-first dorsal length. Second dorsal fin elongate, its length $0.9(0.7-0.9)$ times length of first dorsal fin; second dorsal fin tall, its height 1.6 (1.4-1.8) times second dorsal-fin inner margin length; second dorsal-fin anterior margin convex; second dorsal-fin posterior margin strongly concave and falcate; second dorsal-fin apex rounded and lobe-like; second dorsal-fin inner margin small, its length 0.5 (0.5-0.6) times second dorsal-fin base length (Fig. $1 \mathrm{G}, \mathrm{K}$ ). Second dorsal-fin spine thick with base width $0.7 \%(0.7 \%-0.8 \%)$ TL; second dorsal-fin spine elongate, its length $0.9(0.7-$ $0.9)$ times height of second dorsal fin and 1.3 (1.2-1.6) times length of first dorsal-fin spine, although not reaching second dorsal-fin apex.

Prepectoral length $3.0(2.8-2.9)$ times preorbital length. Pectoral fins with anterior and inner margins conspicuously convex; pectoral-fin posterior margin markedly concave; pectoral-fin apex and free rear tips rounded and lobe-like; pectoral-fin apex conspicuously transcending the horizontal line traced at pectoral-fin free rear tip (Fig. 1H, L); pectoral fins markedly falcate and broad with pectoral-fin posterior margin $1.0(0.4-$ 1.0) times trunk height; pectoral-fin anterior margin 2.0 (1.7-2.0) times pectoral-fin inner margin length and 1.4 (1.4-3.2) times pectoral-fin posterior margin length. Prepelvic length 1.8 (2.0-2.2) times prepectoral length. Pelvic fins located slightly nearer to the first dorsal fin than the second dorsal fin. Pelvic fins with margins straight and apex rounded; pelvic-fin free rear tips rounded to slightly pointed, thin and lobe-like. Adult males with clasper somewhat transcending the pelvic fin; clasper inner length $1.2(0.3-1.2)$ times pelvic-fin inner margin length; clasper groove medial-dorsally and vertical, pro- 
Table 1. External measurements of S. shiraii sp. nov. expressed as percentage of total length (\% TL). Values for the holotype and a paratype (CSIRO H6292-10) of S. formosus are also given for comparisons. TL is expressed in millimeters. H: holotype; N: number of specimens; $x$ : mean; SD: standard deviation.

\begin{tabular}{|c|c|c|c|c|c|c|c|c|c|}
\hline & \multicolumn{5}{|c|}{ Squalus shiraii sp. nov. } & \multicolumn{4}{|c|}{ S. formosus } \\
\hline & $\mathbf{H}$ & $\mathbf{N}$ & Paratypes & $x$ & SD & $\mathbf{H}$ & Paratype & $x$ & SD \\
\hline Total length (mm) & 590.0 & 3 & $365.0-770.0$ & 602.5 & 174.6 & 720.0 & 335.0 & 527.5 & 272.2 \\
\hline Precaudal length & 78.3 & 3 & $78.6-80.5$ & 79.3 & 1.0 & 78.3 & 77.6 & 78.0 & 0.5 \\
\hline Pre-second dorsal length & 60.5 & 3 & $61.1-63.4$ & 61.7 & 1.2 & 59.7 & 59.4 & 59.6 & 0.2 \\
\hline Pre-first dorsal length & 30.5 & 3 & $29.6-31.2$ & 30.3 & 0.7 & 28.1 & 29.0 & 28.5 & 0.6 \\
\hline Pre-vent length & 47.3 & 3 & $46.8-48.7$ & 47.5 & 0.8 & 47.6 & 46.6 & 47.1 & 0.8 \\
\hline Prepelvic length & 44.1 & 3 & $44.5-46.8$ & 45.1 & 1.2 & 43.1 & 44.8 & 43.9 & 1.2 \\
\hline Prepectoral length & 23.8 & 3 & $21.6-23.1$ & 22.6 & 1.1 & 21.9 & 20.4 & 21.2 & 1.1 \\
\hline Head length & 24.1 & 3 & 21.9-23.9 & 23.3 & 1.0 & 23.9 & 21.9 & 22.9 & 1.4 \\
\hline Prebranchial length & 20.3 & 3 & $18.2-20.4$ & 19.5 & 1.0 & 18.6 & 12.8 & 15.7 & 4.1 \\
\hline Prespiracular length & 13.4 & 3 & $12.2-14.1$ & 13.1 & 0.8 & 12.0 & 12.7 & 12.3 & 0.5 \\
\hline Preorbital length & 7.9 & 3 & $7.4-8.1$ & 7.7 & 0.3 & 7.0 & 7.4 & 7.2 & 0.3 \\
\hline Prenarial length & 5.5 & 3 & $5.4-5.6$ & 5.5 & 0.1 & 4.5 & 4.9 & 4.7 & 0.3 \\
\hline Preoral length & 10.4 & 3 & $9.4-10.8$ & 10.0 & 0.7 & 8.7 & 9.6 & 9.2 & 0.6 \\
\hline Inner nostril-labial furrow space & 4.9 & 3 & $4.3-5.0$ & 4.7 & 0.4 & 4.2 & 4.7 & 4.5 & 0.3 \\
\hline Mouth width & 8.0 & 3 & $7.2-7.4$ & 7.4 & 0.4 & 7.7 & 7.8 & 7.8 & 0.1 \\
\hline Labial furrow length & 2.4 & 3 & $1.8-2.3$ & 2.2 & 0.3 & 2.3 & 2.3 & 2.3 & 0.0 \\
\hline Internarial space & 4.8 & 3 & $4.1-4.8$ & 4.6 & 0.3 & 4.1 & 4.3 & 4.2 & 0.2 \\
\hline Interorbital space & 8.4 & 3 & $7.6-8.5$ & 8.2 & 0.4 & 8.0 & 8.8 & 8.4 & 0.6 \\
\hline Eye length & 4.9 & 3 & $4.4-5.8$ & 4.9 & 0.6 & 4.6 & 5.2 & 4.9 & 0.5 \\
\hline Eye height & 1.6 & 3 & $1.7-2.4$ & 1.9 & 0.3 & 1.8 & 2.3 & 2.0 & 0.3 \\
\hline Spiracle length & 1.5 & 3 & $1.0-1.3$ & 1.3 & 0.2 & 1.5 & 1.9 & 1.7 & 0.2 \\
\hline First gill-slit height & 1.2 & 3 & $1.2-1.7$ & 1.3 & 0.3 & 2.0 & 1.9 & 2.0 & 0.1 \\
\hline Fifth gill-slit height & 1.7 & 3 & $1.9-2.1$ & 1.9 & 0.2 & 2.3 & 2.3 & 2.3 & 0.0 \\
\hline Interdorsal space & 23.8 & 3 & $24.0-25.7$ & 24.6 & 0.9 & 25.1 & 24.3 & 24.7 & 0.6 \\
\hline Dorsal-caudal space & 10.3 & 3 & $9.1-10.9$ & 10.1 & 0.8 & 10.1 & 10.5 & 10.3 & 0.3 \\
\hline Pectoral-pelvic space & 19.6 & 3 & $17.1-22.9$ & 19.9 & 2.4 & 21.3 & 18.9 & 20.1 & 1.7 \\
\hline Pelvic-caudal space & 27.3 & 3 & $25.5-26.7$ & 26.5 & 0.8 & 27.4 & 26.4 & 26.9 & 0.7 \\
\hline First dorsal-fin length & 12.6 & 3 & $12.7-13.4$ & 13.0 & 0.4 & 14.2 & 13.0 & 13.6 & 0.9 \\
\hline First dorsal-fin anterior margin & 11.3 & 3 & $10.6-11.7$ & 11.3 & 0.5 & 12.9 & 12.9 & 12.9 & 0.0 \\
\hline First dorsal-fin base length & 7.7 & 3 & $7.0-8.7$ & 7.9 & 0.7 & 8.1 & 7.4 & 7.8 & 0.5 \\
\hline First dorsal-fin height & 8.1 & 3 & $7.9-8.8$ & 8.3 & 0.4 & 10.0 & 9.0 & 9.5 & 0.7 \\
\hline First dorsal-fin inner margin & 4.8 & 3 & $4.7-5.4$ & 5.0 & 0.3 & 6.0 & 6.0 & 6.0 & 0.0 \\
\hline First dorsal-fin posterior margin & 9.2 & 3 & $8.4-9.3$ & 9.0 & 0.4 & 10.2 & 9.5 & 9.8 & 0.5 \\
\hline First dorsal-fin spine length & 3.9 & 3 & $3.3-4.3$ & 3.8 & 0.4 & 6.3 & 3.4 & 4.8 & 2.1 \\
\hline First dorsal-fin spine base width & 0.9 & 3 & $0.9-0.9$ & 0.9 & 0.0 & 1.1 & 0.9 & 1.0 & 0.2 \\
\hline Second dorsal-fin length & 11.3 & 3 & $9.9-11.9$ & 11.2 & 0.9 & 12.8 & 13.6 & 13.2 & 0.6 \\
\hline Second dorsal-fin anterior margin & 10.7 & 3 & $9.7-11.3$ & 10.5 & 0.7 & 11.9 & 12.6 & 12.3 & 0.5 \\
\hline Second dorsal-fin base length & 7.8 & 3 & $6.7-7.7$ & 7.3 & 0.5 & 7.7 & 8.3 & 8.0 & 0.4 \\
\hline Second dorsal-fin height & 5.6 & 3 & $5.7-6.6$ & 6.0 & 0.5 & 6.9 & 7.0 & 6.9 & 0.1 \\
\hline Second dorsal-fin inner margin & 3.6 & 3 & $3.2-4.7$ & 3.9 & 0.7 & 5.2 & 5.6 & 5.4 & 0.3 \\
\hline Second dorsal-fin posterior margin & 4.7 & 3 & $4.7-5.0$ & 4.8 & 0.2 & 5.9 & 5.6 & 5.8 & 0.2 \\
\hline Second dorsal-fin spine length & 5.0 & 3 & $4.3-5.3$ & 4.9 & 0.5 & 5.9 & 5.5 & 5.7 & 0.3 \\
\hline Second dorsal-fin spine base width & 0.7 & 3 & $0.7-0.8$ & 0.7 & 0.0 & 0.9 & 1.0 & 1.0 & 0.0 \\
\hline Pectoral-fin anterior margin length & 14.4 & 3 & $13.9-15.4$ & 14.4 & 0.7 & 16.1 & 13.2 & 14.7 & 2.1 \\
\hline Pectoral-fin inner margin length & 7.3 & 3 & $7.6-8.3$ & 7.8 & 0.4 & 8.1 & 9.3 & 8.7 & 0.8 \\
\hline Pectoral-fin base length & 4.7 & 3 & $4.3-9.4$ & 5.8 & 2.5 & 5.7 & 4.5 & 5.1 & 0.8 \\
\hline Pectoral-fin posterior margin length & 10.0 & 3 & $4.4-10.7$ & 8.4 & 2.8 & 12.3 & 10.1 & 11.2 & 1.5 \\
\hline Pelvic length & 10.7 & 3 & $9.5-9.9$ & 10.0 & 0.5 & 11.1 & 10.5 & 10.8 & 0.4 \\
\hline Pelvic-fin inner margin length & 5.4 & 3 & $4.5-5.1$ & 5.0 & 0.4 & 6.5 & 5.6 & 6.1 & 0.6 \\
\hline Dorsal caudal margin length & 20.6 & 3 & $20.0-21.5$ & 20.7 & 0.6 & 21.5 & 22.5 & 22.0 & 0.7 \\
\hline Preventral caudal margin length & 9.7 & 3 & $10.2-11.0$ & 10.3 & 0.5 & 11.5 & 10.8 & 11.2 & 0.5 \\
\hline Caudal fork width & 6.4 & 3 & $5.7-6.4$ & 6.1 & 0.3 & 6.8 & 6.2 & 6.5 & 0.4 \\
\hline Head width at nostrils & 7.1 & 3 & $6.9-7.2$ & 7.0 & 0.1 & 6.8 & 7.6 & 7.2 & 0.6 \\
\hline Head width at mouth & 11.4 & 3 & $11.2-12.1$ & 11.6 & 0.4 & 11.6 & 12.0 & 11.8 & 0.3 \\
\hline Head width & 13.1 & 3 & $11.8-14.1$ & 12.8 & 1.0 & 13.7 & 12.3 & 13.0 & 1.0 \\
\hline Trunk width & 11.7 & 3 & $9.8-12.3$ & 11.0 & 1.2 & 11.4 & 12.2 & 11.8 & 0.5 \\
\hline Abdomen width & 9.9 & 3 & $8.2-11.7$ & 9.7 & 1.5 & 8.5 & 10.1 & 9.3 & 1.1 \\
\hline Head height & 9.6 & 3 & $8.8-11.1$ & 10.0 & 1.0 & 10.9 & 10.5 & 10.7 & 0.3 \\
\hline Trunk height & 10.1 & 3 & $8.8-12.2$ & 10.6 & 1.5 & 12.1 & 12.5 & 12.3 & 0.3 \\
\hline Abdomen height & 10.9 & 3 & $8.6-13.4$ & 11.1 & 2.0 & 10.9 & 12.1 & 11.5 & 0.8 \\
\hline Clasper outer length & 3.3 & 2 & $1.0-3.4$ & 2.6 & 1.3 & 3.6 & - & 3.6 & - \\
\hline Clasper inner length & 6.3 & 2 & $1.5-5.9$ & 4.5 & 2.7 & 7.2 & - & 7.2 & - \\
\hline
\end{tabular}


found and elongate; apopyle broad, located anteriorly in the clasper groove; hypopyle broad, located anterior to rhipidion; rhipidion blade-like, thin and small, placed at the medial-distal end of the clasper.

Pelvic-caudal space 1.4 (1.1-1.6) times pectoral-pelvic space. Caudal keel evident laterally in the caudal peduncle from second dorsal fin free rear tip to caudal-fin origin. Caudal fin with conspicuously thin upper and lower caudal lobes (Fig. 1I, M); caudal fork concave with its width corresponding to $6.4 \%(5.7 \%-6.4 \%) \mathrm{TL}$; dorsal caudal margin straight and markedly elongate, its length 0.9 (0.9-1.0) times head length and $2.1(1.9-2.1)$ times length of preventral caudal margin; upper postventral margin straight on its upper half and slightly convex on its lower half; lower postventral margin convex; preventral caudal margin convex and short, its length 1.8 (2.12.3) times length of pelvic-fin inner margin; dorsal caudal tip rounded; ventral caudal tip markedly pointed.

Squamation (Fig. 7A, B). Dermal denticles lanceolate and imbricate, broad at the crown base (conspicuously broad in females); length of dermal denticles larger than its width (although it is about equal to its width in females); denticles with median cusp slightly pointed lateral cusps inconspicuous; median and lateral ridges thick; median and lateral ridge bifurcated anteriorly; anterior furrow conspicuously profound and oval.

Colouration (Fig. 1). Body brown dorsally, light brown laterally, although whitish latero-ventrally from the pelvic fin to the caudal fin and pale ventrally. Dorsal fins brownish, somewhat light brown at dorsal fin base; first dorsal-fin inner margin white; second dorsal-fin posterior margin fairly white at its lower half; second dorsal-fin apex somewhat dark brown. Dorsal-fin spines brownish anterior-laterally and white at the tip. Pectoral fins dark brown, whitish ventrally at pectoral-fin base; pectoral-fin posterior margin fairly white (not uniform); pectoral-fin apex broadly white. Pelvic fins brown and whitish at the pelvic-fin base; pelvic-fin anterior and posterior margins slightly white. Caudal fin dark brown at upper and lower caudal lobes, whitish over vertebral column; postventral caudal margins white, except at the caudal fork where it is dark brown; dorsal and ventral caudal tips broadly white; preventral caudal margin white; dark caudal stripe and bar inconspicuous; black upper caudal blotch somewhat evident in adults. Juveniles with body light brown dorsally, pale lateral and ventrally; first and second dorsal-fin posterior margins slightly white at its lower half; dorsal-fin apex blackish to dark brown at the tip; pectoral fins light brown to reddish-brown with pectoral-fin posterior margins and apex broadly white; pelvic fins light brown to reddish-brown; caudal fin dark brown with postventral caudal margins broadly white, except at the caudal fork; dorsal and ventral caudal tips broadly white; conspicuous upper black caudal blotch in the midline of dorsal caudal margin; lower caudal lobe with oblique black marking at the origin of lower caudal lobe; small black caudal bar at the caudal fork.
Vertebral counts (Table 6). 47 for holotype (44-48 for paratypes) monospondylous vertebrae; 93 (91-94) precaudal vertebrae; 122 (120-123) total vertebrae.

Etymology. This species is named after Dr. Shigeru M. Shirai, Japanese ichthyologist from Tokyo University of Agriculture, for his valuable contributions to Systematics of Squaliformes.

Vernacular. Shirai's spurdog; Hiretaka-tsunozame (Japanese).

Geographical distribution. This species is apparently a Japanese endemic, occurring in the shallow waters of the upper continental slope off Southern Japan in the North-western Pacific Ocean at 310-390 m depth (Fig. 8A).

Remarks. Squalus shiraii sp.nov. is often misidentified with $S$. mitsukurii due to similarities concerning the general shape of body, pectoral and caudal fins and snout length. However, it clearly differs from this species in addition to the characters provided in the diagnosis above by having an obtuse snout, dorsal and ventral caudal tips conspicuously pointed, upper and lower caudal lobes markedly tapered, pectoral fins conspicuously falcate, dermal denticles lanceolate and unicuspidate (vs. snout rounded, dorsal and ventral caudal tips rounded, upper and lower caudal lobes broad, pectoral fins not falcate, dermal denticles rhomboid and tricuspidate in S. mitsukurii). Squalus shiraii sp. nov. has body brown in colour, postventral and preventral caudal margins whitish, dorsal and ventral caudal tips broadly white and black upper caudal blotch evident in adults. S. mitsukurii has body conspicuously black to dark grey and caudal fins black throughout with post-ventral caudal margin fairly whitish and black upper caudal blotch not evident in adults.

Two recently described species, S. hawaiiensis and $S$. boretzi, are also morphologically similar to $S$. mitsukurii, but were supported as distinct according to molecular and morphological data in Daly-Engel et al. (2018) and Dolganov (2019). These species are apparently sympatric in the Hawaiian-Emperor Seamount Chain that runs across the North Pacific Ocean on about 6000 km from the Kamchatka Peninsula, Russia down to Hawaii, USA (Domier et al. 2017). Squalus shiraii sp. nov. share with $S$. hawaiiensis a more elongate snout that is more evident when considering the ratio prenarial length/inner-nostril labial furrow space $(1.2,1.1-1.3$ for $S$. shiraii sp. nov. vs. 1.3, 1.1-1.1 for $S$. hawaiiensis), body robust and presence of black upper caudal blotch, but morphometrics and vertebral counts support that they are conspicuously distinct as provided in the diagnosis above. Squalus shiraii sp. nov. may be further distinguished from the Hawaiian species by shorter pre-second dorsal length $60.5 \%, 61.1 \%-63.4 \%$ TL (vs. 63.6\%-67.0\% TL for S. hawaiiensis), pre-vent length $47.3 \%, 46.8 \%-48.7 \%$ TL (vs. $50.4 \%-53.6 \%$ TL for S. hawaiiensis), prepelvic length (44.1\%, 44.5\%-46.8\%TL (vs. $48.6 \%-52.4 \%$ TL for $S$. hawaiiensis) and interdorsal 
space $(23.8 \%, 24.0 \%-25.7 \%$ TL (vs. $26.7 \%-30.0 \%$ TL for $S$. hawaiiensis). Other differences include height of second dorsal fin, clasper length and head width at mouth (refer to Daly-Engel et al. 2018 for comparisons). Squalus shiraii $\mathrm{sp}$. nov. has lanceolate dermal denticles and dorsal fins markedly tall and upright (vs. tricuspidate, low and oblique dorsal fins in $S$. hawaiiensis). It also has shorter pectoral-caudal space than $S$. hawaiiensis and S. boretzi $(19.6 \%$, $17.1 \%-22.9 \%$ TL vs. $23.6 \%-27.7 \%$ TL for $S$. hawaiiensis vs. $25.2 \%-30.6 \%$ TL for $S$. boretzi). More detailed comparisons with $S$. boretzi were not accomplished here because we could not yet have access to the full original description of this species that is so far available in Russian only.

Some characteristics of $S$. shiraii are very similar to those of $S$. formosus. However, the Japanese species bears much smaller dorsal and pelvic fins and it has larger prefirst, pre-second and pre-caudal length. Other differences with the Taiwanese species include larger snout (preorbital and prenarial length), smaller and thinner second dorsal-fin spine, smaller spiracle and lower gill slits and dermal denticles lanceolate (vs. weakly tricuspidate; Fig. 7C, D). Squalus formosus is easily identified by the presence of white anterior margin of the first dorsal fin which is absent in the Japanese species. Vertebral counts further help to separate Squalus shiraii sp. nov. from other congeners, including precaudal vertebrae in $S$. mitsukurii, S. japonicus and S. brevirostris, as well as S. blainvillei, S. lalannei, S. montalbani, S. grahami, S. bahiensis, S. bassi, S. hawaiiensis and $S$. boretzi and total vertebrae in $S$. mitsukurii, S. japonicus, S. brevirostris, S. blainvillei, S. lalannei, $S$. montalbani, S. grahami, S. bahiensis, S. melanurus, $S$. hawaiiensis and $S$. boretzi. Other morphometric differences are also observed between $S$. shiraii and $S$. formosus and $S$. hawaiiensis regardless of maturity stage by more elongate snout (prenarial length 5.5\%, 5.4\%-5.6\% TL vs. $4.5 \%-4.9 \%$ TL for $S$. formosus vs. $4.8 \%-5.1 \%$ TL for $S$. hawaiiensis), first dorsal-fin height 8.1\%, 7.9\%-8.8\% TL (vs. 9.0\%-10.0\% TL for $S$. formosus vs. 6.9\%-7.7\% TL for $S$. hawaiiensis), second dorsal-fin anterior margin, its length $10.7 \%, 9.7 \%-11.3 \%$ TL (vs. $11.9 \%-12.6 \%$ TL for S. formosus vs. $6.7 \%-7.4 \%$ TL for $S$. hawaiiensis).

Comparative material. Squalus hawaiiensis: USNM 62450, adult female, unknown TL, Honolulu market, Oahu Island, Hawaii, USA; USNM 62467, adult female, $745 \mathrm{~mm}$ TL, locality same as USNM 62450. Type material of Squalus formosus: CSIRO H 6816-01 (holotype), adult male, $720 \mathrm{~mm}$ TL, Tashi fish Market, North-eastern Taiwan; CSIRO H 6292-10 (paratype), juvenile female, $335 \mathrm{~mm}$ TL, same locality as holotype.

\section{Squalus mitsukurii Jordan \& Snyder, 1903}

Figs 2, 3C, D, 7E, F, 8B; Tables 2, 6

Longnose spurdog; Futo-tsunozame (Japanese)

Squalus mitsukurii Jordan and Snyder 1901: 129 (name only); Jordan and Snyder 1903: 629; fig. 3 (original description, illustration; type by original designation; type locality: Misaki, Japan); Zhu 1960: 107, 109-110 (description; Northwest Pacific Ocean); Zhu et al. 1984: 284 (cited; Japan); Parin 1987: 48 (cited; Japan); Siming et al. 1988: 1 (listed; Northwest Pacific Ocean); Compagno and Niem 1998 (in part): 1230 (cited; Northwest Pacific Ocean); Zhu and Meng 2001: 311, 319-321 (cited, description; Northwest Pacific Ocean); Compagno 2002 (in part): 381, 385 (listed, description; Central Pacific Ocean); Nakabo 2002: 156 (listed; Tohoku District, Ryukyu Islands, Okinawa Trough, South China Sea); Compagno et al. 2005a (in part): 78 (description; Japan to Taiwan); Nakabo 2013: 196 (listed; Tohoku District, Ryukyu Islands, Okinawa Trough, South China Sea).

Squalus blainvillei: Zhu and Meng 2001: 311, 315-316 (cited, description; Japan).

Type material. AMNH 8822 (paratype), female, $248 \mathrm{~mm}$ TL; SU 7184 (paratype, identified as S. acanthias), male, $277 \mathrm{~mm}$ TL; SU 7748 (paratype), two females, 240-243 mm TL, male, $247 \mathrm{~mm}$ TL (All embryo paratypes taken from the holotype); SU 12793 (holotype), adult female, $710 \mathrm{~mm}$ TL, Honshu Island, Misaki, Japan, $35.159430^{\circ}, 139.493865^{\circ}, 14104 \mathrm{~m}$ depth (uncertain, according to CAS), collectors D. S. Jordan and J.O. Snyder in 1900; SU 12794 (paratype), adult male, $770 \mathrm{~mm}$ TL, data same as holotype.

Additional material. HUMZ 33680, adult female, $760 \mathrm{~mm}$ TL, East China Sea, $29^{\circ} 38^{\prime} \mathrm{N}, 134^{\circ} \mathrm{E}$; HUMZ 79798, adult female, $835 \mathrm{~mm}$ TL, Kyushu-Palau Ridge, $26^{\circ} 46.5^{\prime}-26^{\circ} 46.6^{\prime} \mathrm{N}, 135^{\circ} 20.3^{\prime}-135^{\circ} 20.8^{\prime} \mathrm{E}, 340-640 \mathrm{~m}$ depth; HUMZ 89858, juvenile female, $705 \mathrm{~mm}$ TL, off Hachijo-jima Island, Tokyo, Japan; HUMZ 97463, adult female, $800 \mathrm{~mm}$ TL, Ishikari Bay, Tokyo, Japan, $43^{\circ} 12.4^{\prime} \mathrm{N}, 141^{\circ} 08.5^{\prime} \mathrm{E}, 22 \mathrm{~m}$ depth; HUMZ 102986, adult female, $1005 \mathrm{~mm}$ TL, Central Pacific Ocean, near Northern Mariana Islands, $17^{\circ} 39.4^{\prime} \mathrm{N}, 145^{\circ} 50.3^{\prime} \mathrm{E}, 450 \mathrm{~m}$ depth; HUMZ 102987, adult female, $970 \mathrm{~mm}$ TL, Central Pacific Ocean, near Northern Mariana Islands, $19^{\circ} 09^{\prime} \mathrm{N}$, $142^{\circ} 59^{\prime} \mathrm{E}, 520 \mathrm{~m}$ depth; HUMZ 102988, adult female, 1025 mm TL, same locality as HUMZ 102987; HUMZ 113586, adult female, $1120 \mathrm{~mm}$ TL, off Shirahama, Shimoda, Shizuoka Prefecture, Japan, 45 m depth; HUMZ 113587, adult female, $990 \mathrm{~mm} \mathrm{TL}$, same locality as HUMZ 113586; HUMZ 113588 adult female, $960 \mathrm{~mm}$ TL, same locality as HUMZ 113586; NSMT-P 44097, adult female, $740 \mathrm{~mm}$ TL, Izu, Honshu, Japan; NSMT-P 44381, adult male, $770 \mathrm{~mm}$ TL, off Koura, Suruga Bay, Honshu, Japan; NSMT-P 65518, adult female, 1119 mm TL, Suno-saki Point, Boso Peninsula, Chiba Prefecture, Honshu, Japan, 25 m depth; NSMT-P 77187, adult male, 1000 mm TL, unknown locality; NSMT-P 97762, neonate male, 278 mm TL, Tsushima, Nagasaki Prefecture, Kyusyu, Japan; SU 14245, adult female, 580 mm TL, Hong Kong, China; ZUMT 1360, female embryo, 178 mm TL, off Atami, Kanagawa Prefecture, Japan; ZUMT 21114, female embryo, 190 mm TL, Nagasaki, Japan.

Diagnosis. Large-sized (710-1120 mm TL for adults) Squalus species that is distinguished from its regional 

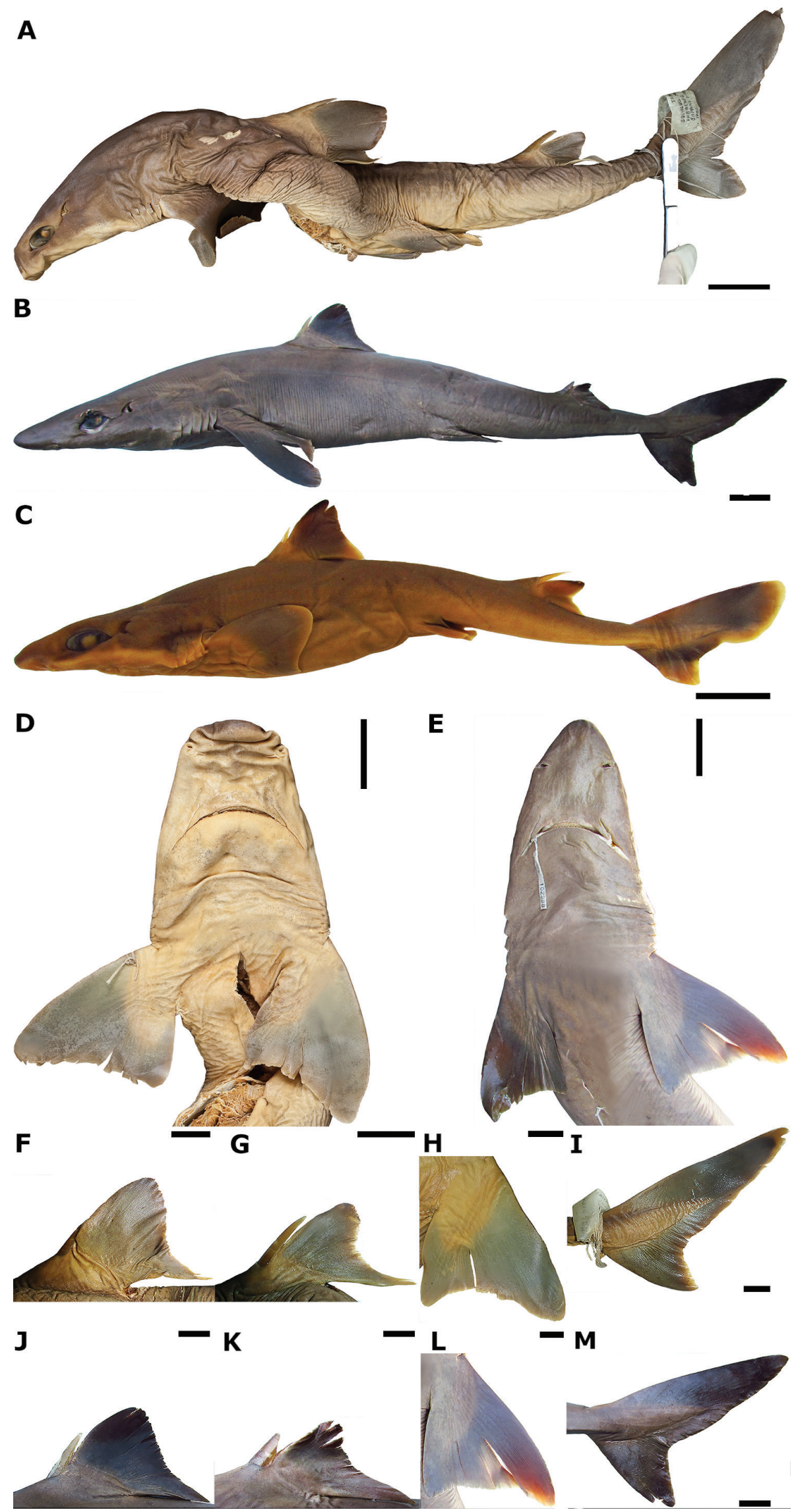

Figure 2. Squalus mitsukurii: lateral (A-C) and ventral (D, E) views; first (F, J) and second $(\mathbf{G}, \mathbf{K})$ dorsal fins; pectoral $(\mathbf{H}, \mathbf{L})$ and caudal (I, M) fins. SU 12794 (paratype), adult male, 770 mm (A, D, F-I); HUMZ 102988, adult female, 1025 mm TL (B, E, J-M); NSMT-P 97762, neonate male, $278 \mathrm{~mm}$ TL (C). Scale bars: $50 \mathrm{~mm}$ (A, B, D, E); $25 \mathrm{~mm}$ (C); $20 \mathrm{~mm}$ (F-M). 
congeners by: body dark grey to black in colour vs. body grey to light grey for $S$. japonicus vs. brownish-grey for $S$. brevirostris vs. brown for $S$. shiraii sp. nov.; dermal denticles tricuspidate and rhomboid (except for $S$. japonicus) vs. unicuspid and lanceolate. Squalus mitsukurii is separated from $S$. japonicus and $S$. brevirostris when adults by prenarial length $5.6 \%, 4.1 \%-5.6 \%$ TL (vs. 5.9\%, 5.9\%$6.8 \%$ TL for $S$. japonicus vs. $4.0 \%, 3.8 \%-4.3 \%$ TL for $S$. brevirostris) and from $S$. brevirostris and $S$. shiraii sp. nov. by smaller eyes, its length 3.6\%, 3.1\%-4.0\% TL (vs. $5.0 \%, 4.2 \%-5.1 \%$ TL for $S$. brevirostris vs. $4.9 \%, 4.4 \%-$ $4.9 \%$ TL for $S$. shiraii sp. nov.) and length of second dorsal-fin spine $0.8-1.1$ times length of first dorsal-fin spine (vs. 1.3-1.8 times for $S$. brevirostris vs. 1.2-1.3 times for $S$. shiraii sp. nov.). It is further distinct from $S$. japonicus by smaller preoral length and distance nostril-upper labial furrow $(10.3 \%, 7.6 \%-10.3 \%$ TL and $4.3 \%, 3.9 \%-4.5 \%$ TL vs. $10.7 \%, 10.7 \%-12.2 \%$ TL and $4.6 \%, 4.6 \%-5.1 \%$ TL for $S$. japonicus), more elongate first dorsal fin, its base length $8.2 \%, 7.6 \%-9.0 \%$ TL (vs. $6.7 \%, 6.6 \%-7.5 \%$ TL for $S$. japonicus), broader pectoral fins and head (pectoral-fin posterior margin length $11.7 \%, 9.9 \%-12.5 \% \mathrm{TL}$ and head width at mouth $12.2 \%, 10.9 \%-12.2 \%$ TL vs. $8.6 \%, 7.8 \%-9.2 \%$ TL and $9.7 \%, 9.7 \%-10.6 \%$ TL for $S$. japonicus). Squalus mitsukurii differs from $S$. brevirostris by: pectoral fin with free rear tips rounded and posterior margin straight to weakly concave vs. free rear tips pointed and posterior margin conspicuously concave for $S$. brevirostris; shorter pectoral-fin inner margin, its length 9.5\%, 7.7\%-9.5\% TL vs. $10.3 \%, 9.8 \%-11.2 \%$ TL for $S$. brevirostris; postventral caudal margins not uniformly white vs. uniformly white for $S$. brevirostris.

Description. External morphology. Body fusiform, markedly humped anteriorly from posterior margin of the spiracle to pelvic fin origin, turning slender posteriorly; body conspicuously robust and stout with head height 1.2 $(0.8-1.4)$ times trunk height and $1.6(0.9-1.7)$ times abdomen height and head width $1.2(1.0-1.6)$ times trunk width and 1.5 (1.1-2.6) times abdomen width (Fig. 2AC). Head flattened anteriorly, conspicuously elongate, its length $24.2 \%(20.4 \%-24.4 \%)$ TL and broad, its width at mouth $12.2 \%(10.4 \%-12.8 \%)$ TL. Snout rounded at the tip and noticeably elongate, its preorbital length $7.3 \%$ (6.9\%-10.7\%) TL; prenarial length 1.3 (1.1-1.4) times distance between nostrils to upper labial furrow and 0.5 (0.5-0.6) times preoral length; anterior margin of nostrils wide and bi-lobed; internarial space $1.3(0.8-1.4)$ times eye length (Fig. 2D, E). Eyes elliptical with anterior margin concave and posterior margin notched, placed laterally; eyes large, its length $4.1(1.6-3.0)$ times eye height. Prespiracular length $0.5(0.5-1.0)$ times prepectoral length and 1.8 (1.5-2.8) times preorbital length. Spiracles crescent, located laterally and behind the eyes; spiracles elongate, its length $0.4(0.2-0.4)$ times eye length. Prebranchial length $1.6(1.0-1.8)$ times prespiracular length.
Gill slits vertical and concave, placed anterior to origin of pectoral fins; gill slits very tall with first gill slit height $1.7 \%(1.5 \%-2.8 \%) \mathrm{TL}$; fifth gill slit $1.3(0.7-1.7)$ times taller than first gill slit.

Preoral length $1.2(1.0-1.5)$ times greater than mouth width. Mouth somewhat straight and conspicuously broad (mouth width 1.8, 1.5-2.1 times internarial width and 1.5, 1.2-1.8 times prenarial length); upper labial furrow elongate, its length $2.5 \%(2.1 \%-2.7 \%) \mathrm{TL}$, bearing a thin fold; lower labial furrow markedly elongate, lacking fold. Teeth unicuspid, flattened labial-lingually, similar in both jaws; teeth somewhat rectangular, very broad at the crown; cusp short, thick, pointed and oblique; mesial cutting edge convex and oblique; distal heel conspicuously rounded; mesial heel sharp; apron thick and small, placed more laterally in the upper teeth and in the midline of crown base in the lower teeth, slightly more elongate in the lower teeth than in the upper teeth; median teeth present on upper jaw in adult paratype only and distinct from the subsequent teeth by: teeth hexagonal with cusp and apron placed more medially; both mesial and distal heels pointed (Fig. 3C, D). One to two series of functional teeth on upper jaw and two series in lower jaw; 13-0-15 for holotype (13-1-13) teeth rows on upper jaw; 12-0-12 $(11-0-11)$ teeth rows on lower jaw.

Interdorsal space 2.0 (1.9-3.0) times larger than dorsal-caudal space and $0.9(0.9-1.3)$ times prepectoral length. Origin of first dorsal fin located prior to vertical traced at pectoral-fin free rear tips. First dorsal fin elongate, its length $1.1(1.0-1.3)$ times length of second dorsal fin; first dorsal-fin base length $0.8(0.7-1.3)$ times height of first dorsal fin. First dorsal fin conspicuously broad at fin web; first dorsal-fin anterior margin markedly convex and elongate, its length $12.0 \%(9.3 \%-13.2 \%)$ TL; first dorsal-fin posterior margin concave, its length 9.3\% (7.0\%-10.6\%) TL; first dorsal-fin inner margin elongate, its length $6.2 \%(5.0 \%-6.6 \%) \mathrm{TL}$; first dorsal-fin apex broadly rounded; first dorsal fin vertical and low, its height corresponding to $1.6(1.1-1.6)$ times length of first dorsal-fin inner margin and $1.3(0.8-1.4)$ times preorbital length (Fig. 2F, J). Origin of first dorsal-fin spine anterior to vertical traced at pectoral-fin free rear tips. First dorsal-fin spine thick, its width at base 1.0\% (0.5\%$1.0 \%$ ) TL; first dorsal-fin spine elongate, its length 3.9\% $(2.1 \%-5.4 \%) \mathrm{TL})$ and corresponding to $0.4(0.3-0.6)$ times first dorsal fin height, although not reaching first dorsal-fin apex. Pre-second dorsal length 2.5 (2.4-3.1) times prepectoral length. Origin of second dorsal fin behind a vertical traced at pelvic-fin free rear tips. Second dorsal fin broad at fin web with second dorsal-fin apex conspicuously rounded and lobe-like; second dorsal fin small, its length at base $0.9(0.7-1.3)$ times first dorsal fin base length; second dorsal fin oblique and low with second dorsal-fin height $1.3(0.8-1.4)$ times length of second dorsal-fin inner margin; second dorsal-fin anterior margin convex and elongate, its length $10.2 \%(7.7 \%-11.9 \%)$ TL; 
Table 2. External measurements of $S$. mitsukurii expressed as percentage of total length (\% TL). Values of the paratype of $S$. mitsukurii (SU 7184, identified as S. acanthias) and specimens of S. acutirostris are also provided. TL is expressed in millimeters. H: holotype; P: paratype; N: number of specimens; $x$ : mean; SD: standard deviation.

\begin{tabular}{|c|c|c|c|c|c|c|c|c|c|}
\hline & \multicolumn{5}{|c|}{ Squalus mitsukurii } & \multirow{2}{*}{$\begin{array}{c}\text { S. acanthias } \\
\text { SU } 7184\end{array}$} & \multicolumn{3}{|c|}{ S. acutirostris } \\
\hline & $\mathrm{H}$ & $\mathbf{N}$ & & $x$ & SD & & $\mathrm{H}$ & $\mathbf{P}$ & HUMZ 74990 \\
\hline Total length (mm) & 710.0 & 12 & $240.0-1120.0$ & 643.9 & 344.0 & 277.0 & 635.0 & 975.0 & 536.0 \\
\hline Precaudal length & 77.5 & 12 & $76.9-80.4$ & 78.3 & 1.1 & 79.4 & 81.9 & 79.0 & 78.0 \\
\hline Pre-second dorsal length & 61.0 & 12 & $58.4-63.9$ & 60.8 & 1.6 & 60.6 & 64.6 & 63.9 & 59.7 \\
\hline Pre-first dorsal length & 32.4 & 12 & $28.3-31.8$ & 30.7 & 1.1 & 36.1 & 32.3 & 31.8 & 28.4 \\
\hline Pre-vent length & 50.0 & 12 & $46.4-52.7$ & 49.2 & 1.9 & 50.5 & 52.8 & 46.7 & 47.6 \\
\hline Prepelvic length & 47.9 & 12 & $43.1-50.0$ & 46.8 & 1.8 & 47.7 & 49.3 & 47.2 & 45.0 \\
\hline Prepectoral length & 24.6 & 12 & $20.0-24.7$ & 22.4 & 1.4 & 21.5 & 24.3 & 23.6 & 21.6 \\
\hline Head length & 24.2 & 12 & $20.4-24.4$ & 22.7 & 1.2 & 22.2 & 24.4 & 22.6 & 22.1 \\
\hline Prebranchial length & 20.4 & 12 & $16.6-22.1$ & 19.9 & 1.5 & 18.9 & 20.9 & 17.9 & 19.1 \\
\hline Prespiracular length & 12.8 & 12 & $11.4-21.5$ & 13.6 & 2.8 & 12.4 & 13.9 & 10.6 & 12.8 \\
\hline Preorbital length & 7.3 & 12 & $6.9-10.7$ & 7.9 & 1.0 & 7.3 & 8.2 & 7.3 & 8.1 \\
\hline Prenarial length & 5.6 & 12 & $4.1-6.4$ & 5.1 & 0.6 & 5.3 & 4.7 & 5.1 & 4.7 \\
\hline Preoral length & 10.3 & 12 & $7.6-11.5$ & 9.8 & 1.0 & 10.5 & 10.4 & 9.5 & 10.2 \\
\hline Inner nostril-labial furrow space & 4.3 & 12 & $3.9-5.3$ & 4.5 & 0.4 & 5.1 & 6.9 & 8.6 & 5.9 \\
\hline Mouth width & 8.6 & 12 & $6.6-8.1$ & 7.7 & 0.5 & 7.1 & 9.4 & 8.2 & 7.2 \\
\hline Labial furrow length & 2.5 & 12 & $2.1-2.7$ & 2.4 & 0.2 & 2.3 & 2.2 & 1.9 & 2.3 \\
\hline Internarial space & 4.7 & 12 & $3.6-5.1$ & 4.3 & 0.4 & 4.0 & 3.6 & 3.7 & 4.7 \\
\hline Interorbital space & 9.3 & 12 & 7.7-9.8 & 8.9 & 0.7 & 8.2 & 9.6 & 9.2 & 8.5 \\
\hline Eye length & 3.6 & 12 & $3.1-5.2$ & 3.8 & 0.5 & 3.1 & 3.8 & 2.8 & 4.7 \\
\hline Eye height & 0.9 & 12 & $1.2-2.5$ & 1.7 & 0.4 & 2.0 & 1.6 & 1.7 & 2.1 \\
\hline Spiracle length & 1.3 & 12 & $0.8-1.9$ & 1.4 & 0.2 & 1.2 & 1.4 & 0.9 & 1.5 \\
\hline First gill-slit height & 1.7 & 12 & $1.5-2.8$ & 2.0 & 0.4 & 1.4 & 2.4 & 1.3 & 1.9 \\
\hline Fifth gill-slit height & 2.3 & 12 & $1.8-2.7$ & 2.4 & 0.2 & 1.9 & 3.3 & 1.6 & 2.2 \\
\hline Interdorsal space & 21.1 & 12 & $21.7-25.9$ & 23.6 & 1.6 & 18.0 & 28.3 & 24.6 & 23.5 \\
\hline Dorsal-caudal space & 10.6 & 12 & $8.1-11.9$ & 10.3 & 1.0 & 11.3 & 10.7 & 8.6 & 10.7 \\
\hline Pectoral-pelvic space & 21.8 & 12 & $18.2-25.3$ & 20.8 & 1.9 & 20.6 & 20.3 & 23.1 & 20.9 \\
\hline Pelvic-caudal space & 23.7 & 12 & $23.0-27.0$ & 24.6 & 1.1 & 22.7 & 26.8 & 21.3 & 24.1 \\
\hline First dorsal-fin length & 13.6 & 12 & $11.8-14.9$ & 13.8 & 0.9 & 13.9 & 15.0 & 14.1 & 14.2 \\
\hline First dorsal-fin anterior margin & 12.0 & 12 & $9.3-13.2$ & 11.6 & 1.1 & 12.0 & 10.1 & 10.9 & 10.8 \\
\hline First dorsal-fin base length & 8.2 & 12 & $5.3-9.0$ & 8.0 & 1.1 & 9.3 & 8.5 & 8.6 & 7.9 \\
\hline First dorsal-fin height & 9.8 & 12 & $6.9-9.8$ & 8.0 & 1.0 & 5.5 & 6.0 & 5.6 & 8.6 \\
\hline First dorsal-fin inner margin & 6.2 & 12 & $5.0-6.6$ & 6.0 & 0.5 & 5.1 & 5.7 & 5.6 & 6.3 \\
\hline First dorsal-fin posterior margin & 9.3 & 12 & $7.0-10.6$ & 8.9 & 0.9 & 5.2 & 8.7 & 7.8 & 9.8 \\
\hline First dorsal-fin spine length & 3.9 & 12 & $2.1-5.4$ & 3.5 & 1.1 & 2.1 & 2.8 & 2.7 & 3.2 \\
\hline First dorsal-fin spine base width & 1.0 & 12 & $0.5-1.0$ & 0.8 & 0.2 & 0.5 & 0.6 & 0.4 & 0.7 \\
\hline Second dorsal-fin length & 12.3 & 12 & $11.2-13.1$ & 12.1 & 0.6 & 13.3 & 10.6 & 9.8 & 12.2 \\
\hline Second dorsal-fin anterior margin & 10.2 & 12 & $7.7-11.9$ & 10.0 & 1.1 & 10.8 & 7.6 & 6.8 & 10.1 \\
\hline Second dorsal-fin base length & 7.2 & 12 & $6.2-8.2$ & 7.1 & 0.6 & 8.8 & 5.8 & 4.8 & 7.0 \\
\hline Second dorsal-fin height & 6.8 & 12 & $4.5-7.9$ & 5.7 & 1.0 & 3.9 & 3.3 & 3.6 & 6.0 \\
\hline Second dorsal-fin inner margin & 5.3 & 12 & $4.1-5.9$ & 5.0 & 0.6 & 4.8 & 5.0 & 5.0 & 4.9 \\
\hline Second dorsal-fin posterior margin & 6.3 & 12 & $4.5-6.3$ & 5.7 & 0.6 & 4.2 & 5.7 & 4.7 & 5.7 \\
\hline Second dorsal-fin spine length & 4.2 & 10 & $3.3-5.3$ & 4.1 & 0.6 & 3.7 & 2.8 & 1.9 & 4.8 \\
\hline Second dorsal-fin spine base width & 0.9 & 12 & $0.7-1.1$ & 0.8 & 0.1 & 1.0 & 0.6 & 0.5 & 0.7 \\
\hline Pectoral-fin anterior margin length & 15.2 & 12 & $12.3-17.7$ & 14.8 & 1.9 & 11.8 & 13.5 & 15.4 & 13.7 \\
\hline Pectoral-fin inner margin length & 9.5 & 12 & $7.7-9.5$ & 8.7 & 0.6 & 8.2 & 8.3 & 6.6 & 8.2 \\
\hline Pectoral-fin base length & 5.3 & 12 & $3.5-5.8$ & 4.7 & 0.6 & 4.1 & 5.4 & 6.1 & 4.1 \\
\hline Pectoral-fin posterior margin length & 11.7 & 12 & $9.2-12.5$ & 10.8 & 1.1 & 7.8 & 11.0 & 10.3 & 10.1 \\
\hline Pelvic length & 11.5 & 12 & $8.4-12.6$ & 10.5 & 1.1 & 9.9 & 10.2 & 8.7 & 10.2 \\
\hline Pelvic-fin inner margin length & 6.3 & 12 & $3.1-7.9$ & 5.2 & 1.1 & 4.8 & 5.0 & 5.1 & 4.9 \\
\hline Dorsal caudal margin length & 24.4 & 12 & $20.1-23.4$ & 21.8 & 1.2 & 20.9 & 16.7 & 20.5 & 22.1 \\
\hline Preventral caudal margin length & 12.1 & 12 & $10.7-12.4$ & 11.7 & 0.6 & 10.4 & 10.9 & 11.7 & 11.7 \\
\hline Caudal fork width & 7.0 & 12 & $6.1-7.7$ & 6.9 & 0.5 & 6.4 & 6.9 & 7.4 & 7.0 \\
\hline Head width at nostrils & 7.3 & 12 & $6.6-8.2$ & 7.3 & 0.5 & 7.1 & 6.8 & 5.2 & 7.6 \\
\hline Head width at mouth & 12.2 & 12 & $10.4-12.8$ & 11.4 & 0.7 & 9.1 & 11.3 & 10.7 & 11.8 \\
\hline Head width & 22.5 & 12 & $9.4-15.8$ & 13.3 & 3.2 & 10.5 & 11.0 & 13.3 & 12.5 \\
\hline Trunk width & 18.3 & 12 & $5.8-12.6$ & 10.8 & 2.9 & 7.4 & 7.9 & 9.4 & 10.6 \\
\hline Abdomen width & 15.5 & 12 & $5.4-11.0$ & 8.8 & 2.8 & 4.9 & 8.2 & 12.3 & 10.5 \\
\hline Head height & 12.7 & 12 & $8.3-12.5$ & 10.9 & 1.3 & 7.8 & 11.0 & 8.7 & 9.6 \\
\hline Trunk height & 10.3 & 12 & $5.7-14.1$ & 11.1 & 2.2 & 7.1 & 13.1 & 9.7 & 10.0 \\
\hline Abdomen height & 7.7 & 12 & $4.9-14.3$ & 10.0 & 3.2 & 5.8 & 13.2 & 8.2 & 9.6 \\
\hline Clasper outer length & - & 4 & $1.5-4.9$ & 3.4 & 1.6 & 1.6 & 4.3 & - & - \\
\hline Clasper inner length & - & 4 & $3.4-8.4$ & 5.6 & 2.4 & 3.1 & 7.4 & - & - \\
\hline
\end{tabular}




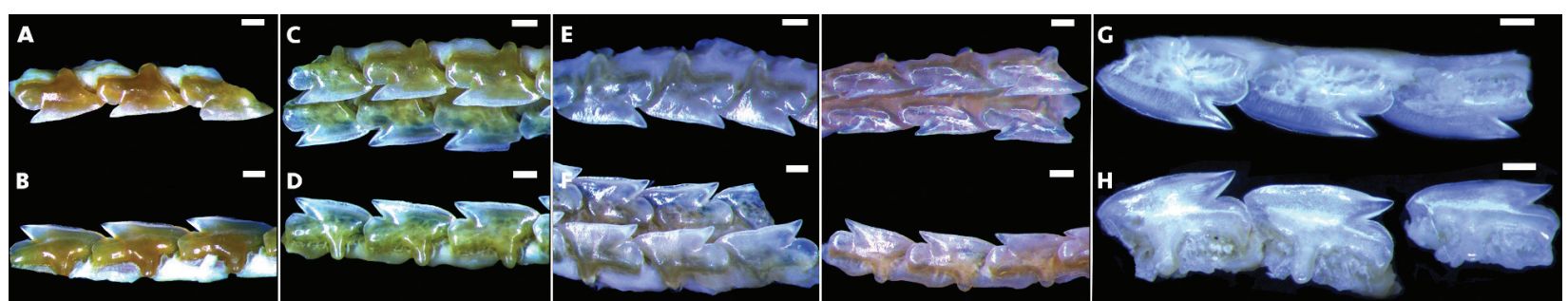

Figure 3. Upper (A, C, E, G, I) and lower (B, D, F, H, J) teeth of S. shiraii sp. nov. (A, B; HUMZ 80329), S. mitsukurii (C, D; HUMZ 33680), S. japonicus (E, F; HUMZ 189737), S. brevirostris (G, H; HUMZ 189762) and S. suckleyi (I, J; NSMT-P 92640). Scale bars: $1 \mathrm{~mm}$.

second dorsal-fin posterior margin concave and somewhat falcate, its length $6.3 \%(4.5 \%-6.3 \%) \mathrm{TL}$; second dorsal-fin inner margin length $5.3 \%(4.1 \%-5.9 \%)$ TL (Fig. 2G, K). Second dorsal-fin spine elongate, its length $0.6(0.5-1.0)$ times second dorsal fin height and $1.1(0.8-$ 1.9) times length of first dorsal-fin spine, although never reaching second dorsal-fin apex; second dorsal-fin spine thick, its base width $0.9 \%(0.7 \%-1.1 \%)$ TL.

Pectoral fins markedly broad and elongate (pectoral-fin posterior margin length $11.7 \%, 9.2 \%-12.5 \% \mathrm{TL}$ ), although never extending up to trunk height when adpressed laterally on body; pectoral-fin anterior margin straight; pectoral-fin inner margin convex; pectoral-fin posterior margin weakly concave medially; pectoral-fin apex rounded and lobe-like; pectoral-fin free rear tips rounded, reaching the same length when a horizontal line is traced at pectoral-fin apex (Fig. 2H, L); pectoral-fin anterior margin large, its length 1.6 (1.4-2.3) times greater than pectoral-fin inner margin length and $1.3(1.1-1.5)$ times pectoral-fin posterior margin length. Pectoral-pelvic space 0.9 (0.7-1.1) times pelvic-caudal space. Pelvic fins broad with pelvic-fin anterior and posterior margins convex; pelvic-fin free rear tips rounded and lobe-like; pelvic fin elongate, its length $11.5 \%(8.4 \%-12.6 \%)$ TL; pelvic fins nearer to second dorsal fin than to first dorsal fin. Adult males with clasper extremely robust and thick; clasper groove medial-dorsally and longitudinal, profound and short, not extending forward to inner margin of the clasper; clasper inner margin length $0.7-1.3$ times length of pelvic-fin inner margin; apopyle broad, placed anteriorly in the clasper groove; hypopyle wide, located just above the rhipidion; rhipidion blade-like and evidently slender and elongate, placed at the medial-distal end of the clasper.

Caudal keel prominent and situated laterally in the precaudal peduncle since insertion of second dorsal fin to behind origin of caudal fin. Caudal fin conspicuously slender in the upper caudal lobe; dorsal caudal margin somewhat convex proximally and conspicuously straight distally (Fig. 2I, M); caudal fin elongate with dorsal caudal margin length $1.0(0.9-1.1)$ times head length and 2.0 (1.7-2.0) times larger than length of preventral caudal margin; upper postventral caudal margin straight; lower postventral caudal margin somewhat convex; preventral caudal margin convex, its length 1.9 (1.5-3.8) times length of pelvic-fin inner margin; both posterior and ven- tral caudal tips rounded and thin; caudal fork discontinuous and conspicuously concave, its width $7.0 \%(6.1 \%-$ 7.7\%) TL.

Squamation (Fig. 7E, F). Dermal denticles tricuspid, rhomboid to heart-shaped and imbricate, conspicuously broad at the crown, its length equal to its width; cusps pointed and posterior; lateral cusps often inconspicuous and much shorter than median cusp; median cusp conspicuous and wide; median ridge prominent, tall and thick with anterior furrow oval and profound; anterior margin of the crown broad, convex and arrow-shaped with one small ridge on each side; one to two lateral ridges, thin and smaller than median ridge. Dermal denticles slightly larger and more imbricate in adults than juveniles.

Colouration (Fig. 2). Body black dorsally, dark grey ventrally and laterally since first dorsal fin insertion to origin of caudal fin. Dorsal fins blackish, dark grey at fin base near the dorsal-fin spines; lower half of anterior margin of dorsal fins whitish. Dorsal-fin spines brownish anteriorly and white posteriorly and at the tips. Pectoral fins black dorsally and ventrally, although dark grey near pectoral-fin inner margin; pectoral-fin posterior margin fairly white. Pelvic fins dark grey dorsally and ventrally, somewhat dark brown at the fin base; pelvic-fin posterior margins slightly white. Caudal fin black, dark grey near caudal fin origin and proximally over vertebral column; dorsal caudal margin dark grey proximally; postventral caudal margins slightly white, except near the caudal fork; preventral caudal margin blackish. Embryos, neonates and young juveniles differ from adults by having body light grey, dorsal fins light grey with dorsal-fin apex blackish and dorsal-fin free rear tips white, caudal fin light grey with postventral caudal margins broadly white, dark caudal bar conspicuous, black upper caudal blotch prominent and black caudal blotch at the base of lower caudal lobe, located horizontally.

Vertebral counts (Table 6). 68 (holotype) 70-72 (other material) diplospondylous vertebrae; 30 (26-31) caudal vertebrae; 116-117 total vertebrae.

Geographical distribution. Squalus mitsukurii is a regional endemic to the North-western Pacific Ocean with records along the coasts of China, Marianna Islands and Japan. It inhabits continental shelves and upper continental slopes within depth ranges of 22 to $640 \mathrm{~m}$ (Fig. 8B). 
This species possibly reaches depths of up to $980 \mathrm{~m}$ in the Western Pacific Ocean, but it is often caught between 180-300 m depth (Chen et al. 1979).

Remarks. Squalus mitsukurii has been misidentified with many nominal species in all oceans and, more particularly, with $S$. acutirostris, S. hawaiiensis, S. boretzi and $S$. shiraii sp. nov. in the North Pacific Ocean. Squalus mitsukurii exhibits smaller interdorsal space than S. hawaiiensis $(21.1 \%, 21.1 \%-25.9 \%$ TL vs. $26.7 \%-30.0 \% \mathrm{TL})$, larger first and second dorsal fins (first dorsal-fin base length 8.2\%, 7.6\%-9.0\% TL and second dorsal-fin base length 7.2\%, 6.8\%-7.9\% TL vs. $6.2 \%-7.4 \%$ TL and $4.9 \%-5.5 \%$ TL in S. hawaiiensis), which corroborates the findings of Daly-Engel et al. (2018). Torso measurements that are suggested as being diagnostic to separate these species in the referred study show minor overlapping and they must be used with caution despite range values of the direct measurements (e.g. pre-first and pre-second dorsal lengths, precaudal length) are somewhat higher in S. hawaiiensis. The Japanese species further exhibit more elongated pectoral fins, its inner margin 9.5\%, 7.7\%-9.5\% TL (vs. $6.4 \%-7.4 \%$ TL in $S$. hawaiiensis). Vertebral counts are congruent between these species, which is contrary to Daly-Engel et al. (2018) when comparing data taken from Chen et al. (1979). The latter work possibly included specimens of $S$. shiraii sp. nov. which is the only Japanese species with the highest number of vertebral counts. Morphologically similar Squalus species tend to exhibit overlapping of vertebral counts as seen here between S. mitsukurii, S. hawaiiensis and S. boretzi, with a few exceptions. Squalus mitsukurii may be separated from S. brevirostris, S. melanurus S. blainvillei and $S$. grahami by monospondylous vertebrae and from S. blainvillei, S. melanurus and S. montalbani by diplospondylous vertebrae and $S$. brevirostris, $S$. shiraii sp. nov., S. blainvillei and S. lalannei by total vertebrae. Precaudal vertebrae separates it from $S$. shiraii sp. nov., S. blainvillei, S. lalannei and S. montalbani.

\section{Squalus japonicus Ishikawa, 1908}

Figs 3E, F, 4, 7G, H, 8C; Tables 3, 6

Japanese spurdog; Togari-tsunozame (Japanese)

Squalus japonicus Ishikawa 1908: 71-73 (original description; not illustrated; type locality: Sagami Bay and Kagoshima, Japan; three syntypes cited and deposited in the Imperial Museum of Tokyo are considered lost: ZUMT uncatalogued, juvenile male, unknown TL, adult male, $700 \mathrm{~mm}$ TL, female, unknown TL); Chen et al. 1979: 26-42 (re-description; Japan); Parin 1987: 48-49 (cited; Japan); Compagno and Niem 1998: 1222, 1229 (listed, cited; Western North and Central Pacific Ocean); Zhu and Meng 2001: 311, 316-317 (cited, description; Northwest Pacific Ocean); Nakabo 2002: 155 (listed; Ryukyu Islands, Okinawa Trough, Southern Japan); Compagno et al. 2005a: 75-76 (description; Northwest Pacific Ocean); Ward et al. 2007: 118, 123, 129, 130 (cited; Taiwan); Naylor et al. 2012: 58 (cited; Taiwan); Nakabo 2013: 194 (listed; Ryukyu Islands, Okinawa Trough, Southern Japan); Straube et al. 2013: 264-265 (cited; Taiwan); Shinohara et al. 2014: 233 (cited; Japan).

Squalus suckleyi: Fowler 1941: 259 (listed; Japan).

Neotype. NSMT-P 44380, adult male, 645 mm TL, Senoumi Bank, Suruga Bay, Honshu, Japan, approximate coordinates $34^{\circ} 43^{\prime} \mathrm{N}$ and $138.33 \mathrm{E}, 270-300 \mathrm{~m}$ depth. Collected on 13 April 1982, collector Kazunari Yano.

Additional material. CSIRO H 6294-26, juvenile male, $440 \mathrm{~mm}$ TL, Tashi fish market, near I-Lan (NE coast), Taiwan; CSIRO H 6294-27, juvenile female, $360 \mathrm{~mm}$ TL, locality same as CSIRO H6294-26; CSIRO H 629431 , adult female, $540 \mathrm{~mm}$ TL, locality same as CSIRO H6294-26; HUMZ 40026, juvenile male, 215 mm TL, unknown locality; HUMZ 80223, adult male, unknown $\mathrm{TL}$, near Okinawa, Japan, $25^{\circ} 33.8^{\prime} \mathrm{N}, 126^{\circ} 25.2^{\prime} \mathrm{E}, 310 \mathrm{~m}$ depth; HUMZ 95213, unknown sex and TL, East China Sea, $27^{\circ} 46^{\prime} \mathrm{N}, 126^{\circ} 15.3^{\prime} \mathrm{E}, 296-465 \mathrm{~m}$ depth; HUMZ 189642, adult male, $530 \mathrm{~mm}$ TL, East China Sea; HUMZ 189673, juvenile female, $430 \mathrm{~mm}$ TL, East China Sea; HUMZ 189675, juvenile female, 277 mm TL, East China Sea; HUMZ 189676, juvenile male, $288 \mathrm{~mm}$ TL, East China Sea; HUMZ 189678, adult male, 545 mm TL, East China Sea; HUMZ 189682, juvenile male, 365 mm TL, East China Sea; HUMZ 189685, adult female, $512 \mathrm{~mm}$ TL, East China Sea; HUMZ 189687, juvenile male, 380 mm TL, East China Sea; HUMZ 189689, juvenile male, 276 mm TL, East China Sea; HUMZ 189693, juvenile female, $380 \mathrm{~mm}$ TL, East China Sea; HUMZ 189695, juvenile female, $290 \mathrm{~mm}$ TL, East China Sea; HUMZ 189696, juvenile male, $291 \mathrm{~mm}$ TL, East China Sea; HUMZ 189701, juvenile male, 287 mm TL, East China Sea; HUMZ 189705, juvenile male, $282 \mathrm{~mm}$ TL, East China Sea; HUMZ 189735, adult male, 518 mm TL, East China Sea; HUMZ 189737, adult male, 560 mm TL, East China Sea; HUMZ 189738, adult male, 535 mm TL, East China Sea; HUMZ 189739, adult male, 530 mm TL, East China Sea; HUMZ 191688, juvenile female, $340 \mathrm{~mm}$ TL, Okinawa, Japan; HUMZ 191689, juvenile male, 281 mm TL, Okinawa, Japan; HUMZ 191691, juvenile male, 338 mm TL, Okinawa, Japan; HUMZ 191693, juvenile female, 410 mm TL, Okinawa, Japan; HUMZ 191694, juvenile female, $440 \mathrm{~mm}$ TL, Okinawa, Japan; HUMZ 191695, juvenile male, 338 mm TL, Okinawa, Japan; HUMZ 191697, juvenile female, 330 mm TL, Okinawa, Japan; HUMZ 191698, juvenile male, 388 mm TL, Okinawa, Japan; HUMZ 191699, juvenile female, $410 \mathrm{~mm}$ TL, Okinawa, Japan; NMMB P 15491, adult male, 593 mm TL, Pingtung county, Taiwan; NMMB P 15691, adult male, $540 \mathrm{~mm}$ TL, locality same as NMMB P 15491; NMMB P 15696, adult male, 580 mm TL, locality same as NMMB P 15491; NMMB P 15698, juvenile female, 448 mm TL, locality same as NMMB P 15491; NMMB P 15699, adult male, $513 \mathrm{~mm}$ TL, locality same as NMMB P 15491; NMMB P 15700, juvenile female, 443 mm TL, locality same as NMMB P 15491; NSMT-P 47384, ju- 

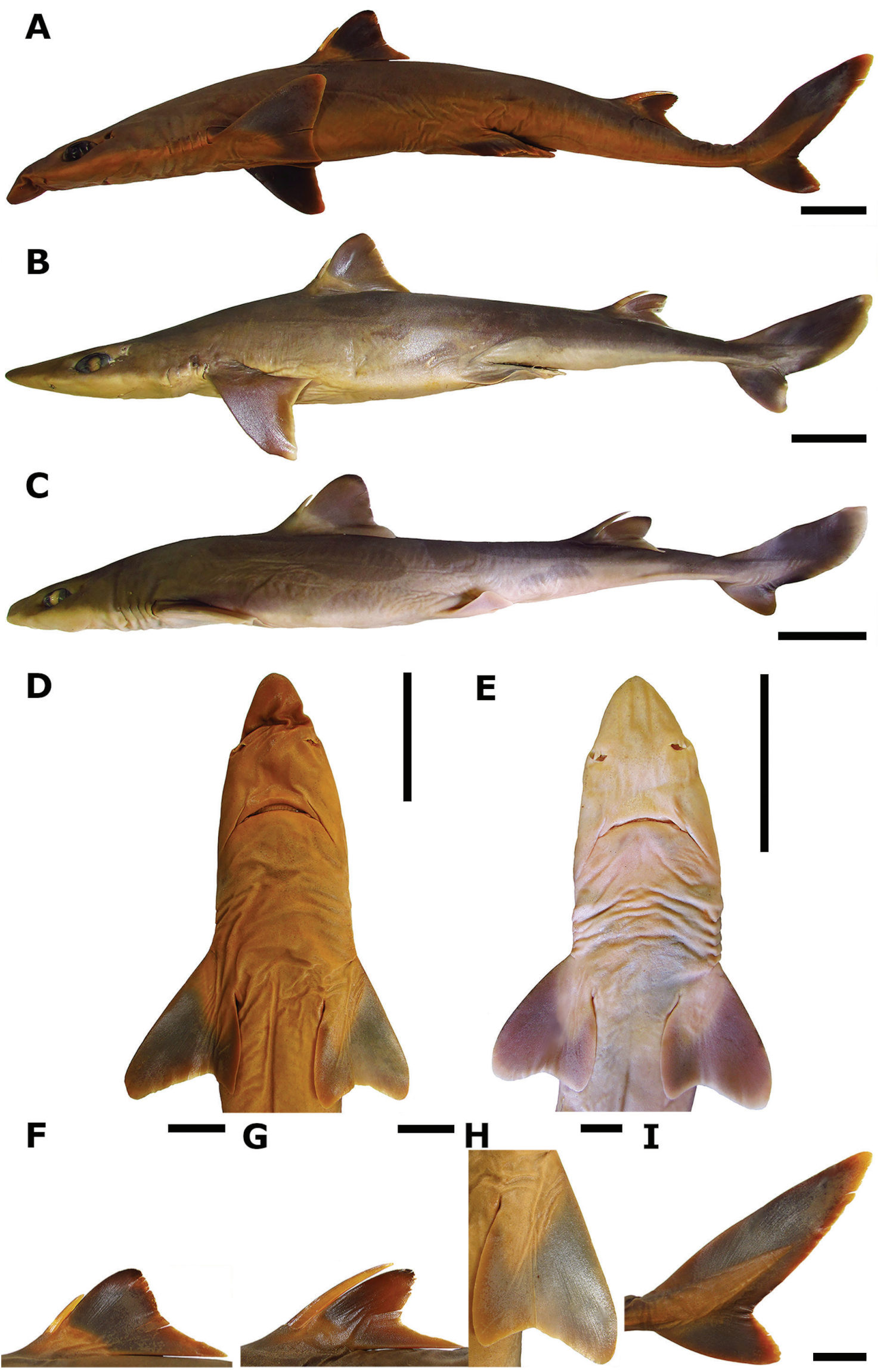

Figure 4. Squalus japonicus: lateral (A-C) and ventral (D, E) views; first (F) and second (G) dorsal fins; pectoral (H) and caudal (I) fins. NSMT-P 44380 (neotype), adult male, 645 mm TL (A, D, F-I); HUMZ 189738, adult male, 535 mm TL (B); HUMZ 189687, juvenile male, $380 \mathrm{~mm}$ TL (C, E). Scale bars: $50 \mathrm{~mm}(\mathbf{A}-\mathbf{E}) ; 20 \mathrm{~mm}(\mathbf{F}-\mathbf{I})$. 
venile female, $433 \mathrm{~mm}$ TL, Central Pacific Ocean; NSMT-P 67530, adult female, $560 \mathrm{~mm}$ TL, East China Sea, $31^{\circ} 21.31^{\prime} \mathrm{N}, 128^{\circ} 19.12^{\prime} \mathrm{E}, 254-260 \mathrm{~m}$ depth; NSMT-P 91127, juvenile female, $410 \mathrm{~mm}$ TL, Mimase Fishing Port, Kochi City, Kochi Prefecture, Shikoku, Japan; UF 44332, adult female, 490 mm TL, Seno-Umi, Honshu Island, Suruga Bay, Japan, 195 m depth.

Diagnosis. Single value corresponds to neotype and range values include all examined material from which data was taken. Species of Squalus clearly distinct from all its congeners (except with $S$. melanurus Fourmanoir, 1979 and $S$. nasutus) by a combination of characters: snout obtuse at tip and conspicuously elongate (prenarial length $5.9 \%-6.8 \%$ TL in adults); mouth markedly narrow, its width $1.6,1.0-1.6$ times prenarial length; pectoral fins conspicuously tapered (pectoral-fin posterior margin length $8.6 \%, 7.8 \%-9.2 \% \mathrm{TL}$ in adults); markedly elongate preoral length $10.7 \%, 10.7 \%-12.2 \%$ TL. Squalus japonicus is differentiated from its regional congeners $S$. mitsukurii, $S$. brevirostris, $S$. shiraii sp. nov. and $S$. acutirostris by head markedly tapered with head width at mouth $9.7 \%, 9.7 \%-10.6 \%$ TL (vs. $10.9 \%-$ $12.2 \%$ TL for $S$. mitsukurii vs. $10.7 \%-12.1 \%$ TL for $S$. brevirostris vs. $11.2 \%-11.6 \%$ TL for $S$. shiraii sp. nov. vs. $10.7 \%-11.6 \%$ TL for $S$. acutirostris) and caudal fin with conspicuous black caudal bar and black upper caudal blotch (vs. black caudal bar and black upper caudal blotch absent). It is separated from $S$. brevirostris and $S$. shiraii sp. nov. by more elongate pre-first dorsal length (31.8\%, 31.8\%-33.0\% TL vs. 29.6\%, 28.9\%-31.7\% TL for $S$. brevirostris and 30.5\%, 29.9\%-31.2\% TL for $S$. shiraii sp. nov.), larger preorbital length $(8.7 \%, 8.7 \%-$ $9.3 \%$ TL vs. $6.7 \%, 6.5 \%-7.5 \%$ TL for $S$. brevirostris vs. $7.9 \%, 7.4 \%-7.9 \%$ TL for $S$. shiraii sp. nov.) and from S. mitsukurii and S. shiraii sp. nov. by smaller first dorsal fin, its base length $6.7 \%, 6.6 \%-7.5 \%$ TL (vs. $8.2 \%$, $7.6 \%-9.0 \%$ TL for $S$. mitsukurii vs. $7.7 \%, 7.7 \%-8.7 \%$ TL for $S$. shiraii sp. nov.). It is also distinct from $S$. mitsukurii by larger inner nostril-labial furrow space $(4.6 \%$, $4.6 \%-5.1 \%$ TL vs. $4.3 \%, 3.9 \%-4.5 \%$ TL for $S$. mitsukurii) and from $S$. brevirostris by larger internarial space $(4.1 \%, 4.1 \%-4.7 \%$ TL vs. $3.6 \%, 3.4 \%-3.8 \%$ TL for $S$. brevirostris). It differs from $S$. shiraii sp. nov. by lower first dorsal fin, its height $6.6 \%, 6.6 \%-7.5 \%$ TL (vs. $8.1 \%, 7.9 \%-8.2 \%$ TL for $S$. shiraii sp. nov.) and lower number of precaudal and total vertebrae $(87,80-88$ and 116, 104-116 vs. 93, 91-94 and 122, 120-123 for S. shiraii sp. nov.). Squalus japonicus also differs from $S$. brevirostris by dermal denticles tricuspidate, pectoral-fin free rear tips rounded, postventral caudal margins not uniformly white (vs. unicuspid dermal denticles, pectoral-fin free rear tips pointed, postventral caudal margins uniformly white).

Description. Single values correspond to the neotype and ranges to all other examined material from which data were obtained.
External morphology. Medium sized species (512$645 \mathrm{~mm}$ maximum TL in adults), with body fusiform and skinny, somewhat arched from trunk to abdomen, turning slim to caudal fin; head height $0.9(0.8-1.1)$ times trunk and abdomen heights (Fig. 4A-C). Head compressed and very narrow, its width $1.2(1.1-1.3)$ times trunk width and 1.3 (1.0-1.7) times broader than abdomen width; head elongate, its length $23.6 \%(23.0 \%-24.5 \%$ TL). Snout conical and obtuse at the tip, conspicuously elongate (preorbital length $9.4 \%, 8.3 \%-10.1 \% \mathrm{TL}$ ); anterior margin of nostrils bi-lobed with first lobe larger than second lobe; nostrils much closer to the mouth than to the tip of the snout, its prenarial length $1.4(1.2-1.6)$ times the distance from nostril to upper labial furrow (Fig. 4D, E). Eyes oval with anterior margin convex and posterior margin notched; eyes elongate, its length 2.5 (2.0-3.5) times greater than its height. Prespiracular length $0.6(0.6-0.7)$ times prepectoral length. Spiracles crescent, placed laterally just posterior to the eyes; spiracles small, its length $0.3(0.2-0.4)$ times eye length. Prebranchial length 1.4 (1.4-1.5) times larger than prespiracular length. Gill slits convex, located anterior to origin of pectoral fins; gill slits vertical and low with height of fifth gill slit $1.2(0.9-1.6)$ times height of first gill slit.

Preoral length 1.8 (1.4-2.7) times greater than mouth width. Mouth markedly arched and narrow, its width 1.5 (1.0-1.6) times broader than internarial width and $1.0(0.6-1.3)$ times prenarial length; upper labial furrow elongate, its length $2.1 \%(1.9 \%-2.4 \% \mathrm{TL})$, with fold very thin; lower labial furrow also elongate, although without fold. Teeth unicuspid and tiny on both jaws, although upper teeth smaller than lower teeth; cusp short and oblique, thicker on lower teeth than upper teeth; mesial cutting edge convex; mesial heel markedly notched; distal heel rounded; apron thick and short, somewhat conical in lower teeth (Figs 3E-F). Two series of functional teeth on each jaw; 13-0-13 teeth rows for neotype (13-0-13 for other material) in upper jaw; 10-0-10 (11-0-11) teeth rows in lower jaw.

Pre-first dorsal length 1.4 (1.3-1.5) times larger than prepectoral length. Origin of first dorsal fin prior to the vertical line traced at pectoral-fin free rear tips. First dorsal fin evidently slender at fin web (broader in young juveniles); first dorsal-fin anterior margin convex; first dorsal-fin posterior margin almost straight, although falcate distally; first dorsal-fin apex markedly rounded; first dorsal-fin free rear tip pointed (Fig. 4F); first dorsal fin short, its base length $1.0(0.8-1.1)$ times height of first dorsal-fin, its length $1.0(0.8-1.1)$ times length of second dorsal fin; first dorsal fin low, its height 1.3 (1.1-1.6) times length of first dorsal-fin inner margin; first dorsal-fin inner margin small, its length 5.6\% (5.1\%$6.6 \%)$ TL. First dorsal-fin spine narrow, its base width $0.6 \%(0.5 \%-0.8 \%)$ TL and very short (its length $2.9 \%$, $2.0 \%-3.5 \% \mathrm{TL})$, comprising $0.4(0.3-0.5)$ times first dorsal-fin height. Interdorsal space $1.0(0.8-1.1)$ times prepectoral length and $2.2(1.8-2.4)$ times larger than dorsal caudal space. Pre-second dorsal length 2.7 (2.6-2.8) 
Table 3. External measurements for S. japonicus expressed as percentage of total length (\% TL). Morphometric data of $S$. nasutus is also provided for comparisons. TL is expressed in millimeters. N: number of specimens; $x$ : mean; SD: standard deviation.

\begin{tabular}{|c|c|c|c|c|c|c|c|}
\hline & \multicolumn{3}{|c|}{ S. japonicus } & \multicolumn{2}{|r|}{ S. nasutus } & \multirow[t]{2}{*}{$x$} & \multirow[t]{2}{*}{ SD } \\
\hline & Neotype & $\mathbf{N}$ & & $\mathbf{N}$ & & & \\
\hline Total length (mm) & 645.0 & 15 & $281.0-560.0$ & 9 & $362.0-580.0$ & 444.9 & 97.2 \\
\hline Precaudal length & 79.7 & 15 & $77.3-80.7$ & 9 & $79.6-81.5$ & 79.9 & 0.9 \\
\hline Pre-second dorsal length & 62.0 & 15 & $59.1-62.4$ & 9 & $60.8-64.6$ & 61.6 & 1.1 \\
\hline Pre-first dorsal length & 31.8 & 15 & $30.0-33.8$ & 9 & $31.2-32.9$ & 32.2 & 0.7 \\
\hline Pre-vent length & 47.3 & 15 & $47.0-50.4$ & 9 & $46.1-51.7$ & 48.4 & 1.2 \\
\hline Prepelvic length & 43.7 & 15 & $44.8-47.9$ & 9 & $44.5-48.7$ & 45.9 & 1.1 \\
\hline Prepectoral length & 22.5 & 15 & $22.0-24.0$ & 9 & $22.8-25.2$ & 23.3 & 0.7 \\
\hline Head length & 23.3 & 15 & $23.0-24.5$ & 9 & $23.5-26.8$ & 23.9 & 0.8 \\
\hline Prebranchial length & 18.7 & 15 & $19.4-21.6$ & 9 & $20.3-22.1$ & 20.5 & 0.8 \\
\hline Prespiracular length & 12.8 & 15 & $13.5-15.4$ & 9 & $13.7-15.6$ & 14.5 & 0.6 \\
\hline Preorbital length & 8.7 & 15 & $8.3-10.1$ & 9 & $9.2-10.4$ & 9.5 & 0.5 \\
\hline Prenarial length & 5.9 & 15 & $5.8-7.2$ & 9 & $6.6-7.5$ & 6.8 & 0.4 \\
\hline Preoral length & 10.7 & 15 & $11.1-12.5$ & 9 & $11.8-13.0$ & 12.0 & 0.5 \\
\hline Inner nostril-labial furrow space & 4.6 & 15 & $4.4-5.5$ & 9 & $4.5-5.6$ & 5.0 & 0.3 \\
\hline Mouth width & 6.4 & 15 & $4.3-7.8$ & 9 & $6.4-7.3$ & 6.7 & 0.7 \\
\hline Labial furrow length & 2.0 & 15 & $1.9-2.4$ & 9 & $1.9-2.4$ & 2.1 & 0.2 \\
\hline Internarial space & 4.1 & 15 & $4.1-5.4$ & 9 & $4.3-5.4$ & 4.6 & 0.4 \\
\hline Interorbital space & 7.5 & 15 & $7.6-9.1$ & 9 & $8.2-9.2$ & 8.4 & 0.5 \\
\hline Eye length & 3.8 & 15 & $3.8-5.2$ & 9 & $4.2-5.3$ & 4.5 & 0.4 \\
\hline Eye height & 1.9 & 15 & $1.4-2.2$ & 9 & $1.8-2.2$ & 1.9 & 0.2 \\
\hline Spiracle length & 1.2 & 15 & $1.0-1.8$ & 9 & $1.1-1.5$ & 1.4 & 0.2 \\
\hline First gill-slit height & 1.3 & 15 & $1.3-2.0$ & 9 & $1.3-1.8$ & 1.5 & 0.2 \\
\hline Fifth gill-slit height & 1.6 & 15 & $1.5-2.1$ & 9 & $1.8-2.2$ & 1.9 & 0.2 \\
\hline Interdorsal space & 24.8 & 15 & $18.4-23.5$ & 9 & $23.0-25.2$ & 23.1 & 1.5 \\
\hline Dorsal-caudal space & 11.4 & 15 & $9.5-11.1$ & 9 & $10.9-13.2$ & 10.8 & 0.8 \\
\hline Pectoral-pelvic space & 18.6 & 15 & $18.7-22.6$ & 9 & $17.8-23.1$ & 19.8 & 1.4 \\
\hline Pelvic-caudal space & 27.9 & 15 & $23.6-28.0$ & 9 & $25.0-28.8$ & 26.4 & 1.3 \\
\hline First dorsal-fin length & 12.6 & 15 & $12.0-14.7$ & 9 & $11.8-13.3$ & 12.7 & 0.7 \\
\hline First dorsal-fin anterior margin & 10.1 & 15 & $9.6-13.3$ & 9 & $9.9-11.5$ & 10.7 & 0.8 \\
\hline First dorsal-fin base length & 6.7 & 15 & $6.2-8.6$ & 9 & $6.8-8.0$ & 7.2 & 0.5 \\
\hline First dorsal-fin height & 6.6 & 15 & $6.6-10.0$ & 9 & $6.9-7.7$ & 7.4 & 0.7 \\
\hline First dorsal-fin inner margin & 5.9 & 15 & $5.1-6.6$ & 9 & $5.0-5.7$ & 5.5 & 0.4 \\
\hline First dorsal-fin posterior margin & 7.8 & 15 & $5.7-10.0$ & 9 & $6.8-8.8$ & 7.6 & 0.9 \\
\hline First dorsal-fin spine length & 3.8 & 14 & $2.0-3.5$ & 8 & $2.4-3.8$ & 3.0 & 0.5 \\
\hline First dorsal-fin spine base width & 0.7 & 15 & $0.5-0.8$ & 9 & $0.6-0.8$ & 0.7 & 0.1 \\
\hline Second dorsal-fin length & 12.8 & 15 & $12.3-16.1$ & 9 & $10.1-13.0$ & 12.9 & 1.2 \\
\hline Second dorsal-fin anterior margin & 11.1 & 15 & $10.5-14.5$ & 9 & $8.1-11.2$ & 11.0 & 1.3 \\
\hline Second dorsal-fin base length & 7.7 & 15 & $6.9-10.6$ & 9 & $5.9-8.4$ & 8.0 & 1.1 \\
\hline Second dorsal-fin height & 6.1 & 15 & $5.4-7.3$ & 9 & $4.8-5.4$ & 5.9 & 0.7 \\
\hline Second dorsal-fin inner margin & 4.9 & 15 & $4.3-5.5$ & 9 & $4.0-4.9$ & 4.9 & 0.4 \\
\hline Second dorsal-fin posterior margin & 4.4 & 15 & $4.4-6.1$ & 9 & $4.5-5.5$ & 5.1 & 0.5 \\
\hline Second dorsal-fin spine length & 5.6 & 14 & $4.1-5.6$ & 9 & $3.9-4.9$ & 4.7 & 0.6 \\
\hline Second dorsal-fin spine base width & 0.7 & 15 & $0.7-1.0$ & 9 & $0.7-1.1$ & 0.8 & 0.1 \\
\hline Pectoral-fin anterior margin length & 13.1 & 15 & $10.9-14.2$ & 9 & $12.4-14.2$ & 12.7 & 0.9 \\
\hline Pectoral-fin inner margin length & 7.5 & 15 & $7.8-8.9$ & 9 & $7.2-8.5$ & 8.2 & 0.5 \\
\hline Pectoral-fin base length & 4.6 & 15 & $3.8-4.7$ & 9 & $4.1-5.0$ & 4.4 & 0.4 \\
\hline Pectoral-fin posterior margin length & 8.6 & 15 & $6.8-10.1$ & 9 & $6.2-8.9$ & 7.9 & 0.9 \\
\hline Pelvic length & 11.7 & 15 & $9.6-12.4$ & 9 & $9.3-12.6$ & 10.9 & 0.8 \\
\hline Pelvic-fin inner margin length & 7.3 & 15 & $4.6-6.1$ & 9 & $4.3-7.0$ & 5.5 & 0.8 \\
\hline Dorsal caudal margin length & 20.2 & 15 & $19.6-21.8$ & 9 & $18.1-19.8$ & 19.9 & 0.8 \\
\hline Preventral caudal margin length & 10.9 & 15 & $6.6-11.7$ & 9 & $10.2-12.4$ & 10.9 & 1.0 \\
\hline Caudal fork width & 6.2 & 15 & $6.0-7.4$ & 9 & $6.2-7.5$ & 6.7 & 0.4 \\
\hline Head width at nostrils & 6.3 & 15 & $6.8-8.1$ & 9 & $6.9-8.3$ & 7.4 & 0.5 \\
\hline Head width at mouth & 9.7 & 15 & $10.0-11.9$ & 9 & $10.2-11.3$ & 10.7 & 0.5 \\
\hline Head width & 11.6 & 15 & $11.0-13.0$ & 9 & $11.2-12.7$ & 11.7 & 0.5 \\
\hline Trunk width & 9.7 & 15 & $9.1-10.8$ & 9 & $8.7-12.0$ & 10.0 & 0.7 \\
\hline Abdomen width & 8.6 & 15 & $6.9-10.9$ & 9 & $6.3-9.2$ & 8.8 & 1.2 \\
\hline Head height & 9.4 & 15 & $8.5-10.6$ & 9 & 8.9-9.8 & 9.5 & 0.6 \\
\hline Trunk height & 10.2 & 15 & $8.3-11.6$ & 9 & $8.9-10.3$ & 10.0 & 0.9 \\
\hline Abdomen height & 10.0 & 15 & $8.7-12.2$ & 9 & $8.3-12.2$ & 10.1 & 1.1 \\
\hline Clasper outer length & 4.8 & 6 & $1.4-4.5$ & 4 & $2.1-5.6$ & 3.3 & 1.7 \\
\hline Clasper inner length & 7.5 & 6 & $2.8-7.9$ & 4 & $4.3-9.1$ & 5.8 & 2.6 \\
\hline
\end{tabular}


times prepectoral length. Second dorsal fin slender at fin web; second dorsal-fin anterior margin concave; second dorsal-fin posterior margin concave and deeply falcate; second dorsal-fin apex rounded and lobe-like; second dorsal-fin free rear tip pointed (Fig. 4G); second dorsal fin short, its length $2.1(1.8-2.5)$ times second dorsal-fin height; second dorsal-fin inner margin small, its length $5.0 \%(4.3 \%-5.5 \%)$ TL; second dorsal fin low, its height $1.3(1.2-1.4)$ times length of second dorsal-fin inner margin. Second dorsal-fin spine slender, its base width $0.8 \%$ $(0.7 \%-1.0 \%) \mathrm{TL}$; second dorsal-fin spine small, its length $4.9 \%(4.1 \%-5.6 \%) \mathrm{TL}$, corresponding to $0.8(0.6-1.0)$ times height of second dorsal fin, almost reaching its apex; second dorsal-fin spine 1.7 (1.3-2.2) times larger than first dorsal-fin spine.

Pectoral fins conspicuously narrow (pectoral-fin posterior margin length $7.9 \%, 6.8 \%-10.1 \% \mathrm{TL}$ ); pectoral-fin anterior and inner margins convex; pectoral-fin posterior margin concave (deeply concave in juveniles); pectoral-fin anterior margin length $1.6(1.4-1.7)$ times length of pectoral-fin posterior margin and 1.5 (1.3-1.7) times length of pectoral-fin inner margin; pectoral-fin apex and free rear tips rounded; pectoral-fin free rear tips lobe-like and reaching the horizontal line traced at pectoral-fin apex (in juveniles, pectoral-fin free rear tips transcend the pectoral-fin apex) (Fig. 4H). Pectoral-pelvic space 0.8 (0.7-0.8) times pelvic-caudal space. Pelvic-caudal space 1.2 (1.1-1.4) times larger than interdorsal space; pelvic fins in the midline between two dorsal fins, slightly nearer to first dorsal fin. Pelvic fins narrow with margins straight; pelvic-fin free rear tips pointed; pelvic fins short, its length $10.8 \%(9.6 \%-12.4 \%)$ TL. Clasper thin and small, slightly transcending pelvic-fin free rear tips; clasper inner margin length $0.8(0.5-1.3)$ times length of pelvic inner margin; clasper groove dorsal and longitudinal, elongate and open; apopyle rounded, located anteriorly in the clasper groove; hypopyle constricted, located posteriorly in the clasper groove; rhipidion very thin and soft, flap-like, placed medially at distal end of the clasper.

Caudal keel prominent and lateral since insertion of second dorsal fin to behind origin of caudal fin. Caudal fin small with dorsal caudal margin length $0.9(0.8-0.9)$ times head length and 1.9 (1.8-3.1) times length of preventral caudal margin; dorsal and ventral caudal lobes slender (dorsal lobe rectangular in juveniles); dorsal caudal tip pointed; dorsal caudal margin slightly convex; upper postventral caudal margin straight; lower postventral caudal margin convex (Fig. 4I); preventral caudal margin convex and short, its length 2.0 (1.4-2.4) times greater than length of pelvic-fin inner margin; ventral caudal tip rounded; caudal fork discontinuous, markedly concave and narrow, its width $6.6 \%(6.0 \%-7.4 \%)$ TL.

Squamation (Fig. 7G, H). Dermal denticles tricuspid and markedly imbricated, its length slightly greater than its width; denticles conspicuously broad at the crown base; cusps posterior, prominent and pointed; lateral cusps shorter than median cusp, forming well-marked concavity with median cusp; median ridge thick and elon- gate; one to two lateral ridges, very thin, almost equal in length to median ridge; anterior furrow shallow with one small ridge on each side; anterior margin of the crown arrow-shaped. In juveniles, denticles are slender at the crown base, with cusps conspicuously pointed and more elongate and anterior margin of the crown tapered.

Colouration (Fig. 4). Body grey to light grey dorsally and white laterally from insertion of pectoral fins to origin of caudal fin. Dorsal fins greyish, darker at its apex to the upper half of the posterior margin, whitish at fin base and free rear tips; dorsal-fin spine brown anteriorly, white posteriorly and at the tips. Pectoral fins grey, slightly darker near pectoral-fin anterior margin; pectoral-fin posterior margin strongly white and uniform. Pelvic fins grey dorsally and completely pale ventrally; pelvic-fin posterior margin white. Caudal fin mostly greyish, light grey over vertebral column; dorsal caudal margin whitish proximally; upper postventral caudal margin white, lower postventral caudal margin broadly white; lower caudal lobe mostly white; preventral caudal margin whitish; black caudal stripe conspicuous; black upper caudal blotch conspicuous in the upper caudal lobe, nearest to dorsal caudal tip; black caudal bar oblique, placed in the caudal fork to midline of upper postventral caudal margin; dorsal and ventral caudal tips broadly white. Embryos, neonates and young juveniles exhibit body light grey with caudal bar and upper caudal blotch more conspicuous than in adults.

Vertebral counts (Table 6). 87 (neotype) 80-88 (other material) precaudal vertebrae; 73 (65-75) diplospondylous vertebrae; 115 (104-116) total vertebrae.

Geographical distribution. Squalus japonicus is a regional endemic species occurring in the North-western Pacific Ocean from Southern Japan, China to Taiwan (Fig. $8 \mathrm{C})$. It is found on the upper continental slope of tropical and subtropical areas, ranging between 195-520 m depth, although it also has been reported at $52 \mathrm{~m}$ depth only in Weigmann (2016). This species also occurs in Korean waters (Chen et al. 1979).

Remarks. Ishikawa (1908) mentioned three syntypes of $S$. japonicus that were deposited at the Zoological University Museum of Tokyo (formerly Imperial Museum of Tokyo). A visit to the fish collection from ZUMT was undertaken in 2013 to verify the existence of the type material. However, it was noticed that there was a single specimen of $S$. japonicus held in the museum, assuming that syntypes are lost and no longer exist. Dr. M. Nakae (NSMT, person. comm. 2014) stated that the NSMT fish collection has never been in possession of this material and ratified that the syntypes are lost. Squalus japonicus has been misidentified in waters outside Japan (e.g. Vietnam by Quang et al. 2013) and many morphological variations were reported earlier in Compagno et al. (2005b) and Last et al. (2007b) for material from Indonesia, Philippines and Western Australia, compromising the correct application of this nominal species. To clarify 
the taxonomic status of $S$. japonicus across the Western Pacific Ocean, the designation of a neotype along with a re-description of this species is provided herein. The neotype was collected from Southern Japan (Senoumi Bank, Suruga Bay), which is within the known range distribution of $S$. japonicus, based on the examined material, the original description of $S$. japonicus and information in literature. The designation of the neotype was further based on the sex, maturity and total length that were approximate to those provided for a syntype of $S$. japonicus in Ishikawa (1908). The neotype is securely deposited in the scientific fish collection from the NSMT in Tokyo at the Department of Zoology for preservation and accessibility purposes. This species is easily separated from its congeners through many characteristics of the external morphology, morphometrics and meristic data, including shape of head, snout and pectoral fins, length of snout, width of mouth and preoral length and several other external measurements as shown in the diagnosis above. The current diagnosis and re-description of $S$. japonicus are congruent with observations of Ishikawa (1908) and Chen et al. (1979) with the exception of the presence of two hooks in the clasper. These cartilages of the clasper probably concern the accessory terminal 3 cartilage (spur) and dorsal terminal cartilage (claw) that are commonly noticed in species of Squalus. Differences in morphometrics are observed between the data provided in Ishikawa (1908) and those from the present analysis, but this is due to divergences of the applied methodology.

This species together with $S$. melanurus and the Australian species $S$. nasutus, comprise the $S$. japonicus subgroup of species whose members are characterised by having a conspicuously elongate and obtuse snout, thin body, mouth very narrow and pectoral fins tapered (Viana and Carvalho 2018a). The Japanese species is more closely related to $S$. nasutus according to morphological and genetic data (Last et al. 2007b). Shape of dermal denticles, dentition and shape of dorsal, pectoral, pelvic and caudal fins are intrinsically similar between these two species. Morphometrics are also mostly congruent, although differences in measurements are apparent when adults are compared: second dorsal fin height 5.4\%-6.5\% TL for S. japonicus vs. 4.9\%-5.3\% TL for S. nasutus; length of second dorsal-fin anterior margin $10.8 \%-12.7 \%$ TL vs. $8.6 \%-10.5 \%$ TL; length of second dorsal-fin spine $4.7 \%-5.6 \%$ TL vs. $3.9 \%-4.4 \%$ TL; length of dorsal caudal margin $19.6 \%-20.4 \%$ TL vs. $18.1 \%-19.4 \%$ TL. Preoral length, eye length, pectoral-fin inner margin length, trunk height and width overlaps between these two species which contradicts Last et al. (2007b) in supporting these measurements as diagnostic characters for these species. Vertebral counts differ between $S$. japonicus and $S$. nasutus as well: monospondylous (41-43), precaudal (84-88) vertebrae (vs. 37-40; 80-83 for those from Australia). These results are congruent with recent molecular analyses of the cytochrome c oxydase 1 (COI) and NADH dehydrogenase 2 (NAD2) mitochondrial genes in Ward et al. (2007), Naylor et al. (2012), Straube et al. (2013), Veríssimo et al. (2017) and Daly-Engel et al. (2018) which support genetic divergence between $S$. japonicus and $S$. nasutus. The latter study also noticed high intraspecific genetic distances in S. japonicus when including data taken from populations from Japan and Taiwan, but variations in the species are not yet supported through morphological evaluation, except for the black caudal marking near the base of the lower lobe in Taiwanese specimens that is absent in other material. Compagno et al. (2005b) also noticed variations in this species when examining material from the Philippines and considered it to be a separated species (Squalus sp. 2) due to differences with $S$. japonicus and $S$. nasutus. In contrast, Last et al. (2007b) considered it to be con-specific with $S$. nasutus as the morphological differences pointed in Compagno et al. (2005b) and in their study represent intraspecific variations. Whether the Philippine form represents an undescribed species is still unclear, but we consider it here to be con-specific with $S$. nasutus for nomenclature stability.

Comparative material. Squalus nasutus. AMS I22817008, juvenile male, $362 \mathrm{~mm}$ TL, Northwest Shelf, 240 $\mathrm{km}$ north of Port Hedland, Western Australia, $18^{\circ} 06^{\prime} \mathrm{S}$, $117^{\circ} 45^{\prime} \mathrm{E}, 492-520 \mathrm{~m}$ depth; CSIRO H 4132-02, adult male, $465 \mathrm{~mm}$ TL, Bolinao evening market, Philippines; CSIRO H 4132-03, juvenile female, $475 \mathrm{~mm}$ TL, locality same as CSIRO H 4132-02; CSIRO H 4132-04, adult female, $540 \mathrm{~mm}$ TL, locality same as CSIRO H 4132-02; CSIRO H 5860-01, adult female, $540 \mathrm{~mm}$ TL, Cilacap (South coast, central Java), Indonesia, $07^{\circ} 40^{\prime} \mathrm{S}, 109^{\circ} 00^{\prime} \mathrm{E}$; CSIRO H 5860-02, adult female, $570 \mathrm{~mm}$ TL, locality same as CSIRO H 5860-01; CSIRO H 5860-03, adult female, $545 \mathrm{~mm}$ TL, locality same as CSIRO H 586001; CSIRO H 6125-04, adult male, 465 mm TL, Kedonganan, Jimbaran Bay (South coast of Bali), Indonesia, $08^{\circ} 45^{\prime} \mathrm{S}, 115^{\circ} 10^{\prime} \mathrm{E}$; CSIRO H 6413-01, adult female, 580 $\mathrm{mm}$ TL, West of Shark Bay, Western Australia, $25^{\circ} 03.8^{\prime} \mathrm{S}$, $112^{\circ} 08.9^{\prime} \mathrm{E}, 315-340 \mathrm{~m}$ depth; CSIRO H 6484-01, adult female, $575 \mathrm{~mm}$ TL, locality same as CSIRO H 586001. Type material of $S$. nasutus: CSIRO H2590-12, adult female, $503 \mathrm{~mm}$ TL, west of Leander Point, Western Australia, $29^{\circ} 15^{\prime} \mathrm{S}, 113^{\circ} 56^{\prime} \mathrm{E}$ (holotype); Paratypes: CSIRO H 2608-15, juvenile female, $411 \mathrm{~mm}$ TL, Rottnest Canyon, Western Australia, $31^{\circ} 57^{\prime} \mathrm{S}, 115^{\circ} 08^{\prime} \mathrm{E}$; CSIRO H 2598-07, juvenile female, $465 \mathrm{~mm}$ TL, west of Green Head, Western Australia, $29^{\circ} 58^{\prime} \mathrm{S}, 114^{\circ} 27^{\prime} \mathrm{E}$; CSIRO $\mathrm{H}$ 2567-08, adult male, $470 \mathrm{~mm}$ TL, west of Dorre Island, Western Australia, $25^{\circ} 09^{\prime} \mathrm{S}, 112^{\circ} 09^{\prime} \mathrm{E}$; CSIRO H 1652-01, juvenile female, $315 \mathrm{~mm}$ TL, northwest of Port Hedland, Western Australia, $18^{\circ} 25^{\prime} \mathrm{S}, 117^{\circ} 48^{\prime} \mathrm{E}$; CSIRO H 1652-02, juvenile female, $459 \mathrm{~mm}$ TL, same locality as CSIRO H 1652-01; CSIRO H 1207-07, adult female, 537 mm TL, northwest of Port Hedland, Western Australia, $18^{\circ} 20^{\prime} \mathrm{S}, 117^{\circ} 50^{\prime} \mathrm{E}$; CSIRO H 1207-08, juvenile female, 496 mm TL, same locality as CSIRO H 1207-07; CSIRO CA 3290, adult female, $549 \mathrm{~mm}$ TL, southwest of Rowley Shoals, Western Australia, $18^{\circ} 10^{\prime} \mathrm{S}, 118^{\circ} 20^{\prime} \mathrm{E}$; CSIRO CA 4055, adult female, $527 \mathrm{~mm}$ TL, southwest of Row- 
ley Shoals, Western Australia, $18^{\circ} 11^{\prime} \mathrm{S}, 118^{\circ} 04^{\prime} \mathrm{E}$; CSIRO H 2898-07, juvenile male, $452 \mathrm{~mm}$ TL, north-northwest of Port Hedland, Western Australia, $18^{\circ} 07^{\prime} \mathrm{S}, 118^{\circ} 12^{\prime} \mathrm{E}$; CSIRO CA 4110, adult male, $497 \mathrm{~mm}$ TL, east of Rowley Shoals, Western Australia, $17^{\circ} 18^{\prime} \mathrm{S}, 120^{\circ} 09^{\prime} \mathrm{E}$; CSIRO H 1693-01, juvenile male, $361 \mathrm{~mm}$ TL, Rowley Shoals, Western Australia, $17^{\circ} 02^{\prime} \mathrm{S}, 120^{\circ} 05^{\prime} \mathrm{E}$; CSIRO H $1693-$ 02, juvenile female, $306 \mathrm{~mm}$ TL, same locality as CSIRO H 1693-01; CSIRO H 1694-01, juvenile female, 425 mm TL, Rowley Shoals, Western Australia, 16 $6^{\circ} 7^{\prime} \mathrm{S}$, $120^{\circ} 14^{\prime} \mathrm{E}$; CSIRO H 2032-01, adult male, $461 \mathrm{~mm}$ TL, northeast of Mermaid Reef, Rowley Shoals, Western Australia, $16^{\circ} 54^{\prime} \mathrm{S}, 120^{\circ} 25^{\prime} \mathrm{E}$; CSIRO H 2032-02, juvenile female, $404 \mathrm{~mm}$ TL, same locality as CSIRO H 2032-01; WAM P 28086-006, juvenile male, 380 mm TL; juvenile female, $450 \mathrm{~mm}$ TL, Rowley Shoals, Western Australia, $17^{\circ} 49^{\prime} \mathrm{S}, 118^{\circ} 41^{\prime} \mathrm{E}, 308-310 \mathrm{~m}$ depth.

\section{Squalus brevirostris Tanaka, 1917}

Figs 3G, H, 5, 7I, J, 8D; Tables 4, 6

Japanese short-snout spurdog; Tsumari-aizam (Japanese)

Squalus brevirostris Tanaka 1917: 464-467, plates CXXIX and CXXX, figs 362-364 (original description, illustrated; type by original designation; type locality: Tokyo fish market, Japan, probably from Shimonoseki, Yamagueh Prefecture); Garrick 1960: 537 (cited; North-western Pacific Ocean); Zhu 1960: 106, 110-112 (description; Northwest Pacific Ocean); Chen et al. 1979: 26, 40 (cited; North-western Pacific Ocean); Zhu et al. 1984: 296 (cited; North-western Pacific Ocean); Siming et al. 1988: 1 (listed; Northwest Pacific Ocean); Nakabo 2002: 155 (listed; Ryukyu Islands, Japan); Duffy and Last 2007a: 31, 36, 37 (cited, North and Central Western Pacific Ocean); Ward et al. 2007: 116 (cited); Naylor et al. 2012: 58 (cited; North-western Pacific Ocean); Nakabo 2013: 195 (listed; Japan); Shinohara et al. 2014: 233 (listed; North-western Pacific Ocean).

Squalus megalops: Compagno 1984: 118-119 (cited; North-western Pacific Ocean); Zhu and Meng 2001: 311, 318-319 (cited, description; Northwest Pacific Ocean).

Type material. ZUMT 7630 (holotype), adult male, 426 mm TL, collected at Tokyo fish market, Japan, unknown collecting date and collector.

Additional material. CAS 15916, juvenile male, 380 $\mathrm{mm}$ TL, Formosa Strait, Taiwan, $26^{\circ} \mathrm{N}, 121^{\circ} \mathrm{E}, 90 \mathrm{~m}$ depth; CAS 30563, juvenile female, $270 \mathrm{~mm}$ TL, Formosa banks, Taiwan strait, Taiwan, $22^{\circ} 40^{\prime} \mathrm{N}, 118^{\circ} 30^{\prime} \mathrm{E}, 50$ m depth; CSIRO H 6293-29, juvenile female, 404 mm TL, Tashi fish market, near I-Lan (NE coast), Taiwan; CSIRO H 6483-02, adult male, 450 mm TL, off Kasasa, Kagoshima, Japan, East China Sea, $31^{\circ} 29^{\prime} \mathrm{N}, 130^{\circ} 02^{\prime} \mathrm{E}$, 145-150 m depth; HUMZ 33679, juvenile male, 390 mm TL, East China Sea, $29^{\circ} 54^{\prime} \mathrm{N}, 126^{\circ} 08^{\prime} \mathrm{E}$; HUMZ 95065 , adult female, $465 \mathrm{~mm}$ TL, East China Sea, $26^{\circ} 14.7^{\prime} \mathrm{N}$,

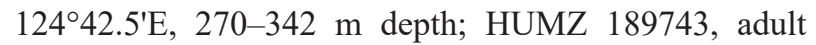
male, 387 mm TL, East China Sea; HUMZ 189745, adult female, 450 mm TL, East China Sea; HUMZ 189747 , adult male, 365 mm TL, East China Sea; HUMZ 189751, adult male, 395 mm TL, East China Sea; HUMZ 189756, adult female, $402 \mathrm{~mm}$ TL, East China Sea; HUMZ 189757, juvenile female, $357 \mathrm{~mm}$ TL, East China Sea; HUMZ 189758, adult female, $475 \mathrm{~mm}$ TL, East China Sea; HUMZ 189761, adult male, 400 mm TL, East China Sea; HUMZ 189762, adult male, 403 mm TL, East China Sea; HUMZ 189763, adult female, 433 mm TL, East China Sea; HUMZ 189767, juvenile male, 275 mm TL, East China Sea; KAUM-I 185, adult female, $500 \mathrm{~mm}$ TL, off Kasasa, Kagoshima, Japan, East China Sea, $31^{\circ} 29^{\prime} \mathrm{N}$, $130^{\circ} 02^{\prime} \mathrm{E}, 145-150 \mathrm{~m}$ depth; KAUM-I 187, adult female, $578 \mathrm{~mm}$ TL, locality same as KAUM-I 185; KAUM-I 377, adult male, $377 \mathrm{~mm}$ TL, locality same as KAUM-I 185; NSMT-P 47378, adult female, $600 \mathrm{~mm}$ TL, Central Pacific Ocean; NSMT P-47379, adult male, 452 mm TL, Derwent-Hunter Guyot, $30^{\circ} 36.3^{\prime} \mathrm{S}, 1^{\circ} 6^{\circ} 12.2^{\prime} \mathrm{E}$; NSMT-P 64979, two juvenile females, 480-520 mm TL, East China Sea, $29^{\circ} 0.57^{\prime} \mathrm{N}, 127^{\circ} 0.4^{\prime} \mathrm{E}, 167-170 \mathrm{~m}$ depth; SU 13468, adult female, $530 \mathrm{~mm}$ TL, Osaka market, Japan, 34.70N, 135.49E; SU 230373, neonate female, $270 \mathrm{~mm}$ TL, two neonate males, $200 \mathrm{~mm}$ TL, South China Sea, $19^{\circ} 6^{\prime} 30^{\prime \prime N}, 112^{\circ} 38^{\prime} 00^{\prime \prime E}, 111-119 \mathrm{~m}$ depth; ZUMT uncatalogued, female embryo, $147 \mathrm{~mm}$ TL, male embryo, $182 \mathrm{~mm}$ TL, Japan.

Diagnosis. A small-sized (373-578 mm TL in adults) Squalus species that differs from its Japanese congeners by: snout conspicuously short, its prenarial length $4.0 \%$, $3.8 \%-4.3 \%$ TL (vs. $5.4 \%-5.6 \%$ TL for $S$. shiraii sp. nov. vs. $4.1 \%-5.6 \%$ TL for $S$. mitsukurii vs. $5.9 \%-6.8 \%$ TL for S. japonicus vs. $4.7 \%-5.1 \%$ TL for $S$. acutirostris); dermal denticles unicuspid and lanceolate; pectoral-fin free rear tips conspicuously pointed; more elongate pectoral fins with its inner margin length $10.3 \%, 9.8 \%-11.2 \%$ TL (vs. $7.3 \%-8.3 \%$ TL for $S$. shiraii sp. nov. vs. $7.7 \%-9.5 \%$ TL for $S$. mitsukurii vs. $7.5 \%-8.8 \%$ TL for S. japonicus vs. $7.3 \%-8.7 \%$ TL for $S$. suckleyi vs. $6.6 \%-8.3 \%$ TL for S. acutirostris). It is further separated from S. japonicus and $S$. shiraii sp. nov. by narrower internarial space $(3.6 \%, 3.4 \%-3.8 \%$ TL vs. $4.1 \%, 4.1 \%-4.7 \%$ TL for $S$. japonicus vs. $4.8 \%, 4.1 \%-4.8 \%$ TL for $S$. shiraii sp. nov.) and from $S$. shiraii sp. nov. by larger second dorsal and pelvic fins (second dorsal-fin inner margin length 5.0\%, $4.8 \%-5.7 \%$ TL and pelvic fin length $11.7 \%, 10.9 \%$ $13.6 \%$ TL vs. $3.6 \%, 3.2 \%-4.1 \%$ TL and $10.7 \%, 9.5 \%$ $10.7 \%$ TL for S. shiraii sp. nov.) and wider caudal fin, its width at caudal fork $6.6 \%, 6.6 \%-7.3 \%$ TL (vs. $6.4 \%$, $5.7 \%-6.4 \%$ TL for $S$. shiraii sp. nov.). It is distinct from $S$. japonicus by snout rounded at tip, shorter preorbital $(6.7 \%, 6.5 \%-7.5 \%$ TL vs. $8.7 \%, 8.7 \%-10.3 \%$ TL for S. japonicus) and preoral length $(9.1 \%, 8.4 \%-9.5 \%$ TL vs. $10.7 \%, 10.7 \%-12.2 \%$ TL for $S$. japonicus). Squalus brevirostris is easily separated from $S$. mitsukurii and $S$. shiraii sp. nov. by mononspondylous and total vertebrae (40, 37-42 and $110,105-114$ vs. 44-46 and 116-117 for S. mitsukurii vs. 47, 44-48 and 122, 120-123 for S. shiraii $\mathrm{sp}$. nov.). It also exhibits lower number of precaudal 

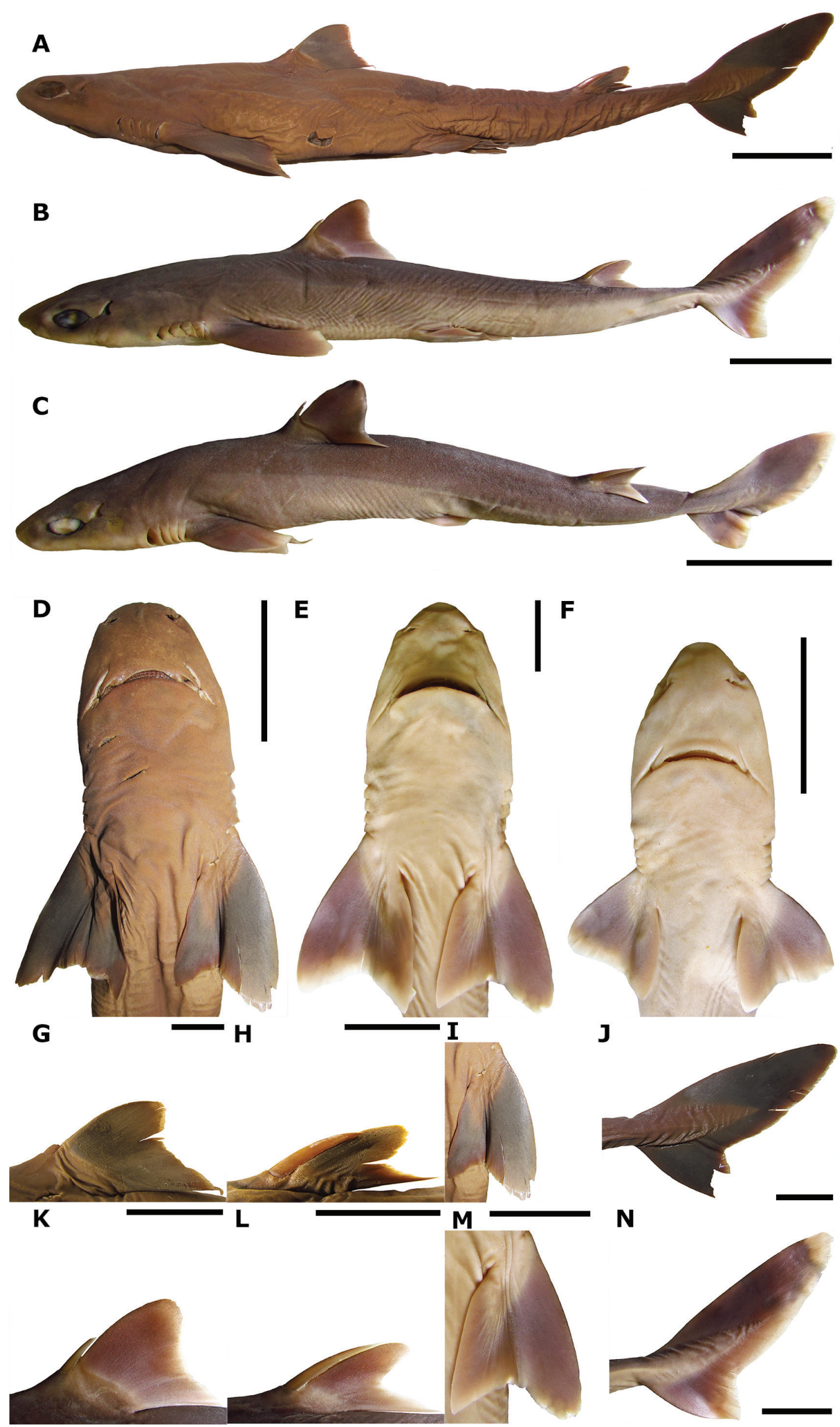

Figure 5. Squalus brevirostris: lateral $(\mathbf{A}-\mathbf{C})$ and ventral $(\mathbf{D}-\mathbf{F})$ views; first $(\mathbf{G}, \mathbf{K})$ and second $(\mathbf{H}, \mathbf{L})$ dorsal fins; pectoral (I, M) and caudal (J,N) fins. ZUMT 7630 (holotype), adult male, 426 mm TL (A, D, G-J); HUMZ 189762, adult male, 403 mm TL (B, E, K-N); HUMZ 189767, juvenile male, 275 mm TL (C, F). Scale bars: $50 \mathrm{~mm}(\mathbf{A}-\mathbf{F}) ; 20 \mathrm{~mm}(\mathbf{G}-\mathbf{N})$. 
vertebrae than $S$. shiraii sp. nov. (83, 78-89 vs. 93, 91-94 for $S$. shiraii sp. nov.).

Description. External morphology. Small size species (147.0-578.0 mm TL) of Japanese dogfish. Body fusiform and slender, equally deep from head to trunk (head height $0.9,0.9-1.0$ times trunk height and 1.0, 0.8-1.2 times abdomen height) (Fig. 5A-C). Head very short, its length $21.9 \%(20.0 \%-29.6 \%)$ TL; head width $1.4(1.0-$ 1.4) times broader than trunk width and 1.1 (1.1-1.6) times abdomen width. Snout somewhat obtuse ventrally, very short (preorbital length $6.7 \%, 6.5 \%-7.5 \%$ TL); anterior nasal flap broad and bifurcated, nearer to snout tip than to mouth with prenarial length $0.8(0.8-0.9)$ times distance from nostril to upper labial furrow (Fig. 5D-F). Eyes oval with anterior margin rounded and posterior margin notched; eyes large, its length 2.3 (1.8-2.5) times its height. Prespiracular length $0.6(0.5-0.6)$ times prepectoral length and $1.8(1.6-1.9)$ times preorbital length. Spiracle crescent and narrow, its length $0.3(0.3-$ 0.4 ) times eye length, nearer to posterior margin of the eye than to first gill slit. First and fifth gill slits somewhat concave and low; fifth gill slit $1.1(0.9-1.6)$ times higher than first gill slit.

Preoral length somewhat equal to mouth width, corresponding to $1.1(1.1-1.2)$ times the latter. Mouth markedly arched and narrow, its width 2.3 (1.8-2.3) times broader than internarial space; upper labial furrow small, its length $2.6 \%(2.0 \%-2.7 \%$ TL) with thin fold; lower labial furrow also short with fold subdivided into three small inter-digits. Teeth tiny and unicuspid, labial-lingually flattened and alternate, similar in both jaws, lower teeth slightly wider than the upper teeth; cusp short and oblique; mesial cutting edge convex; both distal and mesial heels rounded; apron elongate in upper and lower teeth (Fig. 3G, H). Three and two series of functional teeth on upper and lower jaws for holotype (two series for non-type specimens); 12-0-13 (12-0-12) teeth rows in upper jaw; $10-0-10(10-0-10)$ teeth rows in lower jaw.

Pre-first dorsal length 1.4 (1.3-1.6) times prepectoral length. First dorsal fin short, its length 1.7 (1.5-1.9) times its height; first dorsal-fin low, its height 1.3 (1.2-1.5) times greater than its inner margin length; first dorsal-fin anterior margin concave and posterior margin straight; first dorsal-fin free, rear tip pointed; first dorsal-fin apex rounded and slender at fin web (Fig. 5G, K). Origin of first dorsal-fin spine anterior to vertical traced at free rear tips of pectoral fins; first dorsal-fin spine short (broken in the holotype), never reaching first dorsal-fin apex, its length 0.3-0.6 times first dorsal-fin height. Interdorsal space 1.1 (1.1-1.3) times prepectoral length and 2.4 (2.2-2.6) times larger than dorsal-caudal space. Pre-second dorsal length 2.8 (2.7-3.3) times greater than prepectoral length and 3.0 (2.8-3.2) times the length of dorsal caudal margin. Second dorsal fin very short, its length $0.9(0.8-1.0)$ times first dorsal-fin length; second dorsal fin low, its height 1.2 (0.8-1.4) times length of second dorsal-fin inner margin; second dorsal-fin anterior margin concave and posterior margin markedly falcate; second dorsal-fin apex rounded and evidently slender at fin web (Fig. 5H, L). Second dorsal-fin spine thick and elongate, although not reaching the apex, its length $0.8(0.8-1.1)$ times the fin height; second dorsal-fin spine length 1.3-2.6 times greater than length of first dorsal-fin spine.

Pectoral fin conspicuously narrow with pectoral-fin anterior margin length 1.4 (1.3-1.9) times larger than pectoral-fin posterior margin length and 1.4 (1.1-1.5) times larger than pectoral-fin inner margin length; pectoral-fin anterior and inner margins convex; pectoral-fin posterior margin conspicuously concave; pectoral-fin apex rounded and lobe-like; pectoral-fin free rear tips markedly pointed and triangular, markedly lobe-like (Fig. 5I, M); pectoral-fin free rear tips reaching the horizontal line traced at pectoral-fin apex. Pectoral-pelvic space $0.8(0.6-0.9)$ times pelvic-caudal space. Pelvic fins nearer to first dorsal fin than second dorsal fin. Pelvic fins very narrow with pelvic-fin margins straight; pelvic-fin apex rounded; pelvic-fin free rear tips triangular, pointed and lobe-like; pelvic fin length $1.2(0.9-1.2)$ times length of preventral caudal margin. Clasper flattened ventrally and small, its outer length $3.9 \%(1.4 \%-5.1 \% \mathrm{TL})$, transcending the pelvic-fin free rear tips; clasper groove dorsal, longitudinal and short; apopyle and hypopyle with conspicuous apertures, near each other; rhipidion bladelike, located at the posterior end of the clasper; inner length of clasper $1.2(0.5-1.2)$ times length of pelvic-fin inner margin.

Caudal keel prominent, reaching from forward second dorsal fin insertion to behind origin of caudal fin. Caudal fin slightly slender in the dorsal caudal lobe; dorsal caudal and upper postventral caudal margins convex; dorsal caudal tip rounded (Fig. 5J, N); dorsal caudal margin $0.9(0.7-1.0)$ times head length and $2.0(1.5-2.0)$ times larger than preventral caudal margin; lower postventral caudal margin convex (broken in holotype); preventral caudal margin strongly convex and short, its length 1.7 (1.6-2.3) times greater than length of pelvic-fin inner margin; ventral caudal tip rounded (broken in holotype); caudal fork discontinuous between lobes, its width $6.6 \%$ $(6.6 \%-7.6 \%)$ TL.

Squamation (Fig. 7I, J). Dermal denticles unicuspid and lanceolate, not imbricated; cusp rounded and posterior; denticles conspicuously slender at crown, although markedly broad medial-anteriorly at its base; denticles with length much greater than its width; median ridge tall and thick, bifurcated anteriorly with profound anterior furrow; lateral ridges tall and thin, shorter than median ridge.

Colouration (Fig. 5). Body brownish-grey dorsally, white ventrally and latero-posteriorly Pectoral fins grey with pectoral-fin posterior and inner margins white. Pelvic fins white dorsally and ventrally. First dorsal fin brownish-grey, slightly white at first dorsal-fin posterior margin, free rear tip and fin base; second dorsal fin light brown, whitish at the fin base, somewhat white at second dorsal-fin apex, posterior margin and freer rear tip. Dorsal-fin spines grey, dark grey anteriorly and white at 
Table 4. External measurements expressed as percentage of total length (\% TL) for the holotype (ZUMT 7630) and other specimens of $S$. brevirostris. TL is expressed in millimeters. N: number of specimens; $x$ : mean; SD: standard deviation.

\begin{tabular}{|c|c|c|c|c|c|}
\hline & Holotype & $\mathbf{N}$ & & $x$ & SD \\
\hline Total length (mm) & 426.0 & 14 & $147.0-578.0$ & 408.2 & 100.9 \\
\hline Precaudal length & 79.8 & 14 & $75.1-81.3$ & 79.2 & 1.3 \\
\hline Pre-second dorsal length & 61.7 & 14 & $58.4-63.8$ & 61.9 & 1.3 \\
\hline Pre-first dorsal length & 29.6 & 14 & $28.5-31.7$ & 30.0 & 1.0 \\
\hline Pre-vent length & 47.7 & 14 & $43.1-49.5$ & 46.5 & 1.6 \\
\hline Prepelvic length & 43.7 & 14 & $42.4-46.9$ & 44.1 & 1.3 \\
\hline Prepectoral length & 21.7 & 14 & 19.0-22.9 & 21.2 & 1.0 \\
\hline Head length & 21.9 & 14 & $20.0-29.6$ & 22.5 & 2.2 \\
\hline Prebranchial length & 17.9 & 14 & $16.8-21.0$ & 18.3 & 0.9 \\
\hline Prespiracular length & 12.1 & 14 & $11.3-12.9$ & 12.1 & 0.5 \\
\hline Preorbital length & 6.7 & 14 & $6.5-7.5$ & 7.1 & 0.3 \\
\hline Prenarial length & 4.0 & 14 & $3.8-4.4$ & 4.1 & 0.2 \\
\hline Preoral length & 9.1 & 14 & $8.4-10.0$ & 9.1 & 0.5 \\
\hline Inner nostril-labial furrow space & 5.0 & 14 & $4.5-5.5$ & 4.9 & 0.3 \\
\hline Mouth width & 8.1 & 14 & $7.4-8.6$ & 7.9 & 0.3 \\
\hline Labial furrow length & 2.6 & 14 & $2.0-2.7$ & 2.4 & 0.2 \\
\hline Internarial space & 3.6 & 14 & $3.4-4.7$ & 3.7 & 0.3 \\
\hline Interorbital space & 8.6 & 14 & $7.8-10.3$ & 8.4 & 0.6 \\
\hline Eye length & 5.0 & 14 & $4.2-6.1$ & 4.7 & 0.5 \\
\hline Eye height & 2.2 & 14 & $1.8-2.6$ & 2.2 & 0.2 \\
\hline Spiracle length & 1.7 & 14 & $1.4-2.4$ & 1.7 & 0.3 \\
\hline First gill-slit height & 2.1 & 14 & $1.5-2.6$ & 2.0 & 0.3 \\
\hline Fifth gill-slit height & 2.3 & 14 & $1.7-2.6$ & 2.3 & 0.2 \\
\hline Interdorsal space & 24.9 & 14 & $23.3-27.2$ & 24.7 & 1.2 \\
\hline Dorsal-caudal space & 10.5 & 14 & $9.9-10.6$ & 10.3 & 0.2 \\
\hline Pectoral-pelvic space & 21.4 & 14 & $17.2-22.8$ & 20.0 & 1.9 \\
\hline Pelvic-caudal space & 28.1 & 14 & $24.6-29.4$ & 27.5 & 1.3 \\
\hline First dorsal-fin length & 13.0 & 14 & $12.6-14.7$ & 13.7 & 0.5 \\
\hline First dorsal-fin anterior margin & 10.3 & 14 & $7.9-12.0$ & 10.9 & 1.0 \\
\hline First dorsal-fin base length & 7.3 & 14 & $7.4-9.2$ & 7.8 & 0.5 \\
\hline First dorsal-fin height & 7.6 & 14 & $6.5-8.6$ & 7.9 & 0.5 \\
\hline First dorsal-fin inner margin & 5.8 & 14 & $5.2-6.8$ & 6.0 & 0.4 \\
\hline First dorsal-fin posterior margin & 7.7 & 14 & $6.6-9.6$ & 8.6 & 0.7 \\
\hline First dorsal-fin spine length & - & 12 & $1.6-4.2$ & 3.3 & 0.7 \\
\hline First dorsal-fin spine base width & 0.7 & 14 & $0.5-0.8$ & 0.6 & 0.1 \\
\hline Second dorsal-fin length & 11.6 & 14 & $11.2-13.2$ & 12.3 & 0.7 \\
\hline Second dorsal-fin anterior margin & 9.8 & 14 & $9.7-12.4$ & 10.8 & 0.7 \\
\hline Second dorsal-fin base length & 6.6 & 14 & $6.1-7.9$ & 7.1 & 0.6 \\
\hline Second dorsal-fin height & 6.0 & 14 & $5.2-7.1$ & 6.3 & 0.6 \\
\hline Second dorsal-fin inner margin & 5.0 & 14 & $4.8-6.7$ & 5.4 & 0.5 \\
\hline Second dorsal-fin posterior margin & 4.3 & 14 & $2.9-5.3$ & 4.7 & 0.6 \\
\hline Second dorsal-fin spine length & 4.6 & 14 & $4.2-6.6$ & 5.4 & 0.7 \\
\hline Second dorsal-fin spine base width & 0.8 & 14 & $0.7-0.9$ & 0.8 & 0.1 \\
\hline Pectoral-fin anterior margin length & 14.7 & 14 & $12.0-15.7$ & 14.3 & 0.9 \\
\hline Pectoral-fin inner margin length & 10.3 & 14 & $9.8-12.0$ & 10.6 & 0.6 \\
\hline Pectoral-fin base length & 4.7 & 14 & $3.8-5.3$ & 4.5 & 0.5 \\
\hline Pectoral-fin posterior margin length & 10.4 & 14 & $6.3-11.5$ & 10.4 & 1.2 \\
\hline Pelvic length & 11.7 & 14 & $10.3-13.6$ & 11.7 & 0.7 \\
\hline Pelvic-fin inner margin length & 6.1 & 14 & $4.8-6.7$ & 6.0 & 0.5 \\
\hline Dorsal caudal margin length & 20.5 & 14 & $19.2-21.5$ & 20.5 & 0.5 \\
\hline Preventral caudal margin length & 10.1 & 14 & $10.1-12.4$ & 10.9 & 0.6 \\
\hline Caudal fork width & 6.6 & 14 & $6.6-7.6$ & 7.0 & 0.3 \\
\hline Head width at nostrils & 7.7 & 14 & $6.2-9.1$ & 7.0 & 0.7 \\
\hline Head width at mouth & 12.1 & 14 & $10.7-12.7$ & 11.6 & 0.5 \\
\hline Head width & 12.1 & 14 & $11.0-14.1$ & 12.7 & 0.8 \\
\hline Trunk width & 8.9 & 14 & $8.0-12.9$ & 10.3 & 1.5 \\
\hline Abdomen width & 11.2 & 14 & $7.1-12.4$ & 9.7 & 1.6 \\
\hline Head height & 9.9 & 14 & $9.0-11.9$ & 10.0 & 0.8 \\
\hline Trunk height & 10.7 & 14 & $9.3-13.1$ & 10.5 & 1.3 \\
\hline Abdomen height & 10.1 & 14 & $7.7-13.2$ & 10.5 & 1.7 \\
\hline Clasper outer length & 3.9 & 6 & $1.4-5.1$ & 4.1 & 1.3 \\
\hline Clasper inner length & 7.1 & 6 & $3.0-8.1$ & 6.8 & 1.7 \\
\hline
\end{tabular}


the tip. Caudal fin light brown, whitish near vertebral column; postventral caudal margins white, broadly white at dorsal caudal tip and from ventral caudal tip to caudal fork; light black caudal stripe.

Vertebral counts (Table 6). 83 (holotype) 78-89 (other material) precaudal vertebrae; 70 (66-72) diplospondylous vertebrae; 110 (105-114) total vertebrae.

Geographical distribution. This species occurs in tropical and subtropical areas of the North-western Pacific Ocean from Southern Japan, China and Taiwan on continental shelves and upper continental slopes between 50-342 m depth (Fig. 8D). It is also recorded from the Philippines in Manjaji-Matsumoto (2017).

Remarks. Morphological variations in the shape of pectoral and dorsal fins, dermal denticles and colour of caudal fin are observed within S. brevirostris. First dorsal-fin posterior margin is somewhat straight near its apex like in the holotype, although other specimens exhibit first dorsal-fin posterior margin concave. Pectoral fins are slightly broad at posterior margin in specimens from the Central Western Pacific Ocean (except Taiwan), while it is narrow in others like that observed for the holotype. Pelvic fins are nearer to first dorsal fin, although it may be in the midline between the two dorsal fins in a few specimens. Caudal fin has postventral caudal margins white, although narrowly white at caudal fork in specimens from Kagoshima. Thickness of the dermal denticles varies from very slender to conspicuously broad at the crown in few specimens from Kagoshima. These variations are possibly not related to dimorphism or ontogeny and apparently it is scattered, as it has an unclear morphological pattern throughout the distributional range of this species in the North and Central Western Pacific Ocean. Duffy and Last (2007a) previously noticed differences in the dermal denticles, which was suggested to be due to ontogeny. Further comparative investigations are required to include more specimens from Taiwan and South Korea in order to better understand these variations within the species.

Squalus brevirostris exhibits low vertebral counts that are usually overlapped to those of morphologically similar species occurring elsewhere, such as $S$. megalops, $S$. acutipinnis and $S$. lobularis. However, it can be separated from other similar species by precaudal vertebrae with $S$. hemipinnis, S. notocaudatus and S. formosus, total vertebrae with $S$. hemipinnis, $S$. notocaudatus, S. formosus and $S$. albifrons and monospondylous vertebrae with $S$. notocaudatus, S. formosus, S. albifrons and S. bucephalus.

\section{Squalus acutirostris Zhu, Meng \& Li, 1984}

Figs 6A-E, 8E; Table 2

Shortspine spurdog

Squalus acutirostris Zhu, Meng and Li 1984: 283-286, fig.1 (original description; illustrated; type by original designation; type locality: South China Sea, $18^{\circ} 51^{\prime}-18^{\circ} 47^{\prime} \mathrm{N}, 112^{\circ} 41^{\prime}-112^{\circ} 33^{\prime} \mathrm{E}, 394 \mathrm{~m}$ depth).
Squalus mitsukurii: White and Last 2013: 229-230 (cited, illustrated; Taiwan); Motomura et al. 2015: 12-13 (cited, listed, illustrated; Uji Islands, East China Sea); Weigmann 2016: 904 (cited; Western Pacific Ocean).

S. japonicus: Quang et al. 2013: 22, 27-28 (cited, listed, illustrated; Quy Nhon Bay, Vietnam).

Type material. SCSFRI D01562 (holotype), adult male, $635 \mathrm{~mm}$ TL, South China Sea, $18^{\circ} 51^{\prime}-18^{\circ} 47^{\prime} \mathrm{N}, 112^{\circ} 41^{\prime}-$ $112^{\circ} 33^{\prime} \mathrm{E}, 394 \mathrm{~m}$ depth, collected on 21 April 1982; SCSFRI D01548 (paratype), adult female, $975 \mathrm{~mm}$ TL, South China Sea, $18^{\circ} 50.8^{\prime}-19^{\circ} 28.8^{\prime} \mathrm{N}, 112^{\circ} 47.3^{\prime}-113^{\circ} 58.8^{\prime} \mathrm{E}$.

Additional material. HUMZ 74990, juvenile female, $536 \mathrm{~mm}$ TL, Kyushu-Palau Ridge, $26^{\circ} 14.1^{\prime}-26^{\circ} 10.1^{\prime} \mathrm{N}$, $135^{\circ} 47.5^{\prime}-135^{\circ} 48.0^{\prime} \mathrm{E}, 360 \mathrm{~m}$ depth; NMMB P 15619 (photo only), adult female, $700 \mathrm{~mm}$ TL, Pingtung county, Taiwan.

Diagnosis. Single values correspond to holotype first followed by a single paratype and a specimen from Japan between brackets. Squalus acutirostris is separated from its congeners by a combination of characters: large sized species (635-975 mm maximum TL in adults) with body moderately slender and fusiform (Fig. 6A), its greatest width at head (head width $1.4(1.4,1.2)$ times trunk width and $1.3(1.1,1.2)$ times abdomen width); head small, its length $24.4 \%(22.6 \%, 22.1 \%) \mathrm{TL}$; snout rounded at tip and short, preorbital length $8.2 \%(7.3 \%, 8.1 \%) \mathrm{TL}$; nostrils nearer to snout than to mouth (Fig. 6B); first dorsal fin oblique, wide at fin web and low, its height 6.0\%(5.6\%, $8.6 \%$ ) TL; first dorsal fin concave at the lower half of posterior margin, not raked (Fig. 6C); second dorsal fin with raked posterior margin (Fig. 6D); dorsal-fin spines robust and short, never reaching the fin apex (first dorsal-fin spine length $2.8 \%(2.7 \%, 3.2 \%) \mathrm{TL}$; second dorsal-fin spine length $2.8 \%(1.9 \%, 4.8 \%)$ TL; pectoral fins moderately wide with posterior margin length $11.0 \%(10.3 \%$, $10.1 \%) \mathrm{TL}$; pectoral-fin posterior margin concave, although not falcate; pectoral-fin free rear tips rounded; caudal fin rectangular with conspicuous caudal fork (Fig. $6 \mathrm{E}$ ); caudal fin small with dorsal caudal margin length $0.7(0.9,1.0)$ times head length; lower caudal lobe much shorter than upper caudal lobe (preventral caudal margin length $0.7(0.6,0.5)$ times dorsal caudal margin length); body brownish-grey to dark grey dorsally and light brown ventrally; first and second dorsal-fin apex slightly black; conspicuous black upper caudal blotch and small black caudal bar; black basal marking conspicuous at the origin of caudal fin; lower caudal lobe mostly white.

Geographical distribution. Squalus acutirostris occurs in the North-western Pacific Ocean with reports from Japan, Taiwan and China (Fig. 8E). It has been previously misidentified with $S$. japonicus in Vietnam as seen in Quang et al. (2013). It inhabits continental slopes between 360-525 $\mathrm{m}$ depth (Zhu et al. 1984; present study). 


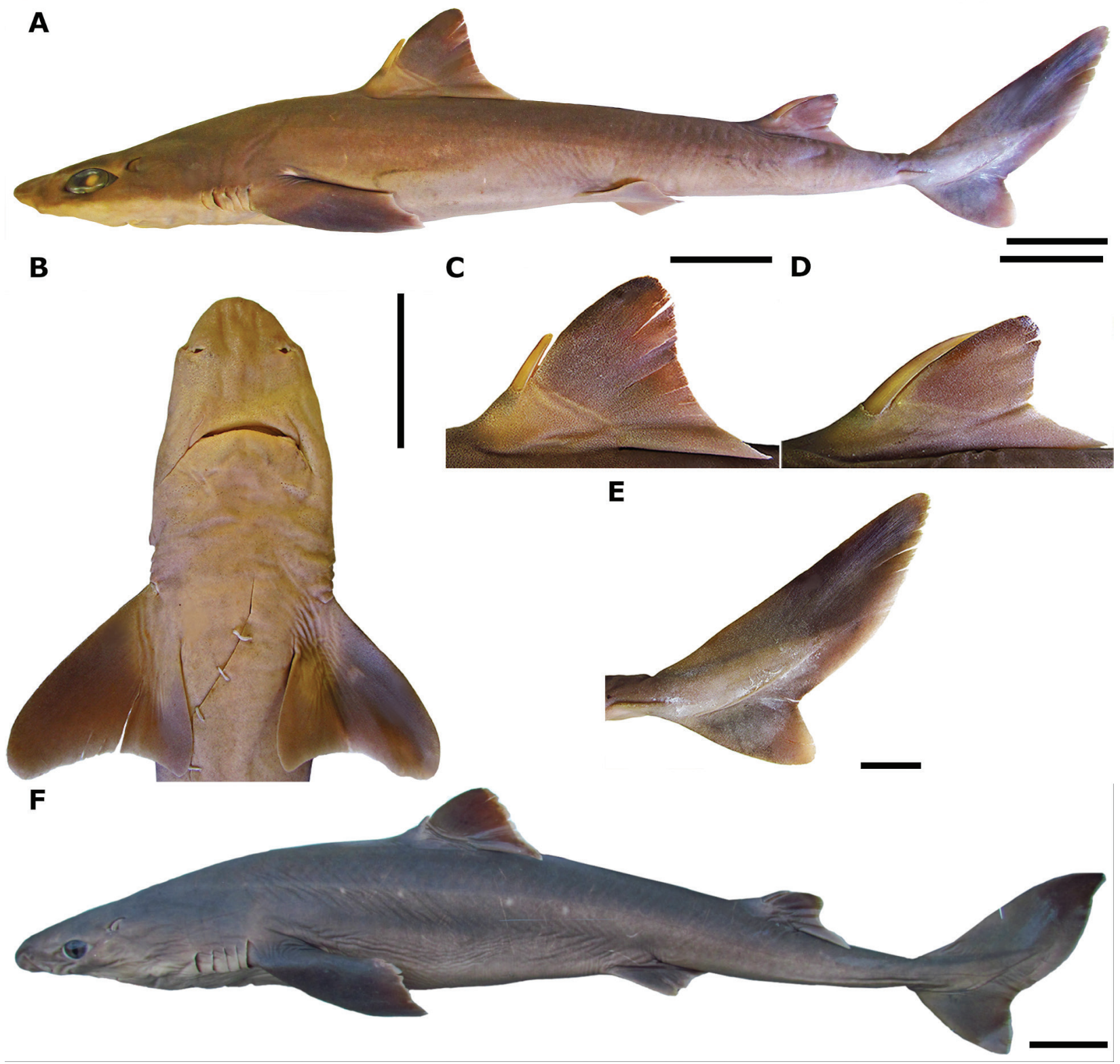

Figure 6. Squalus acutirostris (A-E): HUMZ 74990, juvenile female, $536 \mathrm{~mm}$ TL in lateral (A) and ventral (B) views; first (C) and second (D) dorsal fins and caudal fin (E). S. suckleyi in lateral view (F): HUMZ 90963, adult female, 784 mm TL. Scale bars: $50 \mathrm{~mm}(\mathbf{A}, \mathbf{B}, \mathbf{F}) ; 20 \mathrm{~mm}(\mathbf{C}-\mathbf{E})$.

Remarks. Squalus acutirostris, originally described from the South China Sea, was previously recognised in China and Western Australia in Zhu et al. (1984), Muñoz-Chápuli and Ramos (1989) and Zhu and Meng (2001). White and Last (2013) have proven that the Australian records correspond to a distinct species, S. grahami White, Last \& Stevens, 2007. Furthermore, occurrence of $S$. acutirostris in the South China Sea was thought to be related to $S$. mitsukurii (White and Last 2013; Weigmann 2016) due to no apparent morphological and meristic distinction and, thus, this species was designated as a junior synonym. According to White and Last (2013), the differences in external measurements (e.g. precaudal length, interdorsal space, dorsal caudal margin length) between these species were due to the preservation condition of the holotype of $S$. acutirostris. However, the present analysis reveals many morphometric differences when adults are compared, such as: inner nostril-labial furrow space $(6.9 \%-8.6 \% \mathrm{TL}$ vs. $3.9 \%-4.5 \%$ TL for $S$. mitsukurii); first dorsal fin height (5.6\%-6.0\% TL vs. $6.9 \%-9.8 \%$ TL for S. mitsukurii); second dorsal-fin anterior margin length $(6.8 \%-7.6 \%$ TL vs. $7.7 \%-10.2 \%$ TL for $S$. mitsukurii); second dorsal-fin base length $(4.8 \%-5.8 \%$ TL vs. $6.8 \%-7.9 \%$ TL for $S$. mitsukurii); second dorsal fin height (3.3\%-3.6\% TL vs. 4.5\%-6.8\% TL for S. mitsukurii); second dorsal-fin spine length and base width $(1.9 \%-2.8 \%$ TL, $0.5 \%-0.6 \%$ TL vs. $3.4 \%-5.3 \%$ TL, $0.7 \%-1.0 \%$ TL for $S$. mitsukurii). Further distinctive characteristics between $S$. mitsukurii and 
the types of $S$. acutirostris include conspicuously shorter snout with nostrils clearly nearer to the snout tip than to the mouth, its prenarial length $0.6-0.8$ times nostril-labial furrow space (vs. elongate snout with nostrils nearer to the mouth, its prenarial length 1.1-1.4 times nostril-labial furrow space in $S$. mitsukurii) and narrower pectoral and pelvic fins (vs. conspicuously broad pectoral and pelvic fins in $S$. mitsukurii). These characteristics are congruent with observations of Zhu et al. (1984) and Zhu and Meng (2001) and are apparent, regardless of the preservation condition and which support the distinction between $S$. mitsukurii and the nominal species $S$. acutirostris.

Squalus acutirostris may be separated from the Japanese congeners by having more elongate second dorsal-fin length $63.9 \%-64.0 \%$ TL (vs. 60.5\%-63.4\% TL for S. shiraii sp. nov. vs. $58.4 \%-63.9 \%$ TL for $S$. mitsukurii vs. $60.7 \%-62.0 \%$ TL for $S$. japonicus vs. $61.1 \%-63.8 \%$ TL for $S$. brevirostris vs. 56.2\%-62.3\% TL for S. suckleyi) and larger inner nostril-labial furrow space $6.9 \%-$ $8.6 \%$ TL (vs. $4.3 \%-4.9 \%$ TL for $S$. shiraii sp. nov. vs. $3.9 \%-4.5 \%$ TL for $S$. mitsukurii vs. $4.6 \%-5.1 \%$ TL for $S$. japonicus vs. $4.5 \%-5.1 \%$ TL for $S$. brevirostris vs. 3.9\%$5.6 \%$ TL for $S$. suckleyi). It may also be distinguished from $S$. shiraii sp. nov., S. japonicus and S. brevirostris by pre-pelvic length $47.2 \%-49.3 \%$ TL and prenarial length $4.7 \%-5.1 \%$ TL (vs. $44.1 \%-46.8 \%$ TL and $5.4 \%-5.6 \%$ TL for S. shiraii vs. $43.7 \%-45.9 \%$ TL and $5.9 \%-6.8 \%$ TL for $S$. japonicus vs. $42.4 \%-46.9 \%$ TL and $3.8 \%-4.3 \%$ TL for $S$. brevirostris). It is also easily separated from $S$. brevirostris by having pectoral free rear tips rounded (vs. conspicuously pointed).

\section{Squalus suckleyi (Girard, 1854)}

Figs 3I, J, 6F, 7K, L, 8F; Tables 5, 6

Spotted spiny dogfish; Northeast Pacific spiny dogfish

Acanthias vulgaris: Müller and Henle 1841: 83-84 (revision; North Pacific Ocean).

Spinax (Acanthias) suckleyi Girard 1854: 196 (original description; no types originally designated or known; Fort Steilacoom, Puget Sound, Washington State, The United States of America).

Squalus suckleyi Fowler 1941: 258-260 (description; North Pacific Ocean); Ebert et al. 2010: 22-40 (re-description, DNA barcoding, designation of neotype; North Pacific Ocean); Ward et al. 2007: 119 (cited; North Pacific Ocean); Naylor et al. 2012: 57 (DNA barcoding; North Pacific Ocean); Orlov et al. 2012: 1-12 (life history; North Pacific Ocean); Ebert et al. 2013: 78, 95 (cited, description; Northeast Pacific Ocean).

Squalus acanthias: Bigelow and Schroeder 1957: 30 (revision; Northwest Pacific Ocean); Jones and Geen 1976: 2500-2506 (taxonomy; North Pacific Ocean); Ward et al. 2007: 118-130 (DNA barcoding; North Pacific Ocean).

Squalus acanthias suckleyi: Myagkov and Kondyurin 1986: 1-18 (cited; North Pacific Ocean); Bass et al. 1976: 10 (cited; North Pacific Ocean).

Squalus wakiyae Tanaka 1917: 471-475, pl. 130, figs 368-370 (original description; illustrated; type by original designation from Watanoha, Japan; Northwest Pacific Ocean).
Squalus acanthias not Linnaeus: Zhu 1960: 107-109 (description; Northwest Pacific Ocean); Zhu and Meng 2001: 310-313 (description; Northwest Pacific Ocean); Nakabo 2002: 155 (listed; Japan); Nakabo 2013: 194 (listed; Japan); Shinohara et al. 2014: 233 (listed; Northwest Pacific Ocean); Dyldin 2015: 56-57 (listed; Northwest Pacific Ocean).

Type material. CAS 227267, adult male, $674 \mathrm{~mm}$ TL, Hood Canal, Puget Sound, Washington State, The United States of America, $47^{\circ} 22^{\prime} \mathrm{N}, 123^{\circ} 05^{\prime} \mathrm{E}, 30 \mathrm{~m}$ depth. Collected on 3 August 2007. Neotype designated in Ebert et al. (2010).

Additional material. HUMZ 75718, juvenile female, 500 mm TL, Usujiri, Mimamikayakabie, Hokkaido, Japan; HUMZ 81094, adult female, 565 mm TL, Usujiri, Mimamikayakabie, Hokkaido, Japan; HUMZ 87733, juvenile male, $495 \mathrm{~mm}$ TL, off Shiretoko, Hokkaido, Japan; HUMZ 87752, juvenile female, 455 mm TL, off Muroran, Hokkaido, Japan; HUMZ 90963, adult female, $784 \mathrm{~mm}$ TL, Notori Misaki oki, Japan; HUMZ 107865, juvenile female, 465 mm TL, off Sekinai, Kumaishi, Hokkaido, Japan; HUMZ 107869, adult female, $650 \mathrm{~mm}$ TL, off Sekinai, Kumaishi, Hokkaido, Japan; HUMZ 123859, adult male, $815 \mathrm{~mm}$ TL, north Japan, $44^{\circ} 00.11^{\prime} \mathrm{N}, 155^{\circ} 00.1 \mathrm{E}$; NSMT-P 10540, adult female, $625 \mathrm{~mm}$ TL, Northern Japan, Japan; NSMT-P 42569, adult male, $597 \mathrm{~mm}$ TL, unknown locality; NSMT-P 61090, adult female, $915 \mathrm{~mm}$ TL, Northern Japan, Japan; NSMT-P 74887, juvenile male, 445 mm TL, Northern Japan, Japan; NSMT-P 77186, adult male, $835 \mathrm{~mm}$ TL, unknown locality; NSMT-P 79501, adult male, $740 \mathrm{~mm}$ TL, Northern Japan, Japan; NSMT-P 92640, adult female, 740 mm TL, Northern Japan, Japan; ZUMT 3231, neonate male, $272 \mathrm{~mm}$ TL, Nagasaki, Japan; ZUMT 4684, neonate male, $265 \mathrm{~mm}$ TL, Tokyo Fish Market, Tokyo, Japan; ZUMT 4685, neonate female, 270 mm TL, Tokyo Fish Market, Tokyo, Japan; ZUMT 10536, neonate female, $120 \mathrm{~mm}$ TL; ZUMT 10789, neonate female, 238 mm TL, Hokkaido, Japan; ZUMT 36806, neonate female, $202 \mathrm{~mm}$ TL, Sakhalin Island, Russia; ZUMT 36807, neonate female, $200 \mathrm{~mm}$ TL, Sakuharin, Japan; ZUMT 36825, neonate female, $202 \mathrm{~mm}$ TL, Sakhalin Island, Russia; ZUMT 36836, neonate female, $202 \mathrm{~mm}$ TL, Sakuharin, Japan; ZUMT 40117, neonate male, $257 \mathrm{~mm}$ TL, Uozu, Toyama Prefecture, Japan; ZUMT 41539, neonate male, $284 \mathrm{~mm}$ TL, Uozu, Toyama Prefecture, Japan; ZUMT 45801, neonate male, 270 mm TL, Uozu, Toyama Prefecture, Japan; ZUMT 46116, neonate male, $284 \mathrm{~mm}$ TL, Uozu, Toyama Prefecture, Japan; ZUMT 46123, neonate male, $254 \mathrm{~mm}$ TL, Uozu, Toyama Prefecture, Japan; ZUMT 46124, neonate male, 288 mm TL, Uozu, Toyama Prefecture, Japan; ZUMT 46151, neonate male, $272 \mathrm{~mm}$ TL, Uozu, Toyama Prefecture, Japan; ZUMT 46670, neonate male, $258 \mathrm{~mm}$ TL, Uozu, Toyama Prefecture, Japan; ZUMT 46671, neonate female, $250 \mathrm{~mm}$ TL, Uozu, Toyama Prefecture, Japan; ZUMT 46672, neonate male, 230 mm TL, Uozu, Toyama Prefecture, Japan; ZUMT 46673, neonate female, $260 \mathrm{~mm}$ TL, Uozu, Toyama Prefecture, 
Table 5. External measurements of S. suckleyi expressed as percentage of the total length (\%TL). Total length is expressed in millimeters. N: number of specimens; $x$ : mean; SD: standard deviation.

\begin{tabular}{|c|c|c|c|c|}
\hline & $\mathbf{N}$ & & $x$ & SD \\
\hline Total length (mm) & 10 & $165.0-952.0$ & 631.1 & 257.3 \\
\hline Precaudal length & 10 & $76.4-80.9$ & 78.9 & 1.3 \\
\hline Pre-second dorsal length & 10 & $56.2-62.3$ & 60.0 & 1.9 \\
\hline Pre-first dorsal length & 10 & $32.6-35.6$ & 33.8 & 1.0 \\
\hline Pre-vent length & 10 & $50.1-53.6$ & 51.7 & 1.1 \\
\hline Prepelvic length & 10 & $47.2-50.6$ & 49.0 & 1.1 \\
\hline Prepectoral length & 10 & $19.8-23.3$ & 21.3 & 1.2 \\
\hline Head length & 10 & $20.0-24.2$ & 21.9 & 1.2 \\
\hline Prebranchial length & 10 & $16.2-20.8$ & 18.3 & 1.2 \\
\hline Prespiracular length & 10 & $9.5-13.0$ & 11.5 & 1.0 \\
\hline Preorbital length & 10 & $6.3-8.2$ & 7.5 & 0.6 \\
\hline Prenarial length & 10 & $4.2-5.5$ & 5.0 & 0.4 \\
\hline Preoral length & 10 & $8.0-10.7$ & 9.5 & 0.8 \\
\hline Inner nostril-labial furrow space & 10 & $3.9-5.6$ & 4.4 & 0.6 \\
\hline Mouth width & 10 & $6.9-8.2$ & 7.4 & 0.4 \\
\hline Labial furrow length & 10 & $1.7-3.2$ & 2.3 & 0.4 \\
\hline Internarial space & 10 & $3.1-4.1$ & 3.7 & 0.3 \\
\hline Interorbital space & 10 & $6.9-9.6$ & 7.7 & 0.8 \\
\hline Eye length & 10 & $2.7-5.9$ & 3.8 & 1.1 \\
\hline Eye height & 10 & $1.4-2.7$ & 1.8 & 0.3 \\
\hline Spiracle length & 10 & $1.0-1.9$ & 1.3 & 0.3 \\
\hline First gill-slit height & 10 & $1.3-2.0$ & 1.7 & 0.2 \\
\hline Fifth gill-slit height & 10 & $2.0-2.6$ & 2.1 & 0.2 \\
\hline Interdorsal space & 10 & $16.2-22.1$ & 19.8 & 1.8 \\
\hline Dorsal-caudal space & 10 & $9.9-11.5$ & 10.9 & 0.5 \\
\hline Pectoral-pelvic space & 10 & $20.7-25.1$ & 23.3 & 1.6 \\
\hline Pelvic-caudal space & 10 & $20.4-22.6$ & 21.5 & 0.9 \\
\hline First dorsal-fin length & 10 & $10.6-12.4$ & 11.9 & 0.6 \\
\hline First dorsal-fin anterior margin & 10 & $8.8-10.9$ & 9.6 & 0.8 \\
\hline First dorsal-fin base length & 10 & $6.4-7.6$ & 7.1 & 0.4 \\
\hline First dorsal-fin height & 10 & $5.9-6.7$ & 6.3 & 0.3 \\
\hline First dorsal-fin inner margin & 10 & $4.4-5.3$ & 4.9 & 0.3 \\
\hline First dorsal-fin posterior margin & 10 & $5.0-7.2$ & 6.6 & 0.7 \\
\hline First dorsal-fin spine length & 8 & $1.0-2.0$ & 1.6 & 0.3 \\
\hline First dorsal-fin spine base width & 10 & $0.3-0.6$ & 0.4 & 0.1 \\
\hline Second dorsal-fin length & 10 & $9.6-13.7$ & 12.0 & 1.1 \\
\hline Second dorsal-fin anterior margin & 10 & $7.0-10.5$ & 8.7 & 0.9 \\
\hline Second dorsal-fin base length & 10 & $5.9-8.7$ & 7.5 & 0.8 \\
\hline Second dorsal-fin height & 10 & $3.6-5.0$ & 4.4 & 0.5 \\
\hline Second dorsal-fin inner margin & 10 & $3.7-4.8$ & 4.4 & 0.4 \\
\hline Second dorsal-fin posterior margin & 10 & $4.4-5.8$ & 5.0 & 0.4 \\
\hline Second dorsal-fin spine length & 9 & $1.9-3.5$ & 2.7 & 0.4 \\
\hline Second dorsal-fin spine base width & 10 & $0.4-1.0$ & 0.6 & 0.2 \\
\hline Pectoral-fin anterior margin length & 10 & $10.7-15.0$ & 13.8 & 1.4 \\
\hline Pectoral-fin inner margin length & 10 & $7.3-8.7$ & 7.8 & 0.4 \\
\hline Pectoral-fin base length & 10 & $3.5-4.9$ & 4.4 & 0.4 \\
\hline Pectoral-fin posterior margin length & 10 & $7.0-9.9$ & 9.1 & 0.8 \\
\hline Pelvic length & 10 & $9.3-12.5$ & 10.6 & 1.0 \\
\hline Pelvic-fin inner margin length & 10 & $3.5-5.6$ & 4.7 & 0.7 \\
\hline Dorsal caudal margin length & 10 & 19.3-21.9 & 21.0 & 0.8 \\
\hline Preventral caudal margin length & 10 & 9.9-20.8 & 11.9 & 3.2 \\
\hline Caudal fork width & 10 & $6.3-11.2$ & 7.3 & 1.4 \\
\hline Head width at nostrils & 10 & $5.6-8.0$ & 6.6 & 0.8 \\
\hline Head width at mouth & 10 & $9.1-11.3$ & 9.9 & 0.6 \\
\hline Head width & 10 & $9.5-12.6$ & 11.2 & 1.1 \\
\hline Trunk width & 10 & $6.6-13.6$ & 9.8 & 1.9 \\
\hline Abdomen width & 10 & $5.4-9.7$ & 7.9 & 1.6 \\
\hline Head height & 10 & $7.8-10.6$ & 9.1 & 0.9 \\
\hline Trunk height & 10 & 8.5-13.9 & 10.3 & 1.7 \\
\hline Abdomen height & 10 & $7.0-12.4$ & 9.7 & 1.9 \\
\hline Clasper outer length & 4 & $2.0-6.0$ & 5.0 & 2.0 \\
\hline
\end{tabular}


Japan; ZUMT 46674, neonate male, 270 mm TL, Uozu, Toyama Prefecture, Japan; ZUMT 46675, neonate male, $245 \mathrm{~mm}$ TL, Uozu, Toyama Prefecture, Japan; ZUMT 51256, neonate female, $165 \mathrm{~mm}$ TL, East China Sea.

Diagnosis. A large-sized Squalus species (650-952 mm maximum TL in adults) that can be differentiated from all its congeners (except $S$. acanthias) and including the Japanese species by: presence of rows of white spots dorsally on each side of the body (vs. absence of white spots) (Fig. 6F); anterior nasal flap unilobed (vs. bi-lobed); first dorsal fin origin located conspicuously behind the vertical line traced at pectoral-fin free rear tips (vs. first dorsal fin origin located before the vertical line); dermal denticles arrow-shaped (Fig. 7K, L) (vs. denticles rhomboid or lanceolate). It is further distinguished from the Japanese species by: shorter distance between pelvic and caudal fins $20.7 \%-22.6 \%$ TL, except with $S$. acutirostris (vs. $25.5 \%-27.3 \%$ TL for $S$. shiraii vs. $23.7 \%-27.0 \%$ TL for S. mitsukurii vs. $26.4 \%-28.0 \%$ TL for S. japonicus vs. $24.6 \%-29.4 \%$ TL for $S$. brevirostris); small first dorsal fin (except for S. japonicus), its length $11.7 \%-12.4 \%$ TL (vs. $12.6 \%-13.4 \%$ TL for $S$. shiraii vs. $12.7 \%-14.9 \%$ TL for $S$. mitsukurii vs. $12.3 \%-13.6 \%$ TL for S. japonicus vs. $13.0 \%-14.7 \%$ TL for $S$. brevirostris vs. $14.1 \%-15.0 \%$ TL for S. acutirostris); lower dorsal fins with first dorsal fin height $5.9 \%-6.7 \%$ TL, except with $S$. japonicus and $S$. acutirostris (vs. $7.9 \%-8.2 \%$ TL for $S$. shiraii vs. $6.9 \%-9.8 \%$ TL for $S$. mitsukurii vs. $7.3 \%-8.2 \%$ TL for S. brevirostris) and second dorsal fin height 3.6\%-4.9\% TL (except for S. mitsukurii and S. acutirostris) (vs. $5.6 \%-5.9 \%$ TL for $S$. shiraii vs. 5.4\%-6.5\% TL for $S$. japonicus vs. 5.7\%-6.9\% TL for $S$. brevirostris); conspicuously smaller dorsal-fin spines with first dorsal-fin spine length $1.0 \%-2.0 \%$ TL (vs. 3.6\%-4.3\% TL for $S$. shiraii vs. $3.1 \%-5.4 \%$ TL for $S$. mitsukurii vs. $3.0 \%-3.8 \%$ TL for $S$. japonicus vs. $3.1 \%-4.2 \%$ TL for $S$. brevirostris vs. $2.7 \%-2.8 \%$ TL for $S$. acutirostris) and second dorsal-fin spine $1.9 \%-3.2 \%$ TL, except with $S$. acutirostris (4.3\%-5.1\% TL for $S$. shiraii vs. $3.4 \%-5.3 \%$ TL for $S$. mitsukurii vs. $4.7 \%-5.6 \%$ TL for $S$. japonicus vs. $4.6 \%-$ $6.6 \%$ TL for $S$. brevirostris). It also has smaller interdorsal space $19.2 \%-22.1 \%$ TL than from $S$. shiraii sp. nov. (23.8\%-25.7\% TL), S. japonicus (22.5\%-24.8\% TL) and S. brevirostris $(23.3 \%-27.2 \% \mathrm{TL})$ and $S$. acutirostris (24.6\%-28.3\% TL) and more elongate pre-first dorsal length $32.6 \%-34.8 \%$ TL than $S$. shiraii sp. nov. (29.9\%$31.2 \%$ TL), S. mitsukurii (28.3\%-32.4\% TL), S. brevirostris $(28.9 \%-31.7 \%$ TL, and S. acutirostris $(31.8 \%-32.3 \%$ TL). It is further separated from its regional congeners by smaller number of precaudal (71-73) and diplospondylous vertebrae (58-62) (vs. larger number of vertebrae for S. shiraii sp. nov. 91-94 and 72-77 vs. 86-90 and 70-72 for $S$. mitsukurii vs. 80-88 and 65-75 for S. japonicus, vs. 78-89 and 66-72 for S. brevirostris).

Geographical distribution. This species is found in temperate and adjacent Arctic waters of the North Pacif- ic Ocean from Washington state and California, USA to Canada (east side) and South Korea to Russia (west side) (Veríssimo et al. 2010; Lee et al. 2016; Dyldin and Orlov 2018). In Japan, reports are from Hokaiddo, Notori Misaki oki and Uozu as well as Tokyo from where specimens were obtained in the local fish market. It occurs more commonly in temperate waters of Northern Japan (Fig. 8E).

Remarks. Molecular genetic data has supported the taxonomic separation between the morphologically similar species S. acanthias Linnaeus, 1758, that bears circumglobal distribution and S. suckleyi (e.g. Ward et al. 2007; Veríssimo et al. 2010; Ebert et al. 2010; Naylor et al. 2012; Lee et al. 2016). The latter species is a regional endemic to the North Pacific Ocean with distribution ranging from Korea to Japan (western side) and from California to Canada (east side). Morphological separation between these two species is still unreliable as no efficient diagnostic features were provided as yet. Lindberg and Legeza (1956) suggested that the ratio pelvic-fin midpoint to first dorsal-fin insertion (PDI) and pelvic-fin midpoint to second dorsal-fin insertion (PDO) comprise diagnostic characters as well as the first dorsal-fin midpoint to pectoral-fin insertion (DPI) and first dorsal-fin midpoint to pelvic-fin origin (Ebert et al. 2010); however, these parameters are subject to bias due to the midpoint definition that relies on subjectivity. Squamation, colouration and vertebral counts, however, separate $S$. acanthias and $S$. suckleyi (Table 6). The latter species exhibits dermal denticles with a median ridge forming an acute angle or right angle when perpendicular to the body axis (vs. in parallel for $S$. acanthias). It is easily distinguished from $S$. acanthias by having body conspicuously dark grey dorsally with very few white spots distributed dorsally in a single row on each side of the body (vs. light grey body with many white spots in two rows). Specimens of S. suckleyi from Japan are also distinct from $S$. acanthias occurring elsewhere by having smaller number of vertebrae (monospondylous, diplospondylous, precaudal and total vertebrae; see Springer \& Garrick, 1964 for S. acanthias), corroborating Ebert et al. (2010).

Additional characteristics of $S$. suckleyi include: dermal denticles arrow-shaped and unicuspid, small and not imbricated with a single ridge (lateral ridges absent); ridge very prominent, narrower distally than proximally with furrow anterior and profound; ridge very tall and convex, forming 45 degrees angle with horizontal axis of the body; crown base strongly broad and diamond-like with four prominent pedicels; dermal denticles conspicuously expanded laterally at the crown (Fig. 7K, L). A single adult male shows few dermal denticles with weak lateral cusps, although lateral ridges are still absent. Colouration of Japanese specimens consists of body dark grey dorsally with very few and inconspicuous white spots (1-6 pairs), rounded, distributed symmetrically in a single row on each side and laterally at the body; white spots commonly absent in some large specimens, although clearly evident in young juveniles; white to greyish-white ven- 
trally. Both dorsal fins grey, brownish in the apex, slightly darker from the apex tip to the midline of the posterior margin, white distally at posterior margin and free rear tips; fin base slightly white; dorsal spines dark brown, white at the tip. Pectoral fins dark grey dorsally and ventrally with white posterior margin, although not uniform. Pelvic fins also dark grey with posterior and inner margins slightly white. Caudal fin grey, darker at the tip of the dorsal lobe and in the lower caudal lobe, white over vertebral column; proximal end of dorsal caudal margin white; postventral caudal margins narrowly white; preventral caudal margin slightly white; black caudal stripe conspicuous above vertebral column.

Variations of diet and reproduction, as well as migration pattern, spatial distribution and size composition, were noticed in populations of $S$. suckleyi between the East and West sides of the North Pacific Ocean (Orlov et al. 2012a,b; Yano et al. 2017), suggesting that this species may comprise more than one fishing stock rather than a single one as supported in Ebert et al. (2010). In contrast to $S$. acanthias, this species has low migration movements and usually inhabits the pelagic zones up to 200 $m$ depth with two possibly distinct populations observed throughout the North Pacific Ocean (Yano et al. 2017). Emperor Seamout Chain works as a biogeographic barrier between the East and West sides whose belt-shaped pelagic zone exhibits low probability of presence of $S$. suckleyi, according to Yano et al. (2017).

A single nominal species of spotted spiny dogfish, Squalus wakiyae Tanaka, 1917, was described from the North-western Pacific Ocean and it has been placed under synonymy with $S$. acanthias and S. suckleyi in Fowler (1941), White et al. (2007c), Ebert et al. (2010) and the present study, as no apparent differences in morphology and genetic data are noticed between the west and east populations of S. suckleyi. Morphometric variations, however, are noticed when comparing the neotype (taken from Ebert et al. 2010) to specimens from off Japan and Russia: greater prepelvic length than in specimens from the NWPO (51.5\% TL for neotype vs. $47.2 \%-50.6 \%$ TL); greater pre-vent length ( $54.9 \%$ TL vs. $50.1 \%-53.6 \%$ TL); narrower mouth $(6.2 \%$ TL vs. $6.9 \%-8.2 \%$ TL); lower head height $(6.5 \%$ TL vs. $7.8 \%-10.6 \%$ TL); pectoral fin broader at the base $(6.4 \%$ TL vs. $3.5 \%-4.9 \%$ TL); lower second dorsal fin in S. suckleyi $(3.0 \%$ TL vs. 3.6\%-5.0\% TL). A larger number of comparative representatives are required to re-evaluate these variations between populations of $S$. suckleyi and should include additional characteristics, such as dentition and vertebral counts.

Comparative material. S. acanthias: BMNH 1879.10.9.64, adult female, $620 \mathrm{~mm}$ TL, off east coast, The United States of America; BMNH 1929.10.20.1, adult female, 875 mm TL, United Kingdom; BMNH 1931.4.27.2, adult female, $800 \mathrm{~mm}$ TL, United Kingdom; BMNH 1936.8.26.17, adult male, $635 \mathrm{~mm}$ TL, near Strait of Magellan, Argentina; BMNH 1950.7.26.2, juvenile female, 570 mm TL, Republic of Ireland; BMNH 1976.7.30.20, juvenile female, $523 \mathrm{~mm}$ TL, France, Mediterranean Sea; BMNH 1999.5.4.4, juvenile male, $550 \mathrm{~mm}$ TL, Falkland Islands; NMW 50119, adult female, 910 mm TL, Dalmatien, Croatia; NMW 59659, juvenile male, 345 mm TL, The North Sea; CSIRO H 2921-01, adult female, 605 mm TL, upper Pitt Water, near Shark Point, Tasmania, Australia; NMW 84781, juvenile female, $525 \mathrm{~mm}$ TL, Trieste, Italy; NSMT-P 41928, adult female, 715 mm TL, Atlantic Ocean; HUMZ 151302, juvenile male, 520 mm TL, off Namibia; HUMZ 30295, adult male, $660 \mathrm{~mm}$ TL, off Patagonia, Argentina; HUMZ 65447, adult male, $755 \mathrm{~mm}$ TL, New Zealand; SAIAB 21877, juvenile male, $590 \mathrm{~mm}$ TL, Cape Columbine, South Africa; SAIAB 25317, adult male, $640 \mathrm{~mm}$ TL, west coast of South Africa.

\section{Discussion}

Despite the fact that the taxonomy of Japanese dogfish sharks has been previously well discussed (e.g. Chen et al. 1979; Shirai 1989) and the species accounts constantly updated (e.g. Shinohara et al. 2014), the identification and validation of certain species were still unclear until the present. This study provides a revisited evaluation of Squalus species occurring in Japan and adjacent areas, following recent trends in the Systematics of the group with description of a novel species.

Characteristics of the external morphology, morphometric and meristic data support the separation and identification of the Japanese species which is congruent with previously similar work on the genus (e.g. Last et al. 2007f; Viana et al. 2016; Viana and Carvalho 2018b). Squalus shiraii sp. nov. bears many characteristics that distinctly separates it from its regional congeners as seen in the differential diagnosis provided. These characteristics are mostly related to external measurements of the body (e.g. height of dorsal fins), body colouration (e.g. dorsal and ventral caudal tips broadly white; body markedly dark grey in colour) and vertebral counts (e.g. precaudal and total vertebrae). Our results reveal that Squalus shiraii is morphologically similar to species of the "highfin megalops group" (S. albifrons, S. altipinnis, S. formosus and $S$. notocaudatus) in having dorsal fins conspicuously tall and upright and dorsal-fin spines robust and elongate. $S$. shiraii has postventral caudal margins not uniformly white with conspicuous black caudal bar, dermal denticles unicuspidate and lanceolate and snout markedly elongate and pointed at tip which are distinct from these species (vs. postventral caudal margins uniformly white without black caudal bar; denticles tricuspidate and rhomdoid, see Fig. 7C, D; snout small and rounded at tip). It lacks a white upper ocular margin and white bar at anterior margin of first dorsal fin that is diagnostic for $S$. albifrons and $S$. formosus, respectively. The latter species has been previously misidentified with $S$. blainvillei in Japan but White and Iglésias (2011) noticed that the characteristics in Chen et al. (1979) overlap those of the Taiwanese species, especially regarding having body greyish-brown 

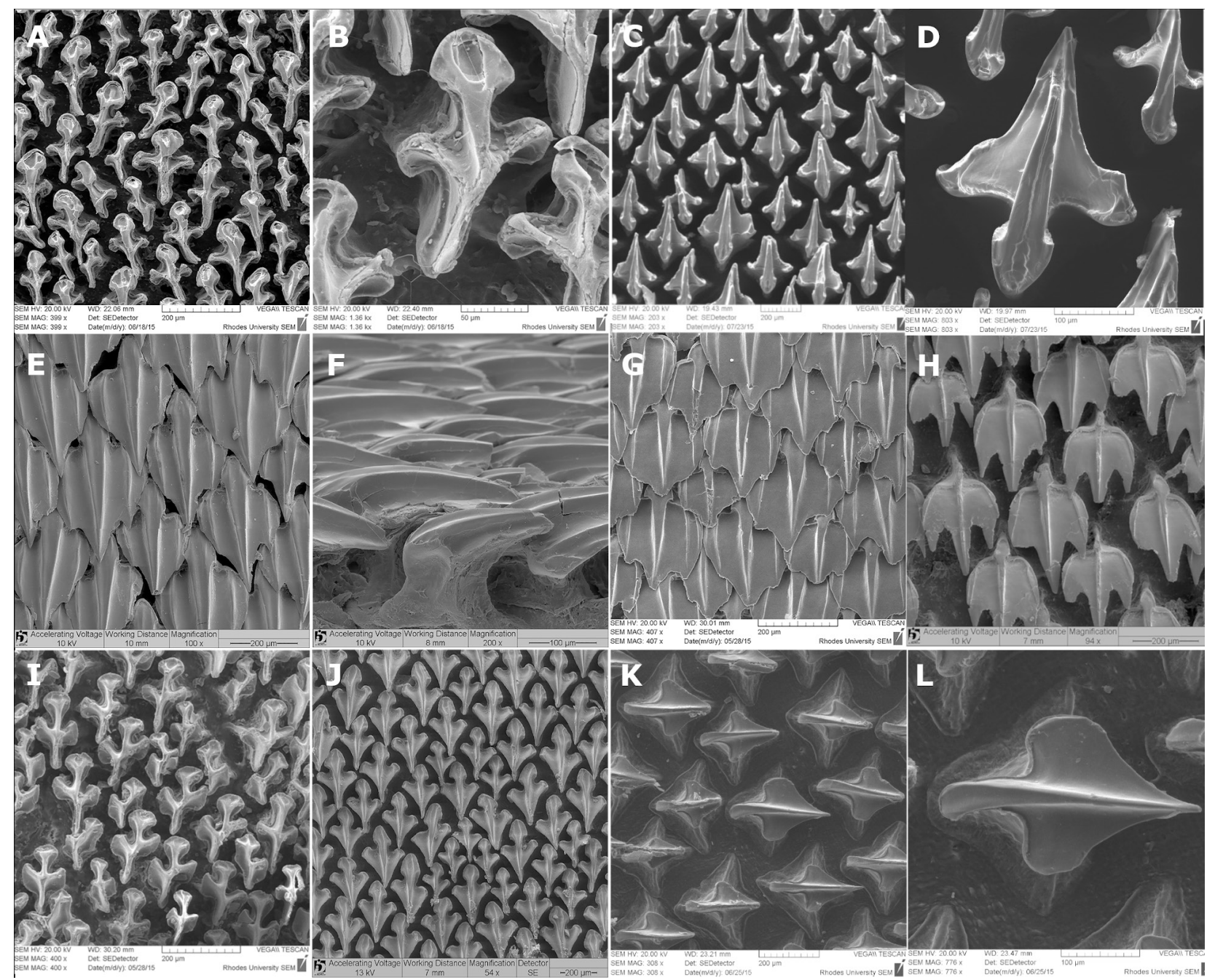

Figure 7. Scanning electron microscopy of the dermal denticles. Squalus shiraii sp. nov. (A, B): HUMZ 149389 (holotype), adult male, 590 mm TL. S. formosus (C, D): CSIRO H-6816-01 (holotype), adult male, 720 mm TL. S. mitsukurii (E, F): HUMZ 102987, adult male, 970 mm TL. S. japonicus (G, H): NSMT-P 44380 (neotype), adult male, 645 mm TL (G); NSMT-P 91127, juvenile female, $410 \mathrm{~mm}$ TL (H). S. brevirostris (I, J): NSMT-P 47378, adult female, $600 \mathrm{~mm}$ TL (I); KAUM-I 187, adult female, $578 \mathrm{~mm}$ TL (J). S. suckleyi (K, L): NSMT P-92640, adult female, 970 mm TL. Scale bars: 50 $\mu \mathrm{m}(\mathbf{B}) ; 100 \mu \mathrm{m}(\mathbf{D}, \mathbf{F}, \mathbf{L}) ; 200 \mu \mathrm{m}(\mathbf{A}, \mathbf{C}, \mathbf{E}, \mathbf{G}-\mathbf{K})$.

dorsally and pale ventrally, first dorsal fin with anterior margin conspicuously white and vertebral counts. With the exception to vertebrae, Squalus shiraii has a variety of characteristics that separates it from $S$. formosus besides those listed here, including larger snout with nostrils closer to mouth and snout tip, smaller and lower dorsal fins and smaller pelvic fins as noticed in the diagnosis and remarks above. The distribution of S. formosus in Japanese waters is still uncertain as no specimen data were found in the ichthyological collections visited and, thus, this species was not included in the present revision.

Squalus shiraii has also been confused with $S$. mitsukurii in the area in previous taxonomic accounts because they share body robust, elongate snout and dark caudal bar and/or black upper caudal blotch, evident in at least one stage of maturity. The current analysis shows that they are non-conspecific by having clear morphological separation, based on vertebral counts (precaudal and total vertebrae), body colouration and morphometrics. Squalus mitsukurii is supported here as a regional endemic species to the North-western Pacific Ocean, which is in disagreement with Bigelow and Schroeder (1948, 1957), Bass et al. (1976), Compagno (1984) and Compagno et al. (2005a) and which was previously known as cosmopolitan. This result is congruent with recent taxonomic investigations on this species of Viana et al. (2017b), Veríssimo et al. (2017), Pfleger et al. (2018) and Daly-Engel et al. (2018) who refuted the occurrence of $S$. mitsukurii in the Western Indian, Western and Eastern Atlantic Oceans. The present analysis also rejects the occurrence of $S$. mitsukurii in Hawaiian waters given the validity of $S$. hawaiiensis, as supported in Daly-Engel et al. (2018) and in the diagnosis and remarks above. The recognition of $S$. hawaiiensis and $S$. boretzi in the North Pacific Ocean, as well as $S$. shiraii sp. nov. that exhibit closely sympatric distribution with $S$. mitsukurii, represent the still ongoing taxonomic confusion behind this species even in the surrounding topotypic region. Single or combined taxonomic evidence (e.g. 


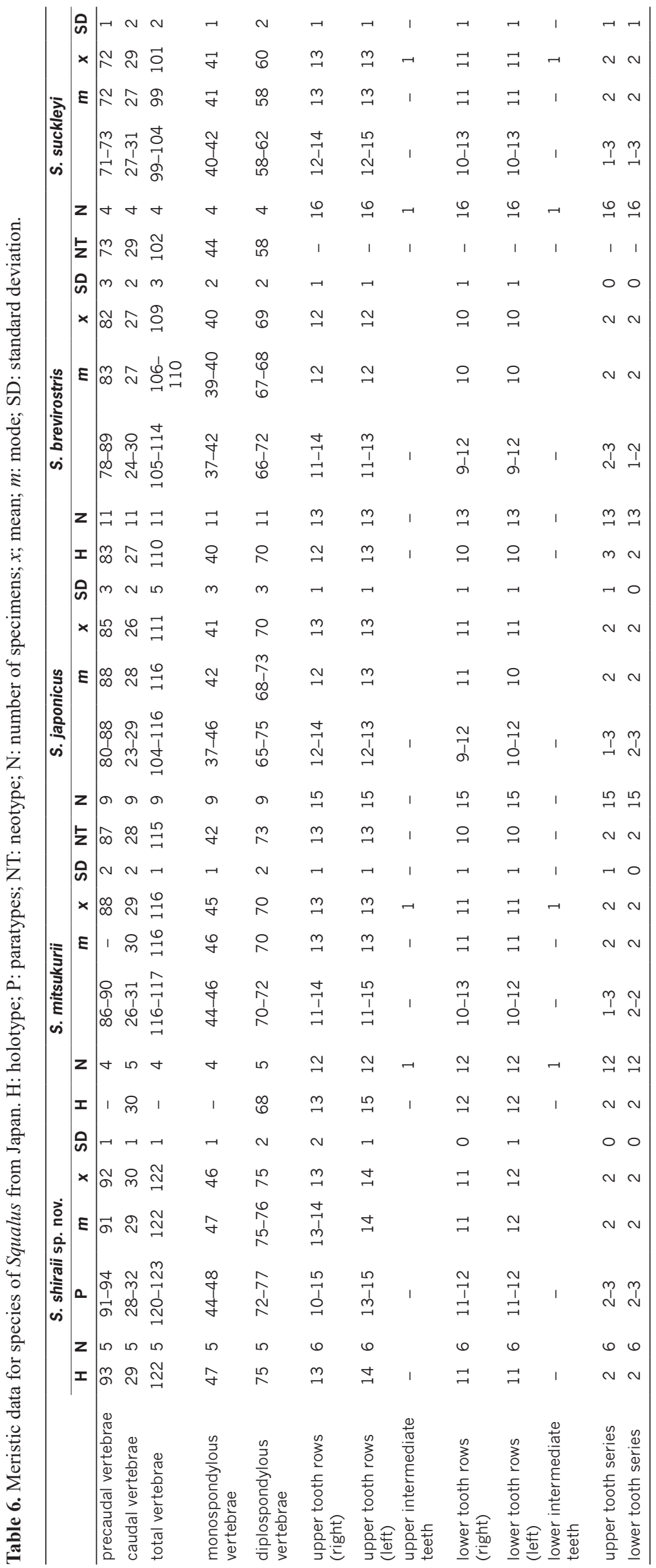


morphological and molecular data) have supported current and constant changes in the taxonomy of S. mitsukurii as seen in Viana et al. (2017b), Pfleger et al. (2018), Daly-Engel et al. (2018) and Dolganov (2019). Thus, reporting occurrences of this species elsewhere, including Nazca and Sala y Gomez seamounts, Chile, India, Mexico (east coast), Costa Rica, Madagascar, Colombia and Uruguay in Parin (1987), Dyer and Westneat (2010), Akhilesh et al. (2014), Del Moral-Flores et al. (2015, 2016), Ehemann et al. (2018), Espinoza et al. (2018), Fricke et al. (2018), Mejía-Falla and Navia (2019) and Nión and Meneses (2016) require taxonomic clarification. It is more likely that it has been confused either with a variety of the latest Squalus species that were described or resurrected in the past 10 years or may represent undescribed species with a possible degree of regionalism/endemism. Another nominal species, Squalus acutirostris, was also misidentified with $S$. mitsukurii, but it is regarded here as a valid species with occurrences in China, Japan, Taiwan and Vietnam. Squalus acutirostris seems to be rarely caught in Japanese waters once the availability of specimen data in the local ichthyological collections that were visited is extremely low. Due to limited availability of specimen data for this species, a tentative diagnosis was provided. A detailed re-description of this nominal species is urgently required, based on new material from the areas in which this species has been reported in order to improve its morphological characterisation and better comprehend the separation with its regional congeners. Investigations on other aspects of $S$. acutirostris (e.g. vertebral counts, dentition, dermal denticles) is crucial.

Squalus japonicus is endemic to the Indo-West Pacific Ocean from Japan and Taiwan. Its occurrence in the Eastern Pacific Ocean, off waters from Australia, New Zealand, New Caledonia and Vanuatu are not observed in the present study. These findings are in congruence with Compagno (1984, 2000), Compagno and Niem (1998), Compagno et al. (2005a), Last et al. (2007b), Yamashita et al. (2012), Motomura et al. (2015) and Viana and Carvalho (2018a). Squalus japonicus is very similar in morphology to $S$. nasutus and S. melanurus for sharing a body conspicuously thin from head to tail, head elongate, snout markedly elongate and obtuse, mouth narrow and pectoral fins tapered. These three species exhibit the greatest values of some head measurements (e.g. prebranchial length, preorbital length, preoral length, prenarial length) when compared to all other Squalus congeners, including S. shiraii.

Out of the six species examined in the present study, S. brevirostris appears to be the one with highest degree of taxonomic confusion. Difficulties in adequately identifying this species were widely reported (e.g. Garrick 1960; Chen et al. 1979; Compagno 1984; Last et al. 2007a; Straube et al. 2013) and were most likely due to intraspecific variations that were noticed here in the shape of denticles and pectoral fins, as well as caudal colouration. These variations, however, are not uniform and no apparent morphological pattern has been detected throughout the overall distribution of this species, even when maturity and sex are considered. Squalus brevirostris was thought to extend its occurrence to Australian waters in Chen et al. (1979), but it was refuted in Last et al. (2007) which is corroborated here. This species differs from $S$. megalops by having the first dorsal fin raked with posterior margin concave near the apex, body brownish-grey, postventral caudal margins broadly white, while $S$. megalops shows first dorsal fin not raked with posterior margin straight, body grey and postventral caudal margins narrowly white. It can be further separated from the Australian species and other closely-related species, including $S$. acutipinnis, $S$. crassispinus, $S$. albifrons, $S$. notocaudatus, $S$. raoulensis, $S$. hemipinnis and $S$. bucephalus by having pectoral-fin free rear tips markedly pointed (vs. pectoral-fin free rear tips rounded). Squalus brevirostris has small pectoral fins, never transcending the trunk height, while in $S$. raoulensis, the pectoral fins are conspicuously broad, always transcending the trunk height when adpressed on body. Dorsal fins are low and oblique in $S$. brevirostris while $S$. albifrons, $S$. crassispinus, $S$. formosus, S. notocaudatus, $S$. raoulensis, S. hemipinnis and $S$. bucephalus exhibit conspicuously high and upright dorsal fins. S. brevirostris lacks whitish eyebrow, a diagnostic characteristic of $S$. albifrons and it has very thin dorsal-fin spines (vs. heavy dorsal-fin spines in $S$. crassispinus). These findings support the fact that the distribution of $S$. brevirostris is restricted to the North and Central Western Pacific Oceans and is in congruence with Duffy and Last (2007a), Yamashita et al. (2012), Naylor et al. (2012), Straube et al. (2013) and Shinohara et al. (2014).

Species delimitation through molecular data is still unavailable for Squalus from Japan and surrounding countries as genetic information on the species through topotypic voucher specimens are still uncommon, except for $S$. brevirostris and S. suckleyi (e.g. Ward et al. 2007; Naylor et al. 2012; Straube et al. 2013; Veríssimo et al. 2017) and S. mitsukurii and S. japonicus (e.g. Pfleger et al. 2018; Daly-Engel et al. 2018) that have been barcoded in recent years. Thus, molecular analysis of mitochondrial genes for S. shiraii and S. acutirostris are lacking. Surprisingly, studies that have included molecular data of Japanese species did not suggest occurrence of possible undescribed or unidentified species in the region, indicating that insufficient data-collecting effort throughout the species distribution possibly do not cover the local diversity. The recognition of $S$. shiraii as a novel species and the present taxonomic revision represent the latest comprehensive evaluation of Japanese Squalus species to date and highlights that further efforts in elucidating the complexity behind the genus in other countries in Asia (e.g. South Korea, Philippines, Vietnam, China) are still required. Aspects of the biology, distribution, ecology and population size of Squalus species from Japan are still poorly acknowledged with only two species, S. mitsukurii and S. suckleyi, usually targeted and more rarely S. japonicus (e.g. Chen et al. 1981; Orlov et al. 2011, 2012; Cotton et al. 2011; Yano et al. 2017). Understanding the related threats and population trends are paramount as these species are historically under fishing pressures in Japanese waters. 


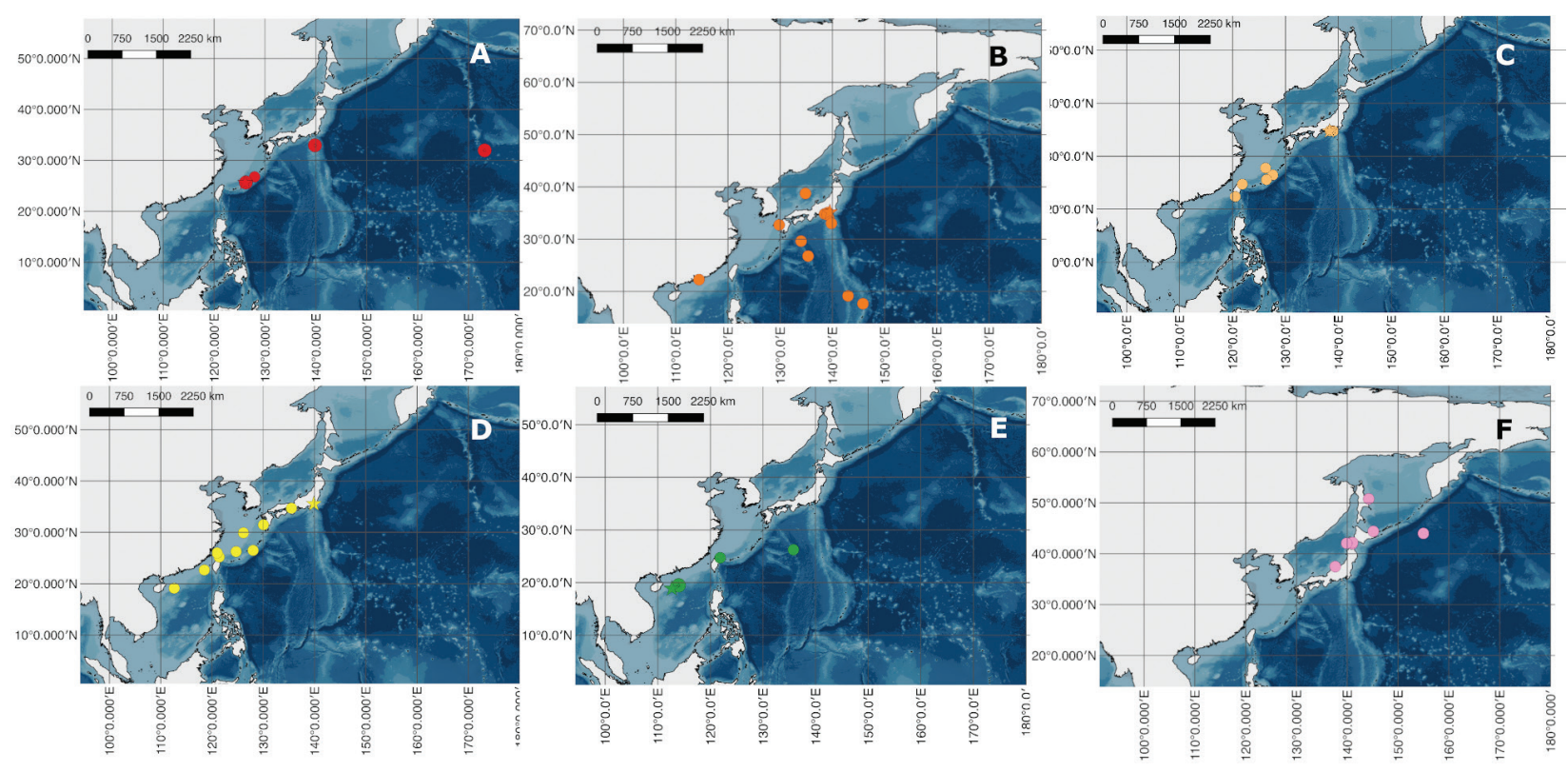

Figure 8. Map of the North-western Pacific Ocean, showing the geographical distribution of Squalus species. A: S. shiraii sp.nov. (red); B: S. mitsukurii (orange); C: S. japonicus (brown); D: S. brevirostris (yellow); E: S. acutirostris (green); F: S. suckleyi (pink). Star: holotype/ neotype; Dotted circle: paratypes; Circle: other material.

\section{Identification key to Squalus species from Japan}

1a Origin of first dorsal fin placed posterior to vertical line traced at pectoral-fin free rear tips; anterior margin of nostrils unilobed; body with row of white spots dorsally; dermal denticles arrow-shaped; pectoral-caudal distance $20.7 \%-22.6 \%$ TL; first dorsal-fin spine 1.0\%-2.0\% TL; first dorsal fin height 5.9\%-6.7\% TL ..... S. suckleyi

$1 \mathrm{~b}$ Origin of first dorsal fin placed anteriorly to vertical traced at pectoral-fin free rear tips; anterior margin of nostrils bi-lobed; body without white spots dorsally; dermal denticles rhomboid or lanceolate; pectoral-caudal distance $23.7 \%-28.0 \%$ TL; first dorsal-fin spine $2.7 \%-5.4 \% \mathrm{TL}$; first dorsal fin height $5.6 \%-9.8 \% \mathrm{TL}$

2a Small sized body 373-578 mm TL; snout conspicuously small with prenarial length 0.8-0.9 times inner nostril-labial furrow space; pectoral-fin free rear tips conspicuously pointed; postventral caudal margins uniformly white; caudal fin without black caudal bar and/or black upper caudal blotch; dermal denticles unicuspid and lanceolate; few number of monospondylous vertebrae (37-42) and total vertebrae (105-114), except with S. japonicus......

S. brevirostris

2b Medium to large sized body (512.0-1120 mm TL); snout conspicuously elongate with prenarial length 1.1-1.5 times inner nostrils-labial furrow space (except for S. acutirostris); pectoral-fin free rear tips conspicuously rounded; postventral caudal margins not uniformly white; caudal fin with black caudal bar and/or black upper caudal blotch; dermal denticles tricuspid and rhomboid (except for S. shiraii sp. nov.); high number of monospondylous vertebrae (44-48) and total vertebrae (116-123) ...3

3a Body markedly thin throughout (head width at mouth 9.7\%-10.7\% TL); snout conspicuously elongate (prenarial length 5.9\%-7.5\% TL); preoral length 10.7\%-12.8\% TL; mouth markedly narrow; pectoral fins conspicuously tapered (pecto. ral-fin posterior margin length $7.2 \%-9.2 \% \mathrm{TL}$ )

S. japonicus

3b Body markedly stout throughout (head width at mouth $10.7 \%-12.2 \% \mathrm{TL}$ ); snout moderately elongate (prenarial length 4.1\%-5.6\% TL); preoral length 7.6\%-10.4\% TL; mouth markedly broad; pectoral fins conspicuously wide (pectoral-fin posterior margin length $9.9 \%-12.5 \% \mathrm{TL}$ )

4a Body brown to light brown; snout markedly obtuse at tip; first dorsal fin conspicuously upright and tall; first dorsal fin with posterior margin markedly concave; pectoral fins conspicuously falcate; caudal fin tapered with dorsal and ventral caudal tips pointed and broadly white; dermal denticles unicuspidate and lanceolate; 91-94 precaudal and 120-123 total vertebrae.

S. shiraii sp. nov.

4b Body brownish-grey to dark grey or black; snout markedly rounded at tip; first dorsal fin oblique and low; first dorsal fin with posterior margin straight to slight concave; pectoral fins not falcate; caudal fin wide with dorsal and ventral caudal tips rounded and not broadly white; dermal denticles tricuspidate and rhomboid; 86-90 precaudal and 116-117 total vertebrae........5

$5 a$ Body robust and black to dark grey in colour; elongate snout; inner nostril-labial furrow space 3.9\%-5.3\% TL; tall dorsal fins (height of first dorsal fin 6.9\%-9.8\% TL, height of second dorsal fin 4.5\%-7.9\% TL); large second dorsal-fin spine, its length 3.3\%-5.3\% TL; caudal fins without dark caudal bar and upper caudal blotch in adults S. mitsukurii

5b Body thin and brownish-grey in colour; small snout; inner nostril-labial furrow space $6.9 \%-8.6 \%$ TL; low dorsal fins (height of first dorsal fin 5.6\%-6.0\% TL, height of second dorsal fin height 3.3\%-3.6\% TL; short second dorsal-fin spine, its length 1.9\%-2.8\% TL; caudal fins with dark caudal bar and upper caudal blotch in adults ..... S. acutirostris 


\section{Conclusions}

Six species of dogfish sharks occur in Japan according to the present study: S. mitsukurii, S. japonicus, S. brevirostris, S. acutirostris, S. suckleyi and S. shiraii sp. nov (Fig. 8 ). Occurrence of $S$. formosus is still doubtful as no specimen caught from the country was found in the ichthyological collections visited. One or more of these species are sympatric in the area, as well as in waters surrounding China, South Korea, Taiwan, Philippines, Vietnam and Russia as seen in the above results. The previous hypothesis on the occurrence of $S$. megalops and $S$. blainvillei in Japan is thus rejected herein. Morphological, meristics and morphometric data support the separation between the species included in the analysis and it is congruent with the latest studies on the Squalus taxonomy in other regions of the world. The present analysis furthermore points out that two species, S. acutirostris and S. shiraii, were not previously recognised in Japan in neither morphological and/or combined molecular taxonomic accounts which warrants the need of improvements in the availability of specimen data.

\section{Acknowledgements}

The author thanks M. Nakae (NSMT), T. Kawai and students (HUMZ), K. Sakamoto (ZUMT), H. Motomura (KAUM), H.-C. Ho (NMMB), A. Graham, W.T. White and P.R. Last (CSIRO), M. Stiassny and R. Schelly (AMNH), M. McGrouther, A. Hay and S. Reader (AMS), L. Rocha and D. Catania (CAS), L. Page and G. Burgess (FLMNH), L. Parenti and J. Williams (USNM), R. Thiel and I. Eidus (ZMH), O. Gon and R. Bills (SAIAB) for curatorial and technical assistance. Special thanks to M.T.P. Ragazzo (USP) for academic support and comments on this manuscript. K. Sakamoto (ZUMT), G. Shinohara, M. Nakae, F. Tashiro and K. Kuriiwa (NSMT), J. Pogonoski (CSIRO), S. Raredon (USNM) and J. Fong (CAS) are thanked for providing radiographs. F.F. Pettean (UFRN) is thanked for gathering data from types of S. acutirostris. E. Matos and P. Lenktaitis (USP), S. Pinchuck and M. Randall (RU) are thanked for assisting with SEM and M. Lisher (UJ) is thanked for English editing of this manuscript and taxonomic inputs. SV was supported by the Fundação de Amparo a Pesquisa do Estado de São Paulo (FAPESP, 2011/18861-7, 2013/11621-6, 2014/26503-1), Conselho Nacional de Desenvolvimento Científico e Tecnológico (CNPq, 158773/2011-0) and Geddes Collection Visiting Fellowship, Australian Museum (2013). The second author was supported by a grant from CNPq (305271/2015-6).

\section{References}

Akhilesh KV, Bineesh KK, Gopalakrishnan A, Jena JK, Basheer VS, Pillai NGK (2014) Checklist of Chondrichthyans in Indian waters. Journal of the Marine Biological Association of India 56(1): 109120. https://doi.org/10.6024/jmbai.2014.56.1.01750s-17
Baranes A (2003) Sharks from the Amirantes Islands, Seychelles, with a description of two new species of squaloids from the deep sea. Israel Journal of Zoology 49: 33-65. https://doi.org/10.1560/N4KUAV5L-0VFE-83DL

Bass AJ, D’Aubrey JD, Kistnasamy N (1976) Sharks of the east coast of southern Africa. VI The families Oxynotidae, Squalidae, Dalatiidae and Echinorhinidae. Investigational Report, Oceanographic Research Institute 45: 1-103.

Bigelow HB, Schroeder WC (1948) Sharks. In: J Tee-Van, et al. (Eds) Fishes of the Western North Atlantic. Part 1. Memoirs of the Sears Foundation for Marine Research, Yale University, New Haven, $1-576$.

Bigelow HB, Schroeder WC (1957) A study of the sharks of the suborder Squaloidea. Bulletin of the Museum of Comparative Zoology 117(1): 1-150.

Bineesh KK, Gopalakrishnan A, Akhilesh KV, Sajeela KA, Abdussamad EM, Pillai NGK, Basheer VS, Jena JK, Ward RD (2017) DNA barcoding reveals species composition of sharks and rays in the Indian commercial fishery. Mitochondrial DNA Part A 28(4): 458-472. https://oi.org/10.3109/19401736.2015.1137900

Cappetta H (1987) Chondrichthyes II: Mesozoic and Cenozoic Elasmobranchii. In: Schultze HP (Ed.) Handbook of Paleoichthyology, vol. 3B, Verlag Dr. Friedrich Pfeil, München, 1-193.

Chen CT, Taniuchi T, Nose Y (1979) Blainville's dogfish, Squalus blainvillei, from Japan, with notes on S. mitsukurii and S. japonicus. Japanese Journal of Ichthyology 26(1): 26-42.

Chen CT, Taniuchi Y, Nose Y (1981) Some aspects of reproduction in the pointed-snout dogfish Squalus japonicus taken off Nagasaki and Choshi. Bulletin of the Japanese Society of Scientific Fisheries 47(9): 1157-1164. https://doi.org/10.2331/suisan.47.1157

Compagno LJV (1984) FAO Species Catalogue No.125: Sharks of the world, an annotated and illustrated catalogue of shark species known to date. Part 1. Hexanchiformes to Lamniformes, vol. 4. FAO, Rome

Compagno LJV (1988) Sharks of the Order Carcharhiniformes. Princeton University Press, Princeton.

Compagno LJV (2000) Elasmobranchii. In: Randall JE, Lim KKP (Eds) A checklist of the fishes of the South China Sea. The Raffles Bulletin of Zoology 8. National University of Singapore, Singapore, 577-584.

Compagno LJV (2002) Sharks. In: Carpenter KE (Ed.) The living marine resources of the Western Central Atlantic. Vol. 1. Introduction, molluscs, crustaceans, hagfishes, sharks, batoid fishes and chimaeras. FAO Species identification guide for fishery purposes and American Society of Ichthyologists Special Publication 5. FAO, Rome, 357-505.

Compagno LJV, Dando M, Fowler S (2005a) Sharks of the World. Princeton University Press, New Jersey.

Compagno LJV, Last PR, Stevens JD, Alava MNR (2005b) Checklist of Philippine Chondrichthyes. CSIRO Marine Laboratories Report 243, Hobart.

Compagno LJV, Niem VH (1998) Several family accounts. In: Carpenter KE, Niem VH (Eds) FAO species identification guide for fishery purposes. The living marine resources of the western central pacific. Cephalopods, crustaceans, holothurans and sharks 2. FAO, Senckenbergiana Biologica, Rome, 687-1396.

Cotton CF, Grubbs RD, Daly-Engel TS, Lynch PD, Musick JA (2011) Age, growth and reproduction of Squalus cf. mitsukurii from Hawaiian waters. Marine and Freshwater Research 62: 811-822. https:// doi.org/10.1071/MF10307 
Daly-Engel TS, Koch A, Anderson JM, Cotton CF, Grubbs RD (2018) Description of a new deep-water dogfish shark from Hawaii, with comments on the Squalus mitsukurii species complex in the West Pacific. ZooKeys 798: 135-157. https://doi.org/10.3897/zookeys.798.28375

da Silva C, Booth AJ, Dudley SFJ, Kerwath SE, Lamberth SJ, Leslie RW, McCord ME, Sauer WHH, Zweig T (2015) The current status and management of South Africa's chondrichthyan fisheries. African Journal of Marine Science 37(2): 233-248. https://doi.org/10.29 89/1814232X.2015.1044471

Del Moral-Flores LF, Morrone JJ, Alcocer Durand J, Espinosa-Pérez H, Pérez-Ponce De León G (2015) Lista patrón de los tiburones, rayas y quimeras (Chondrichthyes, Elasmobranchii, Holocephali) de México. Arxius de Miscellania Zoològica 13: 47-163. https://doi. org/10.32800/amz.2015.13.0047

Del Moral-Flores LF, Morrone JJ, Alcocer Durand J, Pérez-Ponce De León G (2016) Diversidad y afinidades biogeográficas de los tiburones, rayas y quimeras (Chondrichthyes: Elasmobranchii, Holocephali) de México. Revista de Biología Tropical 64(4): 1469-1486. https://doi.org/10.15517/rbt.v64i4.22774

Deynat PP, Séret B (1996) Le revêtement cutané des raies (Chondrichthyes, Elasmobranchii, Batoidea). I: Morphologie et arrangement des denticules cutanés. Annales de Sciences Naturelles, Zoologie et Biologie Animale 17(2): 65-83.

Dolganov VN (2019) Squalus boretzi sp. n. (Squalidae), a new squalid shark species from the Emperor Seamount Chain, Pacific Ocean (in Russian). Biologiya Morya (Vladivostok) 45(4): 279-285. https:// doi.org/10.1134/S0032945219030044

Domeier M, Shephard GE, Jakob J, Gaina C, Doubrovine PV, Torsvik TH (2017) Intraoceanic subduction spanned the Pacific in the Late Cretaceous-Paleocene. Science Advances 3: eaao2303. https://doi. org/10.1126/sciadv.aao2303

Duffy CAJ, Last PR (2007a) Part 4 - Squalus raoulensis sp. nov., a new spurdog of the megalops-cubensis group from the Kermadec Ridge. In: Last PR, White WT, Pogonoski JJ (Eds) Descriptions of New Dogfishes of the Genus Squalus (Squaloidea: Squalidae). CSIRO Marine and Atmospheric Research Paper 14, Hobart, 31-38.

Duffy CAJ, Last PR (2007b) Part 9 - Redescription of the Northern Spiny Dogfish Squalus griffini Phillipps, 1931 from New Zealand. In: Last PR, White WT, Pogonoski JJ (Eds) Descriptions of New Dogfishes of the Genus Squalus (Squaloidea: Squalidae). CSIRO Marine and Atmospheric Research Paper, 14, Hobart, 91-100.

Dulvy NK, Simpfendorfer CA, Davidson LNK, Fordham SV, Brautigam A, Sant G, Welch DJ (2017) Challenges and priorities in shark and ray conservation. Current Biology Minireview 27: 565-572. https://doi.org/10.1016/j.cub.2017.04.038

Dyer BS, Westneat MW (2010) Taxonomy and biogeography of the coastal fishes of Juan Fernández Archipelago and Desventuradas Islands, Chile. Revista de Biología Marina y Oceanografía 45(S1): 589-617. https://doi.org/10.4067/S0718-19572010000400007

Dyldin YV (2015) Annotated checklist of the sharks, batoids and chimaeras (Chondrichthyes: Elasmobranchii, Holocephali) from waters of Russia and adjacent areas. Publications of the Seto Marine Biological Laboratory 43: 40-91. https://doi.org/10.5134/197957

Dyldin YV, Orlov AM (2018) An annotated list of cartilaginous fishes (Chondrichthyes: Elasmobranchii, Holocephali) of the coastal waters of Sakhalin Island and the adjacent southern part of the Sea of Okhotsk. Journal of Ichthyology 58(2): 158-180. https://doi. org/10.1134/S0032945218020042
Ebert DA, HO H-C, White WT, Carvalho MR (2013) Introduction to the systematics and biodiversity of sharks, rays, and chimaeras (Chondrichthyes) of Taiwan. Zootaxa 3752: 5-19. https://doi. org/10.11646/zootaxa.3752.1.3

Ebert DA, White WT, Goldman KJ, Compagno LJV, Daly-Engel TS, Ward RD (2010) Resurrection and redescription of Squalus suckleyi (Girard, 1854) from the North Pacific, with comments on the Squalus acanthias subgroup (Squaliformes: Squalidae). Zootaxa 2612: 22-40. https://doi.org/10.11646/zootaxa.2612.1.2

Ehemann NR, González-González LV, Chollet-Villalpando JG, Cruz-Agüero JDL (2018) Updated checklist of the extant Chondrichthyes within the Exclusive Economic Zone of Mexico. ZooKeys 774: 17-39. https://doi.org/10.3897/zookeys.774.25028

Espinoza M, Díaz E, Angulo A, Hernández S, Clarke TM (2018) Chondrichthyan diversity, conservation status, and management challenges in Costa Rica. Frontiers in Marine Science. https://doi. org/10.3389/fmars.2018.00085

Fowler HW (1941) Contributions to the biology of the Philippine archipelago and adjacent regions. The fishes of the groups Elasmocephalii, Holocephali, Isospondyli, and Ostariophysi obtained by the United States Fisheries Steamer "Albatross" in 1907 to 1910, chiefly in the Philippine islands and adjacent seas. Bulletin United States National Museum 100:1-879.

Fricke R, Mahafina J, Behivoke F, Jaonalison H, Léopold M, Ponton D (2018) Annotated checklist of the fishes of Madagascar, southwestern Indian Ocean, with 158 new records. Fishtaxa 3(1): 1-432.

Garrick JAF (1960) Studies on New Zealand Elasmobranchii. Part XII. The species of Squalus from New Zealand and Australia; and a general account and key to the New Zealand Squaloidea. Transactions of the Royal Society of New Zealand 88(3): 519-557.

Hacohen-Domené A, Polanco-Vásquez F, Estupiñan-Montaño C, Graham RT (2020) Description and characterization of the artisanal elasmobranch fishery on Guatemala's Caribbean coast. PLoS ONE 15(1):e0227797. https://doi.org/10.1371/journal.pone.0227797

Herman J, Hovestadt-Euler M, Hovestadt DC (1989) Contributions to the study of the comparative morphology of teeth and other relevant ichthyodorulites in living supraspecific taxa of Chondrichthyes fishes. Part A: Selachii. N ${ }^{\circ}$ 3: Order Squaliformes - Families Echinorhinidae, Oxynotidae and Squalidae. Bulletin de 1'Institut Royal des Sciences Naturelles de Belgique Biologie 59(1):101-157.

IUCN (2020) The IUCN Red List of Threatened Species. Version 20193. http://www.iucnredlist.org

Ishikawa C (1908) Description of a new species of squaloid shark from Japan. Proceedings of the Academy of Natural Sciences of Philadelphia 60: 71-73.

Jones BC, Geen GH (1976) Taxonomic reevaluation of the Spiny Dogfish (Squalus acanthias L.) in the northeastern Pacific Ocean. Journal of the Fisheries Research Board of Canada 33(11): 2500-2506. https://doi.org/10.1139/f76-295

Jordan DS, Snyder JO (1901) A preliminary check list of the fishes of Japan. Annotationes Zoologicae Japonenses 3: 31-159.

Jordan DS, Snyder JO (1903) Squalus. In: Jordan DS, Fowler HW (Eds) A review of the Elasmobranchiate fishes of Japan. Proceedings of the United States National Museum 26(1324): 593-674. https://doi. org/10.5479/si.00963801.26-1324.593

Last PR, Edmunds M, Yearsley GK (2007a) Part 2 - Squalus crassispinus sp nov., a new spurdog of the megalops-cubensis group from the eastern Indian Ocean. In: Last PR, White WT, Pogonoski JJ (Eds) 
Descriptions of new dogfishes of the genus Squalus (Squaloidea: Squalidae). CSIRO Marine and Atmospheric Research Paper 14, Hobart, 11-22.

Last PR, Marshall LJ, White WT (2007b) Part 8 - Squalus nasutus sp. nov, a new long-snouted spurdog of the $S$. japonicus group from the Indian Ocean. In: Last PR, White WT, Pogonoski JJ (Eds) Descriptions of new dogfishes of the genus Squalus (Squaloidea: Squalidae). CSIRO Marine and Atmospheric Research Paper 14, Hobart, 83-90.

Last PR, Séret B, Pogonoski JJ (2007c) Part 3 - Squalus bucephalus sp. nov., a new short-snout spurdog from New Caledonia. In Last PR, White WT, Pogonoski JJ (eds). Descriptions of new dogfishes of the genus Squalus (Squaloidea: Squalidae). CSIRO Marine and Atmospheric Research Paper 14, Hobart, 23-29.

Last PR, Stevens JD (1994) Sharks and Rays of Australia, $1^{\text {st }}$ edition. CSIRO Publishing, Melbourne. https://doi.org/10.2307/1446735

Last PR, White WT, Motomura H (2007d) Part 6 - A description of Squalus chloroculus sp. nov., a new spurdog from southern Australia, and the resurrection of S. montalbani Whitley. In: Last PR, White WT, Pogonoski JJ (Eds) Descriptions of new dogfishes of the genus Squalus (Squaloidea: Squalidae). CSIRO Marine and Atmospheric Research Paper 14, Hobart, 55-69.

Last PR, White WT, Pogonoski JJ (2007e) Descriptions of new dogfishes of the genus Squalus (Squaloidea: Squalidae). CSIRO Marine and Atmospheric Research Paper 14, Hobart.

Last PR, White WT, Pogonoski JJ, Gledhill DC, Yearsleay GK, Ward RD (2007f) Part 1 -Application of a rapid taxonomic approach to the genus Squalus In Last PR, White WT, Pogonoski JJ (eds). Descriptions of new dogfishes of the genus Squalus (Squaloidea: Squalidae). CSIRO Marine and Atmospheric Research Paper 14, Hobart, 1-10.

Last PR, White WT, Stevens J (2007g) Part 5 - New species of Squalus of the highfin megalops group from the Australasian region. In: Last PR, White WT, Pogonoski JJ (Eds) Descriptions of new dogfishes of the genus Squalus (Squaloidea: Squalidae). CSIRO Marine and Atmospheric Research Paper 14, Hobart, 39-53.

Lee WJ, Kim SY, Kim JK (2016) Change of the scientific name for piked dogfish in Korea to Squalus suckleyi (Squalidae, Chondrichthyes) based on morphological and molecular comparisons of Squalus acanthius from New Zealand. Korean Journal of Fisheries and Aquatic Science 49(4): 493-498. https://doi.org/10.5657/ KFAS.2016.0493

Lindberg GU, Legeza MI (1956) Two forms of spiny dogfish Squalus acanthias L. Zool. Zhurn., 35(11) 1685-1688. In: Fishes of the Sea of Japan and adjacent parts of Okhotsk and Yellow Sea. Part 1. Opredeliteli Faune SSSR No. 68: 1-208. [In Russian]

Manjaji-Matsumoto BM (2017) Chondrichthyes. In: Motomura H, Alama UB, Muto N, Babaran RP, Ishikawa S (Eds) Commercial and bycatch market fishes of Panay Island, Republic of the Philippines. The Kagoshima University Museum, Kagoshima, University of the Philippines Visayas, Iloilo, and Research Institute for Humanity and Nature, Kyoto, 19-26.

Matsunaga H, Nakano H, Ishibashi Y, Nakayama K (2003) Estimation of the amount of shark landing by species in the main fishing ports of Japan. The Japanese Society of Fisheries Science 69(2): 178-184. https://doi.org/10.2331/suisan.69.178

McKinnell S, Seki MP (1998) Shark bycatch in the Japanese high seas squid driftnet fishery in the North Pacific Ocean. Fisheries Science 39: 127-138. https://doi.org/10.1016/S0165-7836(98)00179-9
Mejía-Falla PA, Navia AF (2019) Checklist of marine elasmobranchs of Colombia, Universitas Scientiarum 24(1): 241-276. https://doi. org/10.11144/Javeriana.SC24-1.come

Motomura H, Habano A, Arita Y, Matsuoka M, Furuta K, Koeda K, Yoshida T, Hibino Y, Jeong B, Tashiro S, Hata H, Fukui Y, Eguchi K, Inaba T, Uejo T, Yoshiura A, Ando Y, Haraguchi Y, Senou H, Kuriiwa K (2015) The ichthyofauna of the Uji Islands, East China Sea: 148 new records of fishes with notes on biogeographical implications. Memoirs of Faculty of Fisheries Kagoshima University 64: 10-34.

Muñoz-Chápuli R, Ramos F (1989) Morphological comparisons of Squalus blainvillei and S. megalops in the Eastern Atlantic, with notes on the genus. Japanese Journal of Ichthyology 36(1): 6-21. https://doi.org/10.1007/BF02905668

Nakabo T (2002) Fishes of Japan with pictorial keys to the species, English edition. Tokai University Press, Tokyo.

Nakabo T (2013) Fishes of Japan with pictorial keys to the species. Tokai University Press, Tokyo.

Nakano H, Nagasawa K (1996) Distribution of pelagic elasmobranchs caught by salmon research gillnets in the North Pacific. Fisheries Science 62(6): 860-865. https://doi.org/10.2331/fishsci.62.860

Nakaya K (1985) Sharks, Skates, Rays and Chimaeras Taken from Continental Slopes around Japan. Report of Japanese Society for Elasmobranch Studies 19: 1-8.

Nakaya K, Shirai S (1992) Fauna and zoogeography of deep-benthic chondrichthyan fishes around the Japanese Archipelago. Japanese Journal of Ichthyology 39(1): 37-48. https://doi.org/10.1007/BF02905632

Naylor GJP, Caira JN, Jensen K, Rosana AM, White WT, Last PR (2012) A DNA sequence-based approach to the identification of shark and rays species and its implication of global elasmobranch diversity and parasitology. Bulletin of the American Museum of Natural History 367:1-262. https://doi.org/10.1206/754.1

Nión H, Rios C, Meneses P (2016) Peces del Uruguay: Lista sistemática y nombres comunes. Segunda edición corregida y ampliada. DINARA, Montevideo.

Orlov AM, Kulish EF, Mukhametov IN, Shubin OA (2011) Age and Growth of Spiny Dogfish Squalus acanthias (Squalidae, Chondrichthyes) in Pacific Waters off the Kuril Islands. Journal of Ichthyology 51(1): 42-55. https://doi.org/10.1134/S0032945210061049

Orlov AM, Savinykh VF, Kulish EF, Pelenev DV (2012) New data on the distribution and size composition of the North Pacific spiny dogfish Squalus suckleyi (Girard, 1854). Scientia Marina 76(1): 111122. https://doi.org/10.3989/scimar.03439.22C

Parin N (1987) Species of spiny dogfish of genus Squalus, living on Southeastern Pacific Ocean Seamounts. Journal of Ichthyology 27(5): 43-50.

Pfleger MO, Grubbs RD, Cotton CF, Daly-Engel TS (2018) Squalus clarkae sp. nov., a new dogfish shark from the Northwest Atlantic and Gulf of Mexico, with comments on the Squalus mitsukurii species complex. Zootaxa 4444: 101-119. https://doi.org/10.11646/ zootaxa.4444.2.1

Quang VV, Tuan VS, Thao LTT, Thinh TC, Uy Vu NP, Phuong LM (2013) Species composition and distribution of sharks in Quy Nhon Bay and neighboring waters. Vietnam Journal of Marine Science and Technology 13(1): 21-30. https://doi.org/10.15625/1859_ 3097/13/1/3503

Quantum GIS Development Team (2018) Quantum GIS Geographic Information System, Open Source Geospatial Foundation Project; http://qgis.osgeo.org/ 
Sabaj MH (2016) Standard symbolic codes for institutional resource collections in herpetology and ichthyology: an Online Reference. American Society of Ichthyologists and Herpetologists, Washington DC., electronic version 6.5, updated 16 August 2016. http://www.asih.org/

Shinohara G, Nakae M, Ueda Y, Kojima S, Matsuura K (2014) Annotated checklist of deep-sea fishes of the Sea of Japan. In: Fujita T (Ed.) Deep-sea fauna of the Sea of Japan. National Museum of Nature and Science Monographs 44, Tokyo, 225-291.

Shirai S (1989) A key to the species of the dogfishes (Squaloidei) from Japanese waters. Report of Japanese Society for Elasmobranch Studies 26: 1-35.

Siming D, Guoqiang X, Hongxi Z (1988) Chondrichthyes. In: Jinao S, Yuanquan C (Eds) A general environmental feature of the deep water of East China Sea. Xue Lin Publishing House, Shangai, 1 pp.

Sonu SC (1998) Shark fisheries, trade, and market of Japan. NOAA Technical Memorandum NMFS 033: 1-70.

Springer VG, Garrick JAF (1964) A survey of vertebral numbers in sharks. Proceedings of the United States Natural Museum 116(3496): 73-96. https://doi.org/10.5479/si.00963801.116-3496.73

Straube N, White WT, HO H-C, Rochel E, Corrigan S, Li C, Naylor GJP (2013) A DNA sequence-based identification checklist for Taiwanese chondrichthyans. Zootaxa 3752: 256-278. https://doi. org/10.11646/zootaxa.3752.1.16

Tanaka S (1917) Figures and descriptions of the fishes of Japan, including the Riukiu Islands, Bonin Islands, Formosa, Kurile Islands, Korea, and southern Sakhalin. Figures and Descriptions of the Fishes of Japan 26: 455-474.

Vella A, Vella N, Schembri S (2017) A molecular approach towards taxonomic identification of elasmobranch species from Maltese fisheries landings. Marine Genomics 36: 17-23. https://doi.org/10.1016/j. margen.2017.08.008

Veríssimo A, McDowell JR, Graves JE (2010) Global population structure of the spiny dogfish Squalus acanthias, a temperate shark with an antitropical distribution. Molecular Ecology 19: 1651-1662. https://doi.org/10.1111/j.1365-294X.2010.04598.x

Veríssimo A, Zaera-Perez D, Leslie R, Iglésias SP, Séret B, Grigoriou P, Sterioti A, Gubili C, Barría C, Duffy C, Hernández S, Batjakas IE, Griffiths AM (2017) Molecular diversity and distribution of eastern Atlantic and Mediterranean dogfishes Squalus highlight taxonomic issues in the genus. Zoologica Scripta 46(4): 414-428. https://doi. org/10.1111/zsc. 12224

Viana STF, Carvalho MR (2016) Redescription of Squalus acutipinnis Regan, 1908, a valid species of spiny dogfish from Southern Africa (Chondrichthyes: Squaliformes: Squalidae). Copeia 104(2): 539553. https://doi.org/10.1643/CI-14-217

Viana STFL, Carvalho MR (2018a) Squalus rancureli, a new junior synonym of the blacktailed spurdog S. melanurus, and updated diagnosis of $S$. bucephalus from New Caledonia (Chondrichthyes: Squaliformes: Squalidae). Zoosystema 40(9): 159-177. https://doi. org/10.5252/zoosystema2018v40a9

Viana STFL, Carvalho MR (2018b) Resurrection and redescription of the Southern dogfish Squalus probatovi (Squalidae), a valid species from Angola. Journal of Ichthyology 58(5): 617-632. https://doi. org/10.1134/S003294521805020X

Viana STF, Carvalho MR, Gomes UL (2016) Taxonomy and morphology of species of the genus Squalus Linnaeus, 1758 from the Southwestern Atlantic Ocean (Chondrichthyes: Squaliformes: Squalidae). Zootaxa 4133(1): 1-89. https://doi.org/10.11646/zootaxa.4133.1.1
Viana STFL, Lisher MW, Carvalho MR (2017a) Two new species of short-snouted dogfish sharks of the genus Squalus Linnaeus, 1758 from Southern Africa (Chondrichthyes: Squaliformes: Squalidae). Marine Biodiversity 48(8): 1787-1814. https://doi.org/10.1007/s12526-0170673-8

Viana STFL, Carvalho MR, Ebert DA (2017b) Squalus bassi sp. nov., a new long-snouted spurdog (Chondrichthyes: Squaliformes: Squalidae) from the Agulhas Bank. Journal of Fish Biology 91(4): 11781207. https://doi.org/10.1111/jfb.13448

Ward RD, Holmes BH, Zemlak TS, Smith PJ (2007) DNA barcoding discriminates spurdogs of the genus Squalus. In: Last PR, White WT, Pogonoski JJ (Eds) Descriptions of new dogfishes of the genus Squalus (Squaloidea: Squalidae). CSIRO Marine and Atmospheric Research Paper 14, Hobart, 117-130.

Weigmann S (2016) Annotated checklist of the living sharks, batoids and chimaeras (Chondrichthyes) of the world, with a focus on biogeographical diversity. Journal of Fish Biology 88(3): 837-1037. https://doi.org/10.1111/jfb.12874

White WT, Iglésias SP (2011) Squalus formosus, a new species of spurdog shark (Squaliformes: Squalidae), from the Western North Pacific Ocean. Journal of Fish Biology 79(4):1-15. https://doi. org/10.1111/j.1095-8649.2011.03068.x

White WT, Last PR (2013) Notes on shark and ray types at the South China Sea Fisheries Research Institute (SCSFRI) in Guangzhou, China. Zootaxa 3752: 228-248. https://doi.org/10.11646/zootaxa.3752.1.14 White WT, Last PR, Stevens JD (2007a) Part 7 - Two new species of Squalus of the mitsukurii group from the Indo-Pacific. In: Last PR, White WT, Pogonoski JJ (Eds) Descriptions of new dogfishes of the genus Squalus (Squaloidea: Squalidae). CSIRO Marine and Atmospheric Research Paper 14, Hobart, 71-81.

White WT, Last PR, Yearsley GK (2007b) Part 10 - Squalus hemipinnis sp. nov, a new short-snouted spurdog from eastern Indonesia. In: Last PR, White WT, Pogonoski JJ (Eds) Descriptions of new dogfishes of the genus Squalus (Squaloidea: Squalidae). CSIRO Marine and Atmospheric Research Paper 14, Hobart, 101-108.

White WT, Yearsley GK, Last PR (2007c) Part 11 - Clarification of the status of Squalus tasmaniensis and a diagnosis of Squalus acanthias from Australia, including a key to the Indo-Australasian species of Squalus. In: Last PR, White WT, Pogonoski JJ (Eds) Descriptions of new dogfishes of the genus Squalus (Squaloidea: Squalidae). CSIRO Marine and Atmospheric Research Paper 14, Hobart, 109-115.

Wilson CD, Seki MP (1994) Biology and population characteristics of Squalus mitsukurii from a seamount in the central North Pacific Ocean. Fisheries Bulletin 92: 851-864.

Yano T, Ohshimo S, Kanaiwa M, Hattori T, Fukuwaka Ma, Nagasawa T, Tanaka S (2017) Spatial distribution analysis of the North Pacific spiny dogfish, Squalus suckleyi, in the North Pacific using generalized additive models. Fisheries Oceanography 26: 668-679. https:// doi.org/10.1111/fog.12225

Yamashita M, Yoshida T, Motomura H (2012) An illustrated list of the Kagoshima University Museum specimens of Chondrichthyes collected from Kagoshima Prefecture, southern Japan. Nature of Kagoshima 38: 119-138.

Zhu Y (1960) Classification of Chondrichthyes of China. Science Press, Beijing, China.

Zhu Y, Meng QW, Li S (1984) Description of a new species of Squalidae of China. Oceanologia et Limnologia Sinica 15(4): 283-286.

Zhu Y, Meng QW (2001) Faina Sinica - Cyclostomata, Chondrichthyes. Science Press, Beijing. 


\section{Supplementary material 1}

\section{Table S1}

Authors: Sarah T. F. L. Viana, Marcelo R. de Carvalho Data type: vertebral counts

Explanation note: Range values of vertebral counts for species of Squalus are summarised for comparisons.

Copyright notice: This dataset is made available under the Open Database License (http://opendatacommons. org/licenses/odbl/1.0/). The Open Database License $(\mathrm{ODbL})$ is a license agreement intended to allow users to freely share, modify, and use this Dataset while maintaining this same freedom for others, provided that the original source and author(s) are credited.

Link: https://doi.org/10.3897/zse.96.51962.suppl1 